\title{
Prediction of the Favorable Hydration Sites in a Protein Binding Pocket and Its Potential Application to Scoring Function Formulation
}

Yan Li, ${ }^{a *}$ Yingduo Gao, ${ }^{b}$ M. Katharine Holloway, ${ }^{c}$ and Renxiao Wang ${ }^{a, d, e *}$

${ }^{a}$ Department of Medicinal Chemistry, School of Pharmacy, Fudan University, 826 Zhangheng Road, Shanghai 201203, People's Republic of China

${ }^{b}$ Merck Research Laboratories, 2000 Galloping Hill Road, Kenilworth, New Jersey 07033, and 770 Sumneytown Pike, West Point, Pennsylvania 19486, U. S. A.

${ }^{c}$ Gfree Bio LLC, 3805 Old Easton Road, Doylestown, Pennsylvania 18902, U. S. A.

${ }^{d}$ State Key Laboratory of Bioorganic and Natural Products Chemistry, Center for Excellence in Molecular Synthesis, Shanghai Institute of Organic Chemistry, Chinese Academy of Sciences, 345 Lingling Road, Shanghai 200032, People's Republic of China

${ }^{e}$ Shanxi Key Laboratory of Innovative Drugs for the Treatment of Serious Diseases Basing on Chronic Inflammation, College of Traditional Chinese Medicines, Shanxi University of Chinese Medicine, Taiyuan, Shanxi 030619, People’s Republic of China

\section{TABLE OF CONTENTS}

PART 1. Additional details and results of the HydraMap method

PART 2. Basic information of the data sets used for calibrating and validating the derivative scoring functions 18

PART 3. Integration of desolvation energies by water displacement into scoring functions ... 26 PART 4. Comparisons of the performance of original scoring functions and their derivative scoring functions with additional desolvation terms 30 


\section{PART 1. Additional details and results of the HydraMap method}

\section{Mean shift clustering algorithm in HydraMap}

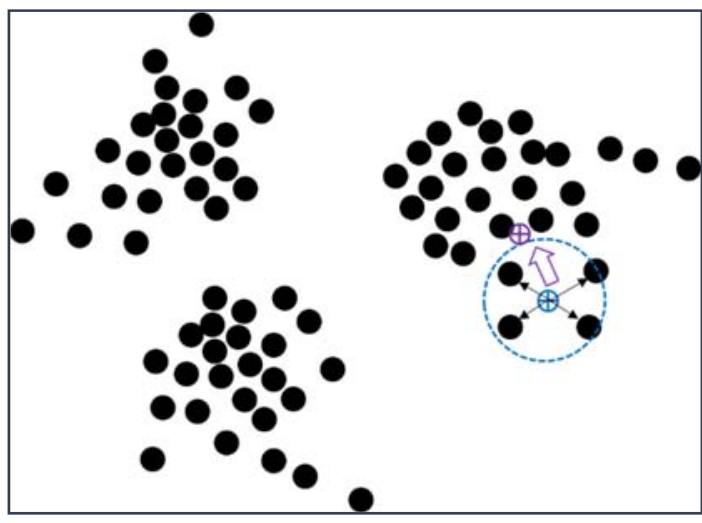

(a)

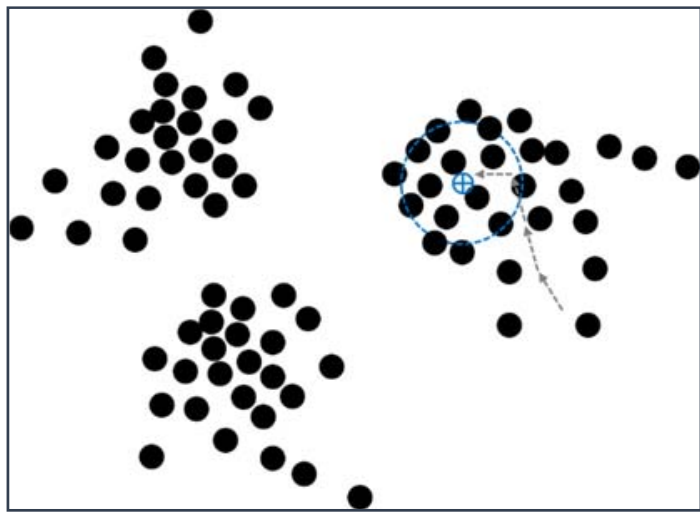

(c)

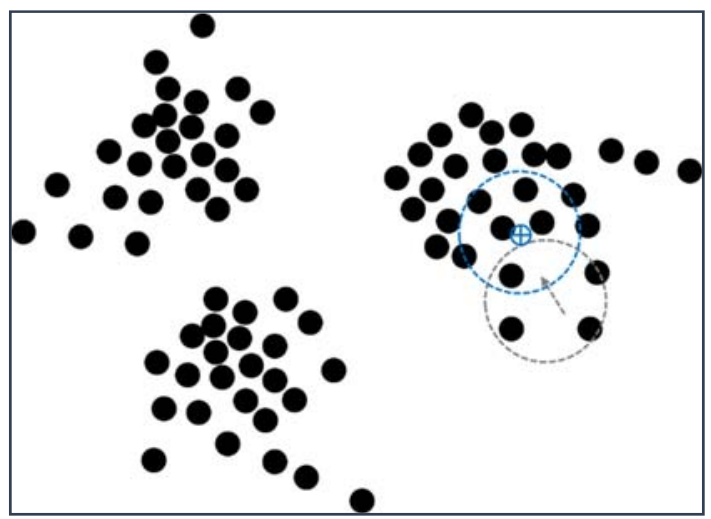

(b)

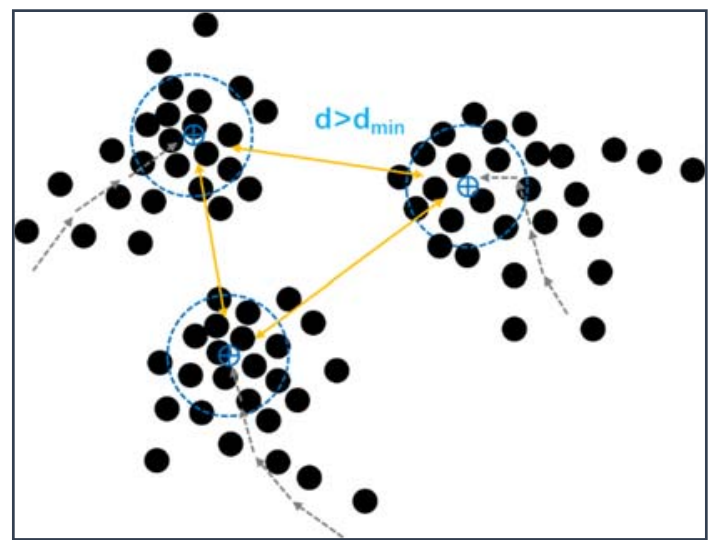

(d)

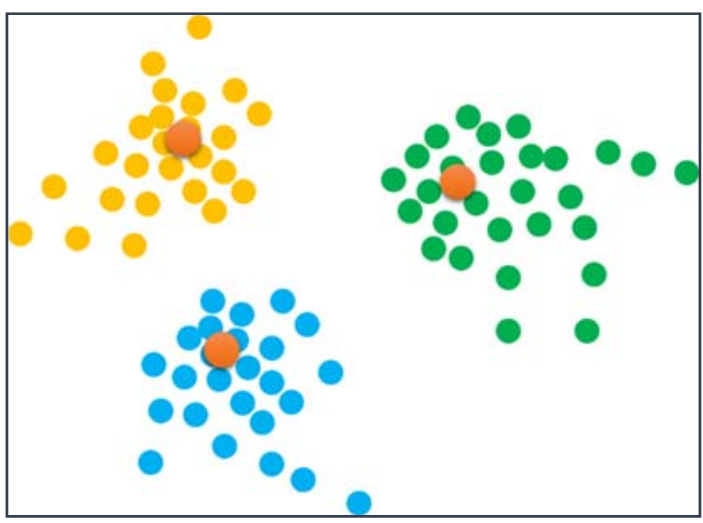

(e)

Figure S1. Illustration of the Mean Shift algorithm in clustering. (a) Select a center point randomly and calculate the average vector shift from the grids within the region defined by the center point. (b) Move the center along the direction of the shift, and calculate the average vector shift from the grids within the region defined by the new center. (c) Repeat the previous step until the center does not move any more. All relevant 
grids are grouped into the cluster surrounding this center. (d) Start from another center point and do steps (a)(c). If the centers of two clusters are within the defined distance cutoff, merge them into one cluster; else they are two separate clusters. (e) Final clusters are determined.

\section{Parameter optimization of HydraMap}

There are four key parameters in our HydraMap method, including the distance interval of grids (grid_spacing), the dimension length of the grid box $(\boldsymbol{D})$, the defined energy cutoff for favorable hydration site $(\boldsymbol{E})$ and the minimal size of a cluster to define a valid hydration site $(\boldsymbol{N})$. We optimized these parameters on the "HQ_Set" (Table S1), which was compiled as described in the manuscript. The final values of these parameters used in our study were adjusted manually through a stepwise procedure as follows.

Firstly, two values for grid_spacing were defined (i.e. $0.50 \AA$ and $0.25 \AA$ ), with two sets of the other three parameters (set-1: $\boldsymbol{D}=3.0, \boldsymbol{E}=5.0, \boldsymbol{N}=30$; set-2: $\boldsymbol{D}=4.0, \boldsymbol{E}=5.0, \boldsymbol{N}=30$ ). For both sets, when the grid_spacing value was $0.25 \AA$, the prediction success rates were marginally higher than those obtained when grid_spacing $=0.50 \AA$. The improvement was about $1.7 \%-3.5 \%$ when the prediction hydration site was considered to reproduce the crystal water if their distance was shortened than 1.0 $\AA$ (level-I), $1.5 \AA$ (level-II) and $2.0 \AA$ (level-III) (Figure S2(a)). However, the computation time increased by $2-3$ times as a consequence. Thus, grid_spacing $=0.50 \AA$ is recommended for a real high-throughput task. In this study, however, the grid_spacing value was set to $0.25 \AA$ in a hope to achieve a higher level of accuracy.

Next, the dimension length of the grid box $(\boldsymbol{D})$ was varied as $3.0 \AA, 4.0 \AA$ and $5.0 \AA$ while the other parameters were fixed (i.e.grid_spacing $=0.25, \boldsymbol{E}=5.0, \boldsymbol{N}=30$ ). The best success rate was obtained when $\boldsymbol{D}=4.0 \AA$, as shown in Figure S2(b). The improvement was about $2.7 \%$, $4.9 \%$ and $5 \%$ at three levels. Next, the grid number in a cluster to represent a valid hydration site $(\boldsymbol{N})$ was varied as $10,20,30$ and 40 while the other parameters were fixed (i.e. grid_spacing $=0.25, \boldsymbol{D}=4.0, \boldsymbol{E}=5.0$ ). The optimal value of $\boldsymbol{N}$ was set as 10 due to the highest success rate obtained at level-II (Figure S2(c)). Finally, the energy cutoff for favorable hydration site $(\boldsymbol{E})$ was varied as $0.0,1.0,2.0,3.0,4.0$ and 5.0 while the other parameters fixed (i.e. grid_spacing $=0.25, \boldsymbol{D}=4.0, \boldsymbol{N}=10$ ). At all three levels, the highest success rates were obtained when $\boldsymbol{E}=5.0$, which was then taken as the optimal value (Figure S2(d)).

It was too time-consuming to derive the optimal setting of these parameters by exploring all possibilities systematically on the entire HQ_Set. We hope that the setting of these 
parameters described above is acceptable and not too far from the optimal one.

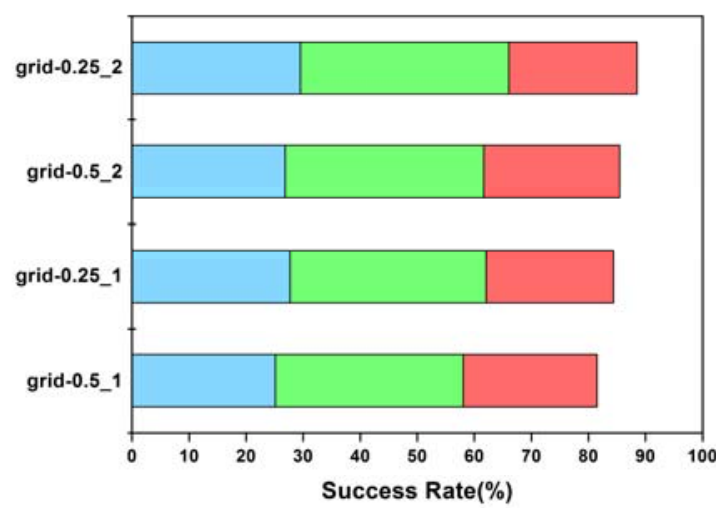

(a)

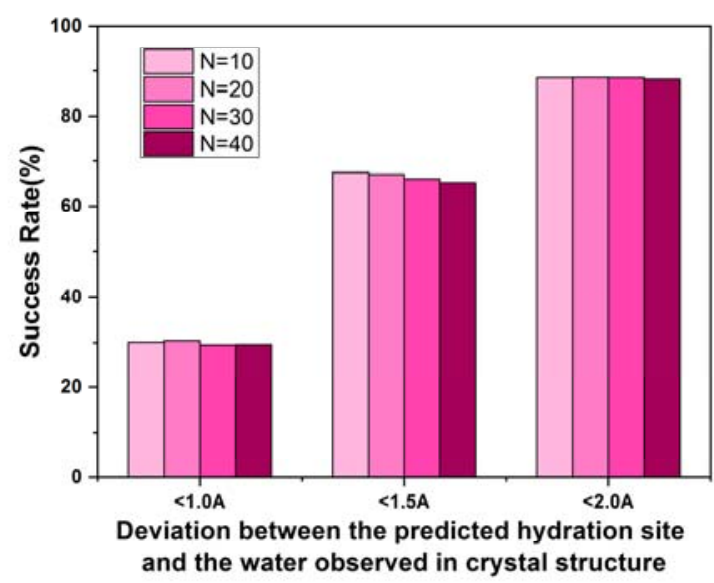

(c)

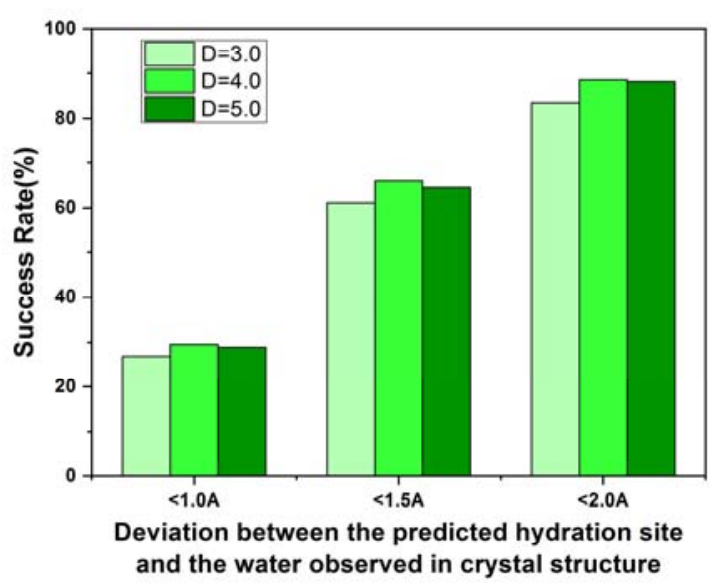

(b)

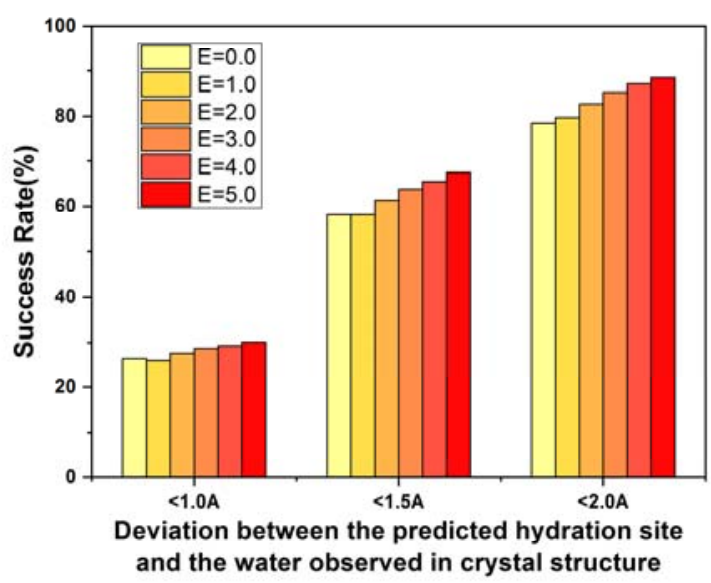

(d)

Figure S2. Hydration site prediction success rates of our method HydraMap under different parameter settings. (a) grid_spacing values were $0.25 \AA$ and $0.5 \AA$ with two settings (set-1: $\boldsymbol{D}=3.0, \boldsymbol{E}=5.0, N=30$; set-2: $\boldsymbol{D}=4.0, \boldsymbol{E}=5.0, \boldsymbol{N}=30$ ). The deviation between the predicted hydration site and the water observed in crystal structure was less than $1.0 \AA$ (light blue), $1.5 \AA$ (light green) and $2.0 \AA$ (light red). (b) the dimension length of the grid box $\boldsymbol{D}$ was set as $3.0 \AA$ (light green), $4.0 \AA$ (green) and $5.0 \AA$ (dark green). (c) the grid number $(N)$ in a cluster for a hydration site was set as 10 (light pink), 20 (pink), 30 (dark pink) and 40 (dark rose red). (d) the energy cutoff $(\boldsymbol{E})$ was set as 0.0 (light yellow), 1.0 (yellow), 2.0 (light orange), 3.0 (orange), 4.0 (light red) and 5.0 (red). 
Table S1. List of the protein-ligand complexes in the high-quality data set for optimizing parameters in hydration site prediction algorithm 1C1P 1C1Q 1C1R 1C1T 1C2H 1C2I 1C2J 1C5Q 1C5R 1EK0 1F4P 1F8D 1F8E 1F9V 1FLM 1G67 1G6C 1GA4 1GHZ 1GI0 1GI1 1GI2 1GI3 1GI4 1GI6 1GK8 1GM7 1GVO 1GX4 1GYX 1GZ8 1H10 1H11 1H2J 1H4G 1H61 1HET 1HEU 1HF6 1HHU 1HQ2 1I0V 1I24 1I5G 1I73 1I76 1IE9 1IW0 1J3F 1J96 1J9B 1JBO 1JCJ 1JG1 1JUB 1KA1 1KB0 1KJQ 1KQR 1KY3 1KYP 1KYS 1KZK 1L9L 1LJU 1LLF 1LLR 1LQT 1LQU 1LRI 1LZI 1M22 1M5E 1M7G 1MD2 1MGO 1MPL 1N8K 1N92 1NEY 1NLU 1NNF $1 \mathrm{O} 041 \mathrm{O} 08$ 1O0H 1O0O 1O2P 1O2R 1O2U 1O2W 1O2X 1O2Y $1 \mathrm{O} 331 \mathrm{O} 351 \mathrm{O} 37$ 1O38 1O3D 1O3J 1O3K 1O3L 1O8B 1OBD 1OBN 1OBO 1OC7 1OCJ 1OD1 1ODM 1OEX 1OH0 1OI6 1ONH 1OS6 1P4C 1P5B 1PI4 1PI5 1PW8 1PWC 1PYZ 1PZ4 1PZP 1Q0N 1Q0R 1Q2Q 1Q4A 1Q4B 1Q4E 1Q5M 1Q6O 1Q92 1QJF 1QOP 1QW9 1QWN 1QXY 1QYZ 1QZ5 1R50 1R6D 1R8S 1RB0 1RDQ 1RE9 1RGZ 1RL9 1RMT 1RMZ 1RU1 1RUM 1RWF 1SCW 1SDE 1SDT 1SDU 1SDV 1SEH 1SL9 1SQN 1SR7 1T2D 1T5B 1T7R 1T8Z 1TF9 1TKY 1TQS 1TQW 1TZC 1U3W 1U4G 1U7G 1UAY 1UB3 1URS 1USC 1USF 1UUY 1UWK 1UZW 1VE1 1VEF 1VMF 1VP8 1VYP 1W04 1W3V 1W3X 1W6S 1WB4 1WB5 1WBI 1WDD 1WDQ 1WDR 1WMA 1WQW 1WUA 1WVF 1WVQ 1WZD 1X1R 1X3S 1X54 1X96 1X97 1X98 1X9D 1X9I 1XBI 1XBU 1XDN 1Y2K 1Y55 1Y5A 1YBK 1YLJ 1YLP 1YLT 1YLY 1YLZ 1YMT 1YNO 1YNP 1YNQ 1Z0J 1Z2N 1Z2O 1Z2P 1Z41 1Z53 1Z70 1ZDY 1ZGO 1ZK4 1ZKK 1ZQ5 1ZUA 2A26 2A2R 2A3U 2A49 2A50 2A53 2A54 2A8Y 2ABA 2ABB 2AGT 2AGW 2AW1 2AWK 2AYW 2B69 2B82 2BCG 2BF6 2BFD 2BFF 2BHZ 2BIE 2BK9 2BKX 2BU3 2BU9 2C01 2C04 2C0C 2C0R 2C15 2C3V 2C4J 2C5A 2C78 2C9J 2CC6 2CF8 2CIA 2CN0 2CNQ 2CVD 2CXV 2CZ2 2D1S 2D1T 2D5M 2D5X 2D5Z 2DJL 2DKJ 2DT8 2DUG 2DUH 2DUI 2DXU 2E3N 2E3P 2E68 2E6F 2E7Z 2ECU 2EGV 2EIY 2EKP 2F01 2F18 2F1A 2F1B 2F5T 2F5V 2F69 2F7P 2F7R 2F80 2FFY 2FGB 2FGQ 2FLH 2FN3 2FOQ 2FR5 2FWN 2FWQ 2FXU 2FZ8 2FZU 2G5V 2G6E 2G8T 2GCO 2GD8 2GEW 2GF3 2GG0 2GG2 2GH7 2GOU 2GQT 2GQW 2GW3 2GXQ 2H4E 2H5P 2H5Q 2H5S 2H6M 2H6V 2H8Z 2H90 2H9H 2HAL 2HB3 2HCG 2HEJ 2HJO 2HOX 2HQK 2HQZ 2HRS 2HU6 2HUB 2HXC 2HXM 2HXS 2HYV 2HZY 2I0R 2I51 2I7D 2I7S 2IGC 2IKI 2IMF 2IMI 2ITK 2IUR 2IVI 2IVJ 2IYV 2J6L 2J9K 2JAE 2JB2 2JB4 2JG2 2JKH 2NMY 2NN8 2NNG 2NNK 2NNS 2NUI 2O0B $2 \mathrm{O} 232 \mathrm{O} 24$ 2O90 2O9C 2OF8 2OK6 2ORD 2OSX 2OTE 2OUR 2OVJ 2OXC 2P02 2P4T 2P86 2PDH 2PDL 2PFE 2PGN 2PGO 2PHN 2PIY 2PR5 2PY0 2PY4 2Q0L 2Q0U 2Q7W 2QAA 2QB3 2QCF 2QCI 2QD6 2QD7 2QGE 2QNN 2QNT 2QO8 2QOC 2QP6 2QT2 2QUZ 2QXI 2QXW 2QZ0 2R01 2R14 2R3I 2R3Q 2R3R 2R75 2R8O 2RCT 2RDN 2RFV 2RGB 2RGE 2RGG 2RJ4 2RJ5 2RJ6 2RK1 2RL8 2UV4 2UVO 2UX9 2UXW 2V03 2V0U 2V52 2V6K 2VBA 2VBB 2VCH 2VD8 2VEZ 2VFR 2VIF 2VK7 2VK8 2VLW 2VOV 2VUC 2VXV 2W12 2W13 2W14 2W15 2W1P 2W1S 2W47 2W4J 2W9H 2WCJ 2WD2 2WD4 2WDS 2WDY 2WE5 2WF5 2WF6 2WF7 2WF8 2WF9 2WK1 2WKJ 2WLR 2WQF 2WSB 2WSN 2WSO 2WUR 2WZB 2WZX 2X18 2X2O 2X2P 2X5O 2X6W 2X7K 2X9G 2X9V 2XDE 2XFF 2XGB 2XGI 2XN6 2XN7 2XNQ 2XOE 2XPC 2XPV 2XPW 2XQO 2XRW 2XU1 2XU3 2XWL 2XWV 2XZC 2XZI 2Y1O 2Y53 2Y5D 2Y60 2Y66 2Y68 2Y8E 2YC3 2YCD 2YEX 2YGJ 2YHG 2YI7 2YK9 2YKE 2YKJ 2YVE 2Z6W 2Z7K 2Z98 2ZD8 2ZFF 2ZL7 2ZO6 2ZPT 2ZQ9 2ZQA 2ZQC 2ZQD 2ZSC 2ZVP 2ZVQ 2ZWS 3A35 3A40 
3A6Q 3A6R 3A8U 3A9B 3ABX 3AGN 3AGO 3AHW 3AIA 3AJ6 3ALJ 3ARX 3ARY 3AU2 3AZ3 3B0T 3B0X 3B0Y 3B28 3B3R 3B50 3B6D 3B7E 3B7V 3B8Z 3B9G 3BBB 3BC9 3BER 3BHL 3BJE 3BKR 3BLL 3BOG 3BUV 3BUW 3BUX 3BVA 3BVB 3BVT 3BVV 3BWY 3C31 3C3Y 3C9U 3CB9 3CBE 3CBP 3CD1 3CI3 3CIW 3CNJ 3CV2 3CYW 3CYX 3CZ9 3D1X 3D20 3D3B 3D80 3DBK 3DCR 3DD0 3DHA 3DHB 3DHC 3DKE 3DLC 3DOU 3DQ4 3DQ7 3DQ9 3DQA 3DQC 3DQD 3DQE 3DQF 3DQH 3DQI 3DQK 3DQL 3DQM 3DQN 3DQU 3DT4 3DTB 3DUW 3DYB 3E10 3E4V 3E8T 3EDO 3EIN 3EJP 3EJQ 3EJR 3EJS 3EJT 3EJU 3ELF 3EPW 3ESC 3ESN 3ESO 3ESP 3EVF 3EVP 3EWW 3EWX 3EWY 3EWZ 3EX1 3EX3 3EX4 3EX6 3F03 3F0L 3F16 3F17 3F18 3F19 3F1A 3F5L 3F66 3F6B 3F6E 3F8D 3F8Y 3F9B 3F9X 3FCI 3FE7 3FEA 3FEC 3FED 3FO7 3FRH 3FS6 3FSJ 3FUC 3FVP 3FW9 3FZW 3G1V 3G32 3G34 3G35 3G3M 3G5H 3G5S 3G6K 3GAH 3GAI 3GAJ 3GBA 3GC5 3GFA 3GHC 3GHV 3GHW 3GIX 3GJ0 3GKN 3GR3 3GU4 3GU6 3GVL 3GYI 3GYJ 3H1X 3H4I 3H4T 3H5B 3H62 3H63 3H6W 3H7R 3HAU 3HAW 3HD1 3HD2 3HJ0 3HLX 3HP4 3HSG 3HT0 3HV8 3HVH 3HVI 3HVK 3HYG 3HZA 3HZC 3I06 3I0G 3I0J 3I19 3I1U 3I33 3I3S 3I4G 3I6C 3I6O 3I8U 3I95 3IA9 3IB0 3IBO 3IBU 3ICV 3IIS 3IIU 3IIX 3IJ8 3IJJ 3ILO 3IMS 3IOF 3IOG 3IOI 3IP0 3IPE 3IQV 3IR4 3ISG 3IU7 3IUT 3IX3 3JWA 3JZJ 3K5X 3K82 3K8Y 3KA2 3KFA 3KI0 3KI1 3KI2 3KI3 3KI7 3KKP 3KKQ 3KKU 3KL6 3KPE 3KUH 3KUS 3KWZ 3L0F 3L1N 3L5L 3L5M 3L65 3L66 3L8Z 3LA1 3LBO 3LD5 3LEZ 3LF3 3LFT 3LHS 3LHT 3LHV 3LHW 3LHY 3LHZ 3LI1 3LJG 3LLD 3LLF 3LN3 3LOJ 3LQG 3LQL 3LTP 3LTS 3LV5 3LV6 3LVC 3LZ5 3M07 3M1O 3M1V 3M1Z 3M2R 3M2U 3M30 3M32 3M58 3M64 3M6B 3M6W 3M73 3M96 3MD7 3MEU 3MG5 3MHO 3MHY 3MI2 3MJE 3MJS 3MJV 3MKF 3MO7 3MOE 3MP6 3MQG 3MQH 3MWS 3MXR 3MXS 3MYQ 3N1S 3N3M 3N8L 3N8R 3NB8 3NBD 3NCQ 3NCR 3NED 3NHI 3NHT 3NK8 3NOQ 3NQ7 3NQE 3NQF 3NSZ 3NTZ 3NU0 3NU4 3NU5 3NU6 3NUA 3NUO 3NXO 3NXR 3NXX 3NY4 3NYC 3NYE 3NYF 3NYG 3NYQ 3NYR 3NYS 3NZB 3O0I 3O18 3O1C 3O1X 3O22 3O7B 3O8Q 3O9I 3O9Z 3OCJ 3OCU 3OCZ 3OE4 3OGN 3OGV 3OIG 3OIM 3OIU 3OIW 3OKU 3OKV 3ONB 3OND 3ONE 3OPH 3OQ6 3OT3 3OVX 3OYQ 3OZS 3OZT 3P0K 3P17 3P3C 3P3E 3P3R 3P3T 3P5Z 3P60 3P61 3P62 3P6G 3P73 3P74 3P7Y 3P80 3P81 3P84 3P8G 3P8I 3P9Q 3PA3 3PB7 3PBU 3PBV 3PBW 3PBY 3PBZ 3PC0 3PFG 3PFX 3PFZ 3PIB 3PKA 3PKB 3PKC 3PKD 3PL3 3PL8 3PLD 3PMJ 3PMP 3PMU 3PMY 3PN3 3PO6 3POT 3PPL 3PPT 3PRS 3PSY 3PWR 3PX8 3Q27 3Q3Y 3Q46 3Q4O 3Q6X 3Q6Y 3Q70 3QAA 3QBF 3QDV 3QE8 3QF0 3QFS 3QFT 3QGZ 3QIH 3QIO 3QMR 3QMS 3QMT 3QN8 3QP0 3QPA 3QPC 3QSD 3QVP 3QVR 3QXC 3QXH 3QXJ 3QXS 3QXX 3QYK 3QYZ 3QZR 3R0F 3R3S 3R4G 3R56 3R5T 3R72 3R96 3R9E 3R9F 3R9G 3RCF 3RD9 3RDK 3RDO 3RHB 3RJ5 3RJ7 3RL3 3RL4 3RLU 3RLV 3RM0 3RND 3RS0 3RUN 3RXW 3RYJ 3RYV 3RYY 3RYZ 3S0B 3S0K 3S1Y 3S2J 3S2L 3S2M 3S2N 3S43 3S44 3S54 3S6F 3S74 3S7O 3S8X 3S92 3S9T 3SAX 3SBI 3SF0 3SHL 3SIG 3SII 3SIZ 3SJ3 3SK2 3SPK 3SR1 3SRY 3SS0 3SSH 3SSJ 3SSK 3SSL 3SST 3ST0 3ST5 3SU1 3SU3 3SU6 3SV6 3SVB 3SVC 3SVE 3SX3 3SX5 3SX7 3SX8 3SXB 3SXE 3SXH 3SY0 3SY5 3SZJ 3T10 3T2D 3T42 3T65 3T6F 3T6I 3T74 3T7P 3T7Q 3T7X 3T87 3T8D 3T8F 3T8H 3T8N 3T94 3TB4 3TC5 3TCT 3TEM 3TG2 3TH9 3TKD 3TL9 3TLC 3TLE 3TLL 3TME 3TNL 3TOF 3TOG 3TOH 3TQ5 3TRV 3TV3 3TW2 3TYI 3U2U 3U5L 3U7R 3U81 3U91 3U98 3U9W 3UAW 3UAX 3UAY 3UAZ 3UFN 3UGC 3UHM 3UJ8 3UKO 3UP3 3UVC 3UVK 3UWI 3UXJ 3V1P 3V2M 3V3W 3V4F 3V50 3V5M 3VF3 3VF5 3VF7 3VFA 3VGI 3VHA 3VHC 3VHV 
3VIK 3VIN 3VK5 3VO2 3VOG 3VW9 3VY5 3VYP 3W04 3W0T 3W1A 3W1C 3W1X 3W23 3W2J 3W6Z 3WCZ 3WDC 3WDD 3WDE 3WFG 3WIW 3WJZ 3WK0 3WK3 3ZCG 3ZI4 3ZKU 3ZKY 3ZL2 3ZME 3ZMR 3ZP9 3ZTV 3ZXH 3ZZS 4A6R 4A6V 4A6W 4A7G 4A7Q 4A7S 4A7T 4ABF 4ABJ 4AGO 4AGQ 4AK2 4AR7 4AS8 4ATT 4AV5 4AW0 4AW7 4AWS 4AYO 4AYP 4AYQ 4AZ6 4B1Y 4B4U 4B5N 4B7H 4BAD 4BAP 4BAR 4BB3 4BB9 4BCT 4BEU 4BF1 4BF5 4BGB 4BKD 4BN4 4BRC 4BRG 4BRQ 4BS5 4BW1 4CFL 4D9W 4DDS 4DDY 4DE0 4DE1 4DE2 4DE3 4DFA 4DFG 4DFX 4DGZ 4DHR 4DI9 4DJA 4DJP 4DJQ 4DKN 4DLR 4DQ5 4DQE 4DQJ 4DRM 4DRN 4DRO 4DWR 4DXM 4DZ7 4DZ9 4E2B 4E2X 4E2Z 4E3K 4E3L 4E3M 4E3N 4E3X 4EA7 4EAA 4EAB 4EDE 4EET 4EEU 4EFP 4EGU 4EK4 4EOD 4EPV 4EQP 4ESE 4EU9 4EUL 4EV4 4EY2 4EYB 4F0B 4F3I 4F5L 4F66 4F8Z 4FAT 4FB7 4FCF 4FEE 4FEG 4FGL 4FH2 4FH4 4FI6 4FI8 4FKL 4FKU 4FLK 4FPS 4FPT 4FRA 4FVO 4FYO 4G1X 4G41 4GC4 4GCJ 4GES 4GG1 4GG9 4GH0 4GH1 4GIE 4GKC 4GM0 4GM1 4GM4 4GM5 4GMU 4GNQ 4GNU 4GOG 4GP9 4GPE 4GPQ 4GQQ 4GQR 4GR3 4GR8 4GRR 4GT9 4GV1 4GVF 4GVH 4GVQ 4H48 4H4D 4H4I 4H4R 4H4V 4H8E 4H8R 4HBU 4HCT 4HCU 4HDB 4HDF 4HDP 4HE9 4HEG 4HEQ 4HI7 4HJT 4HL2 4HMJ 4HP8 4HPJ 4HT2 4HT3 4HTG 4HXS 4HY4 4HY7 4HYI 4HZF 4HZM 4I2U 4I3B 4I3G 4I4S 4I71 4I7V 4I7W 4IAV 4ICI 4IDC 4IFX 4IG1 4IGT 4IIX 4IIY 4IK2 4IKU 4INE 4INW 4IPS 4IPW 4IQ9 4IS5 4ITA 4ITB 4J4Z 4J55 4J74 4J7D 4J8A 4JCK 4JD5 4JFJ 4JFK 4JFL 4JFM 4JJE 4JLL 4JMB 4JPM 4JU8 4JV8 4JVO 4JX9 4JXH 4JYF 4JZZ 4K4O 4K7T 4K9K 4KAP 4KB9 4KGD 4KJJ 4KJK 4KJL 4KL9 4KLX 4KM0 4KM2 4KNN 4KOR 4KOW 4KOY 4KP5 4KS7 4KTF 4KTK 4KXU 4KXY 4KY2 4KY5 4KZV 4L1I 4L1T 4L2H 4L6D 4L9P 4LC6 4LC8 4LP9 4LXQ 4LXT 4LXU 4LXX 4M0K 4M3S 4MAM 4MBY 4MDG 4MQL 4MUZ 4MZB 4N4B 4N6P 4N9P 4NBU 4O0A 


\section{Summary of the hydration site prediction results on two external data sets}

Table S2. Hydration site prediction success rates on Carlsson's data set by three methods

\begin{tabular}{|c|c|c|c|c|c|c|c|c|c|}
\hline \multirow{2}{*}{$\begin{array}{c}\text { Predictio } \\
\text { n } \\
\text { Criteria }^{*}\end{array}$} & \multicolumn{3}{|c|}{$\begin{array}{l}\text { Prediction success rate } \\
(\%) \text { of HydraMap on }\end{array}$} & \multicolumn{3}{|c|}{$\begin{array}{c}\text { Prediction success rate } \\
(\%) \text { of 3D-RISM on }\end{array}$} & \multicolumn{3}{|c|}{$\begin{array}{c}\text { Prediction success rate } \\
\text { (\%) of WATsite on }\end{array}$} \\
\hline & $\begin{array}{c}\text { Apo } \\
\text { protein } \\
\mathrm{S}\end{array}$ & $\begin{array}{l}\text { Holo } \\
\text { protein } \\
\mathrm{s}\end{array}$ & $\begin{array}{c}\text { All } \\
\text { protein } \\
\mathrm{S}\end{array}$ & $\begin{array}{c}\text { Apo } \\
\text { protein } \\
\mathrm{S}\end{array}$ & $\begin{array}{l}\text { Holo } \\
\text { protein } \\
\mathrm{s}\end{array}$ & $\begin{array}{c}\text { All } \\
\text { protein } \\
\mathrm{s}\end{array}$ & $\begin{array}{c}\text { Apo } \\
\text { protein } \\
\mathrm{S}\end{array}$ & $\begin{array}{l}\text { Holo } \\
\text { protein } \\
\mathrm{S}\end{array}$ & $\begin{array}{c}\text { All } \\
\text { protein } \\
\mathrm{S}\end{array}$ \\
\hline$<1.0 \AA$ & 207 & 2 & 00 & 38.6 & 46.9 & 43.0 & 46. & 45.1 & 45.8 \\
\hline$<1.5 \AA$ & 69.3 & 7 & 70.6 & 60.4 & 65.5 & 63.1 & 77.2 & 75.2 & 76.2 \\
\hline$<2.0 \AA$ & 92.1 & 94.7 & 93.5 & 79.2 & 86.7 & 83.2 & 88.1 & 85.0 & 86.4 \\
\hline
\end{tabular}

* Deviation between the predicted hydration site and observed water molecule in the binding pocket

Table S3. Total number of hydration sites predicted on Carlsson's data set by three methods

\begin{tabular}{|c|c|c|c|c|c|}
\hline \multirow{2}{*}{ Protein } & \multirow{2}{*}{ PDB code $^{\mathbf{a}}$} & \multirow{2}{*}{$\begin{array}{c}\text { Crystal } \\
\text { waters }\end{array}$} & \multicolumn{3}{|c|}{ Total No. of waters predicted by } \\
\cline { 4 - 6 } & & $5(13)$ & $23(20)$ & $15(13)$ & $16(18)$ \\
\hline Acetylcholinesterase & 1E66 (1EA5) & HydraMap & 3D-RISM & WATsite \\
\hline Coagulation factor VII & 1W7X (1KLJ) & $19(1)$ & $36(35)$ & $40(40)$ & $40(33)$ \\
\hline $\begin{array}{c}\text { Fatty acid binding } \\
\text { protein adipocyte }\end{array}$ & 2NNQ (3Q6L) & $7(16)$ & $46(44)$ & $27(23)$ & $22(24)$ \\
\hline $\begin{array}{c}\text { Glutamate ionotropic } \\
\text { receptor, AMPA subunit }\end{array}$ & 3KGC (4O3B) & $13(6)$ & $29(24)$ & $27(11)$ & $32(11)$ \\
\hline $\begin{array}{c}\text { Heat shock protein 90- } \\
\text { alpha }\end{array}$ & 1UYG (1UYL) & $11(14)$ & $35(22)$ & $20(14)$ & $23(18)$ \\
\hline $\begin{array}{c}\text { Tyrosine-protein kinase } \\
\text { JAK2 }\end{array}$ & 3LPB & 9 & 37 & 37 & 32 \\
\hline $\begin{array}{c}\text { Poly[ADP-ribose] } \\
\text { polymerase-1 }\end{array}$ & 3L3M & 4 & 26 & 21 & 25 \\
\hline $\begin{array}{c}\text { Serine/threonine-protein } \\
\text { kinase PLK1 }\end{array}$ & 2OWB & 10 & 36 & 32 & 31 \\
\hline $\begin{array}{c}\text { Protein-tyrosine } \\
\text { phosphatase 1B }\end{array}$ & 2AZR (2CM2) & $2(12)$ & $21(24)$ & $16(22)$ & $18(24)$ \\
\hline GAR transformylase & 1NJS & 12 & 38 & 38 & 34 \\
\hline $\begin{array}{c}\text { Muscle glycogen } \\
\text { phosphorylase }\end{array}$ & $1 \mathrm{C} 8 \mathrm{~K}$ & 6 & 31 & 25 & 23 \\
\hline $\begin{array}{c}\text { Thrombin } \\
\text { Trypsin I }\end{array}$ & 2AYWE (2UUF) & $5(20)$ & $37(33)$ & $30(32)$ & $32(26)$ \\
\hline
\end{tabular}

a PDB codes of reference crystal structures of the holo and apo (in parentheses) forms. ${ }^{b}$ Number of waters in the binding site of the crystal structure, i.e. within $4 \AA$ of both the protein and ligand. ${ }^{\mathrm{c}}$ Number of waters in 
the binding site of the crystal structure predicted by three methods.

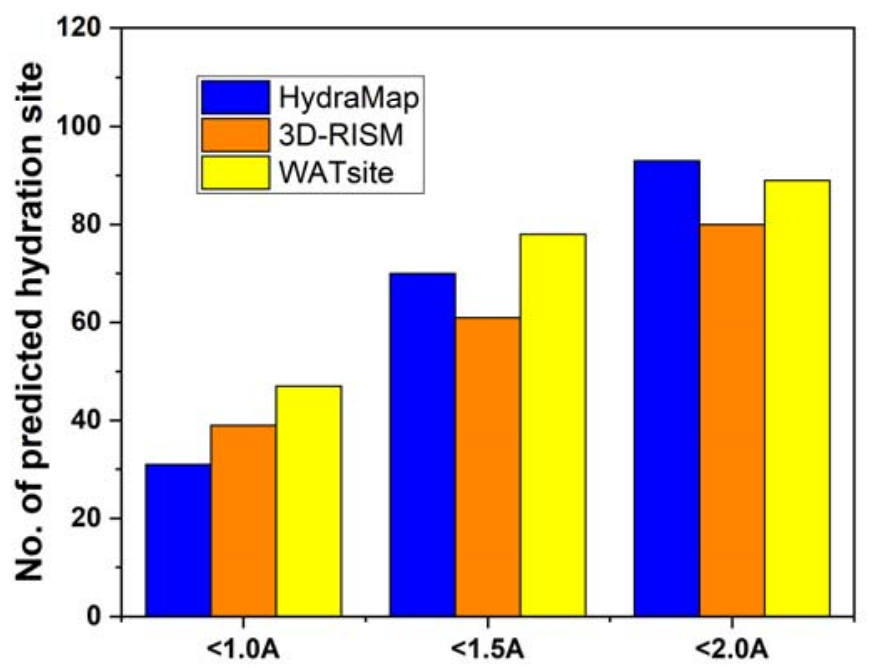

\section{Deviation between the predicted hydration site and} the water observed in crystal structure

Figure S3. The histogram of the deviation distribution of the predicted hydration sites compared to the crystal water molecule centroids of the apo structures in Carlsson's data set. The results of three methods, HydraMap, 3D-RISM and WATsite were provided.

Table S4. Hydration site prediction success rates on all 57 clusters of PDBbind core set by HydraMap and 3D-RISM

\begin{tabular}{|c|c|c|c|c|c|c|}
\hline \multirow{2}{*}{$\begin{array}{l}\text { Protein } \\
\text { Clusters }\end{array}$} & \multicolumn{3}{|l}{$\begin{array}{l}\text { Prediction success rate (\%) of HydraMap } \\
\text { at three criteria }\end{array}$} & \multicolumn{3}{l|}{$\begin{array}{l}\text { Prediction success rate (\%) of 3D-RISM } \\
\text { at three criteria }\end{array}$} \\
\cline { 2 - 7 } & $<\mathbf{1 . 0 \AA}$ & $<\mathbf{1 . 5 \AA}$ & $\mathbf{2 . 0 \AA}$ & $<\mathbf{1 . 0 \AA}$ & $<\mathbf{1 . 5 \AA}$ & $<\mathbf{2 . 0 \AA}$ \\
\hline 1 & 28.6 & 75.0 & 96.4 & 39.3 & 64.3 & 71.4 \\
\hline 2 & 44.0 & 88.0 & 100.0 & 52.0 & 64.0 & 80.0 \\
\hline 3 & 42.9 & 85.7 & 100.0 & 14.3 & 14.3 & 71.4 \\
\hline 4 & 26.4 & 64.2 & 83.0 & 32.1 & 54.7 & 71.7 \\
\hline 5 & 25.7 & 42.9 & 82.9 & 51.4 & 82.9 & 88.6 \\
\hline 6 & 31.6 & 57.9 & 89.5 & 31.6 & 52.6 & 68.4 \\
\hline 7 & 50.0 & 75.0 & 75.0 & 50.0 & 50.0 & 75.0 \\
\hline 8 & 38.9 & 72.2 & 88.9 & 38.9 & 50.0 & 88.9 \\
\hline 9 & 26.3 & 57.9 & 86.8 & 42.1 & 76.3 & 89.5 \\
\hline 10 & 70.0 & 100.0 & 100.0 & 60.0 & 90.0 & 100.0 \\
\hline 11 & 37.5 & 75.0 & 90.6 & 40.6 & 62.5 & 71.9 \\
\hline 12 & 45.5 & 59.1 & 90.9 & 36.4 & 72.7 & 90.9 \\
\hline 13 & 25.0 & 50.0 & 91.7 & 41.7 & 66.7 & 66.7 \\
\hline 14 & 35.0 & 90.0 & 95.0 & 45.0 & 75.0 & 90.0 \\
\hline 15 & 33.3 & 81.0 & 90.5 & 57.1 & 81.0 & 85.7 \\
\hline 16 & 35.0 & 65.0 & 100.0 & 45.0 & 60.0 & 75.0 \\
\hline
\end{tabular}




\begin{tabular}{|c|c|c|c|c|c|c|}
\hline 17 & 15.0 & 75.0 & 90.0 & 30.0 & 60.0 & 85.0 \\
\hline 18 & 26.7 & 73.3 & 93.3 & 26.7 & 60.0 & 73.3 \\
\hline 19 & 17.8 & 48.9 & 88.9 & 51.1 & 77.8 & 84.4 \\
\hline 20 & 5.3 & 57.9 & 89.5 & 21.1 & 57.9 & 78.9 \\
\hline 21 & 25.0 & 65.0 & 95.0 & 60.0 & 70.0 & 80.0 \\
\hline 22 & 22.7 & 68.2 & 86.4 & 36.4 & 68.2 & 90.9 \\
\hline 23 & 33.3 & 72.7 & 93.9 & 48.5 & 72.7 & 90.9 \\
\hline 24 & 33.3 & 79.2 & 100.0 & 33.3 & 62.5 & 87.5 \\
\hline 25 & 27.3 & 40.9 & 77.3 & 50.0 & 72.7 & 90.9 \\
\hline 26 & 42.9 & 81.0 & 95.2 & 23.8 & 42.9 & 85.7 \\
\hline 27 & 11.8 & 52.9 & 76.5 & 41.2 & 76.5 & 88.2 \\
\hline 28 & 20.5 & 65.9 & 93.2 & 43.2 & 68.2 & 84.1 \\
\hline 29 & 45.5 & 72.7 & 90.9 & 54.5 & 72.7 & 90.9 \\
\hline 30 & 24.1 & 65.5 & 93.1 & 37.9 & 79.3 & 100.0 \\
\hline 31 & 41.7 & 83.3 & 91.7 & 25.0 & 50.0 & 91.7 \\
\hline 32 & 33.3 & 66.7 & 88.9 & 72.2 & 72.2 & 83.3 \\
\hline 33 & 15.8 & 73.7 & 94.7 & 31.6 & 57.9 & 73.7 \\
\hline 34 & 42.4 & 76.3 & 89.8 & 47.5 & 71.2 & 83.1 \\
\hline 35 & 55.6 & 88.9 & 100.0 & 100.0 & 100.0 & 100.0 \\
\hline 36 & 31.6 & 84.2 & 94.7 & 57.9 & 73.7 & 73.7 \\
\hline 37 & 8.0 & 40.0 & 88.0 & 44.0 & 52.0 & 84.0 \\
\hline 38 & 34.4 & 59.4 & 87.5 & 43.8 & 53.1 & 71.9 \\
\hline 39 & 71.4 & 71.4 & 71.4 & 42.9 & 85.7 & 85.7 \\
\hline 40 & 25.0 & 72.2 & 94.4 & 44.4 & 75.0 & 88.9 \\
\hline 41 & 77.8 & 88.9 & 100.0 & 44.4 & 55.6 & 66.7 \\
\hline 42 & 33.3 & 73.3 & 90.0 & 53.3 & 66.7 & 86.7 \\
\hline 43 & 26.3 & 68.4 & 89.5 & 33.3 & 68.4 & 96.5 \\
\hline 44 & 38.9 & 72.2 & 94.4 & 83.3 & 94.4 & 94.4 \\
\hline 45 & 34.0 & 72.0 & 92.0 & 56.0 & 82.0 & 92.0 \\
\hline 46 & 13.8 & 58.6 & 82.8 & 37.9 & 51.7 & 58.6 \\
\hline 47 & 14.8 & 59.3 & 92.6 & 55.6 & 74.1 & 81.5 \\
\hline 48 & 57.1 & 85.7 & 85.7 & 28.6 & 57.1 & 85.7 \\
\hline 49 & 27.6 & 63.8 & 77.6 & 41.4 & 77.6 & 91.4 \\
\hline 50 & 26.7 & 73.3 & 93.3 & 20.0 & 73.3 & 80.0 \\
\hline 51 & 75.0 & 100.0 & 100.0 & 62.5 & 87.5 & 87.5 \\
\hline 52 & 13.3 & 66.7 & 100.0 & 53.3 & 86.7 & 100.0 \\
\hline 53 & 20.0 & 60.0 & 100.0 & 40.0 & 60.0 & 80.0 \\
\hline 54 & 28.6 & 77.1 & 94.3 & 40.0 & 71.4 & 91.4 \\
\hline 55 & 21.3 & 70.2 & 89.4 & 53.2 & 74.5 & 89.4 \\
\hline 56 & 28.6 & 81.0 & 97.6 & 47.6 & 76.2 & 90.5 \\
\hline 57 & 25.9 & 74.1 & 96.3 & 44.4 & 63.0 & 92.6 \\
\hline Total & 29.5 & 68.4 & 90.6 & 44.2 & 68.8 & 84.7 \\
\hline
\end{tabular}


Note: the clusters labeled in green, yellow and pink refer to the cases on which the hydration site prediction success rate of HydraMap is better, comparable to or worse than that of 3D-RISM, when the deviation between the predicted hydration site and the observed water molecule in the binding pocket is less than 1.50 $\AA$.

Table S5. Basic information of the PDBbind core set (v.2016)

\begin{tabular}{|c|c|c|c|c|}
\hline PDB Code & Resolution & $\mathbf{p K _ { d }} / \mathbf{p} \mathbf{K}_{\mathrm{i}}$ & $\begin{array}{c}\text { No. of water } \\
\text { molecules within } \\
4 \AA \AA \text { of the protein } \\
\text { and ligand atoms } \\
\text { in the crystal } \\
\text { structure }\end{array}$ & Target Cluster \\
\hline 1PS3 & 1.80 & 2.28 & 7 & \multirow{5}{*}{ Golgi alpha-mannosidase II } \\
\hline $3 \mathrm{D} 4 \mathrm{Z}$ & 1.39 & 4.89 & 4 & \\
\hline 3DX1 & 1.21 & 3.58 & 3 & \\
\hline $3 \mathrm{DX} 2$ & 1.40 & 6.82 & 4 & \\
\hline 3EJR & 1.27 & 8.57 & 10 & \\
\hline $3 \mathrm{EBP}$ & 2.00 & 5.91 & 4 & \multirow{5}{*}{ Glycogen phosphorylase } \\
\hline $3 \mathrm{G} 2 \mathrm{~N}$ & 2.10 & 4.09 & 5 & \\
\hline 3L7B & 2.00 & 2.40 & 5 & \\
\hline 3SYR & 2.40 & 5.10 & 4 & \\
\hline 4EKY & 2.45 & 3.52 & 7 & \\
\hline $2 \mathrm{VVN}$ & 1.85 & 7.30 & 3 & \multirow{5}{*}{ O-GLCNACASE BT_4395 } \\
\hline $2 \mathrm{~W} 4 \mathrm{X}$ & 2.42 & 4.85 & 1 & \\
\hline $2 \mathrm{~W} 66$ & 2.27 & 4.05 & 1 & \\
\hline $2 \mathrm{WCA}$ & 2.30 & 5.60 & 1 & \\
\hline $2 \mathrm{XJ} 7$ & 2.00 & 6.66 & 1 & \\
\hline 3ARP & 1.55 & 7.15 & 18 & \multirow{5}{*}{ Chitinase A } \\
\hline 3ARQ & 1.50 & 6.40 & 10 & \\
\hline 3ARU & 1.90 & 3.22 & 7 & \\
\hline $3 \mathrm{ARV}$ & 1.50 & 5.64 & 10 & \\
\hline 3ARY & 1.35 & 6.00 & 8 & \\
\hline $3 \mathrm{CJ} 4$ & 2.07 & 6.51 & 4 & \multirow{5}{*}{ RNA-directed RNA polymerase } \\
\hline 3GNW & 2.39 & 9.10 & 14 & \\
\hline $4 \mathrm{EO} 8$ & 1.80 & 8.15 & 5 & \\
\hline $4 \mathrm{IH} 5$ & 1.90 & 4.11 & 7 & \\
\hline $4 \mathrm{IH} 7$ & 2.30 & 5.24 & 5 & \\
\hline $1 \mathrm{E} 66$ & 2.10 & 9.89 & 5 & \multirow{5}{*}{ Acetylcholinesterase } \\
\hline $1 \mathrm{GPK}$ & 2.10 & 5.37 & 5 & \\
\hline $1 \mathrm{GPN}$ & 2.35 & 6.48 & 3 & \\
\hline $1 \mathrm{H} 22$ & 2.15 & 9.10 & 10 & \\
\hline $1 \mathrm{H} 23$ & 2.15 & 8.35 & 15 & \\
\hline
\end{tabular}




\begin{tabular}{|c|c|c|c|c|}
\hline $3 \mathrm{~F} 3 \mathrm{~A}$ & 2.00 & 4.19 & 3 & \multirow{5}{*}{ Transporter } \\
\hline $3 \mathrm{~F} 3 \mathrm{C}$ & 2.10 & 6.02 & 0 & \\
\hline $3 \mathrm{~F} 3 \mathrm{D}$ & 2.30 & 7.16 & 0 & \\
\hline $3 \mathrm{~F} 3 \mathrm{E}$ & 1.80 & 7.70 & 0 & \\
\hline 4MME & 2.50 & 6.50 & 1 & \\
\hline $2 \mathrm{CBV}$ & 1.95 & 5.48 & 3 & \multirow{5}{*}{ Beta-glucosidase A } \\
\hline 2CET & 1.97 & 8.02 & 3 & \\
\hline $2 \mathrm{~J} 78$ & 1.65 & 6.42 & 4 & \\
\hline $2 \mathrm{~J} 7 \mathrm{H}$ & 1.95 & 7.19 & 3 & \\
\hline $2 \mathrm{WBG}$ & 1.85 & 4.45 & 5 & \\
\hline $2 \mathrm{VKM}$ & 2.05 & 8.74 & 13 & \multirow{5}{*}{ Beta-secretase 1} \\
\hline 3RSX & 2.48 & 4.41 & 1 & \\
\hline $3 \mathrm{UDH}$ & 1.70 & 2.85 & 3 & \\
\hline 4DJV & 1.73 & 6.72 & 8 & \\
\hline 4GID & 2.00 & 10.77 & 13 & \\
\hline $2 \mathrm{WVT}$ & 1.80 & 6.12 & 2 & \multirow{5}{*}{ Alpha-L-fucosidase } \\
\hline 2XII & 1.80 & 7.20 & 3 & \\
\hline $4 \mathrm{~J} 28$ & 1.73 & 5.70 & 3 & \\
\hline 4JFS & 2.00 & 5.27 & 2 & \\
\hline $4 \mathrm{PCS}$ & 1.77 & 7.85 & 0 & \\
\hline $1 \mathrm{R} 5 \mathrm{Y}$ & 1.20 & 6.46 & 4 & \multirow{5}{*}{ tRNA guanine transglycosylase } \\
\hline $1 \mathrm{~S} 38$ & 1.81 & 5.15 & 5 & \\
\hline $3 \mathrm{GC} 5$ & 1.40 & 7.26 & 8 & \\
\hline $3 \mathrm{GE7}$ & 1.50 & 8.70 & 5 & \\
\hline 3RR4 & 1.68 & 4.55 & 10 & \\
\hline 2ZB1 & 2.50 & 6.32 & 2 & \multirow{5}{*}{$\begin{array}{c}\text { Mitogen-activated protein } \\
\text { kinase } 14\end{array}$} \\
\hline 3E92 & 2.00 & 8.00 & 1 & \\
\hline $3 \mathrm{E} 93$ & 2.00 & 8.85 & 10 & \\
\hline 4DLI & 1.91 & 5.62 & 5 & \\
\hline $4 \mathrm{~F} 9 \mathrm{~W}$ & 2.00 & 6.94 & 4 & \\
\hline 3QQS & 1.97 & 5.82 & 3 & \multirow{5}{*}{$\begin{array}{c}\text { Anthranilate } \\
\text { phosphoribosyltransferase }\end{array}$} \\
\hline $3 \mathrm{R} 88$ & 1.73 & 4.82 & 4 & \\
\hline 3TWP & 1.83 & 3.92 & 2 & \\
\hline 4GKM & 1.67 & 5.17 & 2 & \\
\hline 4OWM & 1.99 & 2.96 & 1 & \\
\hline 2R9W & 1.80 & 5.10 & 6 & \multirow{5}{*}{ Beta-lactamase } \\
\hline 3GR2 & 1.80 & 2.52 & 4 & \\
\hline 3GV9 & 1.80 & 2.12 & 1 & \\
\hline 4JXS & 1.90 & 4.74 & 5 & \\
\hline $4 \mathrm{KZ6}$ & 1.68 & 3.10 & 4 & \\
\hline $3 \mathrm{UI} 7$ & 2.28 & 9.00 & 3 & \multirow{3}{*}{$\begin{array}{l}\text { cAMP and cAMP-inhibited } \\
\text { cGMP 3',5'-cyclic } \\
\text { phosphodiesterase 10A }\end{array}$} \\
\hline 3UUO & 2.11 & 7.96 & 2 & \\
\hline 4LLX & 1.75 & 2.89 & 4 & \\
\hline
\end{tabular}




\begin{tabular}{|c|c|c|c|c|}
\hline $5 \mathrm{C} 28$ & 1.56 & 5.66 & 5 & \\
\hline $5 \mathrm{C} 2 \mathrm{H}$ & 2.09 & 11.09 & 7 & \\
\hline $1 \mathrm{BZC}$ & 2.35 & 4.92 & 1 & \multirow{5}{*}{$\begin{array}{c}\text { Tyrosine-protein phosphatase } \\
\text { non-receptor type } 1\end{array}$} \\
\hline $2 \mathrm{HB} 1$ & 2.00 & 3.80 & 2 & \\
\hline 2QBP & 2.50 & 8.40 & 3 & \\
\hline 2QBQ & 2.10 & 7.44 & 10 & \\
\hline $2 \mathrm{QBR}$ & 2.30 & 6.33 & 4 & \\
\hline 1Q8T & 2.00 & 4.76 & 4 & \multirow{5}{*}{$\begin{array}{l}\text { cAMP-dependent protein } \\
\text { kinase, alpha-catalytic subunit }\end{array}$} \\
\hline $1 \mathrm{Q} 8 \mathrm{U}$ & 1.90 & 5.96 & 3 & \\
\hline $1 Y D R$ & 2.20 & 5.52 & 3 & \\
\hline $1 Y \mathrm{YDT}$ & 2.30 & 7.32 & 5 & \\
\hline 3AG9 & 2.00 & 8.05 & 5 & \\
\hline $2 \mathrm{C} 3 \mathrm{I}$ & 1.90 & 7.60 & 3 & \multirow{5}{*}{$\begin{array}{c}\text { Proto-oncogene } \\
\text { serine/threonine-protein kinase } \\
\text { Pim-1 }\end{array}$} \\
\hline $3 \mathrm{BGZ}$ & 2.40 & 6.26 & 3 & \\
\hline 3JYA & 2.10 & 6.89 & 0 & \\
\hline $4 \mathrm{~K} 18$ & 2.05 & 8.96 & 4 & \\
\hline 5DWR & 2.00 & 11.22 & 5 & \\
\hline $2 \mathrm{~V} 00$ & 1.55 & 3.66 & 7 & \multirow{5}{*}{ Endothiapepsin } \\
\hline 3PRS & 1.38 & 7.82 & 10 & \\
\hline 3PWW & 1.22 & 7.32 & 10 & \\
\hline 3URI & 2.10 & 9.00 & 10 & \\
\hline $3 \mathrm{WZ} 8$ & 1.45 & 5.82 & 8 & \\
\hline 1QF1 & 2.00 & 7.32 & 2 & \multirow{5}{*}{ Thermolysin } \\
\hline $1 \mathrm{Z9G}$ & 1.70 & 5.64 & 0 & \\
\hline $3 \mathrm{FCQ}$ & 1.75 & 2.77 & 4 & \\
\hline $4 \mathrm{TMN}$ & 1.70 & 10.17 & 4 & \\
\hline $5 \mathrm{TMN}$ & 1.60 & 8.04 & 9 & \\
\hline $3 \mathrm{COY}$ & 2.03 & 6.02 & 4 & \multirow{5}{*}{ Pantothenate synthetase } \\
\hline $3 \mathrm{COZ}$ & 2.00 & 5.57 & 5 & \\
\hline $3 \mathrm{IVG}$ & 1.95 & 4.30 & 5 & \\
\hline $4 \mathrm{DDH}$ & 2.07 & 3.32 & 3 & \\
\hline 4DDK & 1.75 & 2.29 & 3 & \\
\hline $1 \mathrm{PXN}$ & 2.50 & 7.15 & 0 & \multirow{5}{*}{ Cell division protein kinase 2} \\
\hline 2FVD & 1.85 & 8.52 & 12 & \\
\hline $2 \mathrm{XNB}$ & 1.85 & 6.83 & 6 & \\
\hline $3 \mathrm{PXF}$ & 1.80 & 4.43 & 0 & \\
\hline 4EOR & 2.20 & 6.30 & 4 & \\
\hline $4 \mathrm{E} 5 \mathrm{~W}$ & 1.86 & 7.66 & 11 & \multirow{5}{*}{ Tyrosine-protein kinase JAK1 } \\
\hline $4 I V B$ & 1.90 & 8.72 & 6 & \\
\hline $4 \mathrm{IVC}$ & 2.35 & 10.00 & 3 & \\
\hline 4IVD & 1.93 & 9.52 & 7 & \\
\hline $4 \mathrm{~K} 77$ & 2.40 & 6.63 & 6 & \\
\hline 4E6Q & 1.95 & 8.36 & 1 & Tyrosine-protein kinase JAK2 \\
\hline
\end{tabular}




\begin{tabular}{|c|c|c|c|c|}
\hline 4F09 & 2.40 & 6.70 & 5 & \\
\hline 4GFM & 2.30 & 7.22 & 6 & \\
\hline 4HGE & 2.30 & 7.92 & 5 & \\
\hline 4JIA & 1.85 & 9.22 & 7 & \\
\hline $1 \mathrm{NVQ}$ & 2.00 & 8.25 & 5 & \multirow{5}{*}{$\begin{array}{l}\text { Serine/Threonine-protein kinase } \\
\text { Chk1 }\end{array}$} \\
\hline 2BR1 & 2.00 & 5.14 & 9 & \\
\hline 2BRB & 2.10 & 4.86 & 3 & \\
\hline 3JVR & 1.76 & 5.72 & 2 & \\
\hline $3 J V S$ & 1.90 & 6.54 & 3 & \\
\hline 2ZCQ & 2.38 & 8.82 & 3 & \multirow{5}{*}{ Dehydrosqualene synthase } \\
\hline $2 \mathrm{ZCR}$ & 1.92 & 6.87 & 8 & \\
\hline $2 \mathrm{ZY} 1$ & 1.78 & 7.40 & 8 & \\
\hline $3 \mathrm{ACW}$ & 1.63 & 4.76 & 1 & \\
\hline 4EA2 & 2.05 & 6.44 & 1 & \\
\hline $2 \mathrm{~V} 7 \mathrm{~A}$ & 2.50 & 8.30 & 7 & \multirow{5}{*}{ Tyrosine-protein kinase ABL1 } \\
\hline $3 \mathrm{~K} 5 \mathrm{~V}$ & 1.74 & 6.30 & 4 & \\
\hline $3 \mathrm{MSS}$ & 1.95 & 4.66 & 1 & \\
\hline 3PYY & 1.85 & 6.86 & 2 & \\
\hline 4TWP & 2.40 & 10.00 & 3 & \\
\hline $1 \mathrm{BCU}$ & 2.00 & 3.28 & 3 & \multirow{5}{*}{ thrombin light chain } \\
\hline $1 \mathrm{OYT}$ & 1.67 & 7.24 & 11 & \\
\hline $2 \mathrm{ZDA}$ & 1.73 & 8.40 & 11 & \\
\hline 3BV9 & 1.80 & 5.36 & 12 & \\
\hline $3 \mathrm{UTU}$ & 1.55 & 10.92 & 7 & \\
\hline $2 \mathrm{P} 4 \mathrm{Y}$ & 2.25 & 9.00 & 1 & \multirow{5}{*}{$\begin{array}{l}\text { Peroxisome proliferator- } \\
\text { activated receptor gamma }\end{array}$} \\
\hline $2 \mathrm{YFE}$ & 2.00 & 6.63 & 1 & \\
\hline $3 \mathrm{~B} 1 \mathrm{M}$ & 1.60 & 8.48 & 6 & \\
\hline 3FUR & 2.30 & 8.00 & 2 & \\
\hline 3U9Q & 1.52 & 4.38 & 1 & \\
\hline $2 \mathrm{WTV}$ & 2.40 & 8.74 & 6 & \multirow{5}{*}{ Aurora kinase A } \\
\hline $3 \mathrm{E} 5 \mathrm{~A}$ & 2.30 & 8.23 & 4 & \\
\hline $3 \mathrm{MYG}$ & 2.40 & 10.70 & 6 & \\
\hline $3 \mathrm{UO} 4$ & 2.45 & 6.52 & 2 & \\
\hline $3 \mathrm{UP} 2$ & 2.30 & 7.40 & 11 & \\
\hline 3QGY & 2.10 & 7.80 & 4 & \multirow{5}{*}{$\begin{array}{c}\text { Tyrosine-protein kinase } \\
\text { ITK/TSK }\end{array}$} \\
\hline $4 \mathrm{M} 0 \mathrm{Y}$ & 1.70 & 6.46 & 2 & \\
\hline $4 \mathrm{M} 0 \mathrm{Z}$ & 2.00 & 5.19 & 2 & \\
\hline 4QD6 & 2.45 & 8.64 & 1 & \\
\hline 4RFM & 2.10 & 10.05 & 3 & \\
\hline $1 \mathrm{C} 5 \mathrm{Z}$ & 1.85 & 4.01 & 3 & \multirow{4}{*}{$\begin{array}{l}\text { Urokinase-type plasminogen } \\
\text { activator }\end{array}$} \\
\hline $105 \mathrm{~B}$ & 1.85 & 5.77 & 4 & \\
\hline $10 W H$ & 1.61 & 7.40 & 6 & \\
\hline 1SQA & 2.00 & 9.21 & 2 & \\
\hline
\end{tabular}




\begin{tabular}{|c|c|c|c|c|}
\hline $3 \mathrm{KGP}$ & 2.35 & 2.57 & 3 & \\
\hline 2WEG & 1.10 & 6.50 & 1 & \multirow{5}{*}{ Carbonic anhydrase 2} \\
\hline 3DD0 & 1.48 & 9.00 & 5 & \\
\hline $3 \mathrm{KWA}$ & 2.00 & 4.08 & 3 & \\
\hline $3 \mathrm{RYJ}$ & 1.39 & 7.80 & 8 & \\
\hline 4JSZ & 1.90 & 2.30 & 2 & \\
\hline $1 \mathrm{YC} 1$ & 1.70 & 6.17 & 14 & \multirow{5}{*}{$\begin{array}{l}\text { Heat shock protein HSP 90- } \\
\text { alpha }\end{array}$} \\
\hline $2 \mathrm{XDL}$ & 1.98 & 3.10 & 10 & \\
\hline $2 \mathrm{YKI}$ & 1.67 & 9.46 & 12 & \\
\hline $3 \mathrm{~B} 27$ & 1.50 & 5.16 & 14 & \\
\hline 3RLR & 1.70 & 7.52 & 9 & \\
\hline $1 \mathrm{Z95}$ & 1.80 & 7.12 & 2 & \multirow{5}{*}{ Androgen receptor } \\
\hline 3B5R & 1.80 & 8.77 & 2 & \\
\hline $3 \mathrm{~B} 65$ & 1.80 & 9.27 & 2 & \\
\hline $3 \mathrm{~B} 68$ & 1.90 & 8.40 & 2 & \\
\hline $3 \mathrm{G} 0 \mathrm{~W}$ & 1.95 & 9.52 & 1 & \\
\hline $1 \mathrm{P} 1 \mathrm{~N}$ & 1.60 & 6.80 & 5 & \multirow{5}{*}{ Glutamate receptor 2} \\
\hline $1 \mathrm{P} 1 \mathrm{Q}$ & 2.00 & 4.89 & 3 & \\
\hline 1SYI & 2.10 & 5.44 & 2 & \\
\hline $2 \mathrm{AL} 5$ & 1.65 & 8.40 & 4 & \\
\hline $4 \mathrm{U} 4 \mathrm{~S}$ & 1.90 & 2.92 & 5 & \\
\hline $3 \mathrm{G} 2 \mathrm{Z}$ & 1.50 & 2.36 & 6 & \multirow{5}{*}{ Beta-lactamase (CTX-M-9) } \\
\hline $3 \mathrm{G} 31$ & 1.70 & 2.89 & 2 & \\
\hline 4DE1 & 1.26 & 5.96 & 5 & \\
\hline 4DE2 & 1.40 & 4.12 & 7 & \\
\hline 4DE3 & 1.44 & 5.52 & 5 & \\
\hline $1 \mathrm{VSO}$ & 1.85 & 4.72 & 8 & \multirow{5}{*}{$\begin{array}{l}\text { Glutamate receptor, ionotropic } \\
\text { kainate } 1\end{array}$} \\
\hline $3 \mathrm{FV} 1$ & 1.50 & 9.30 & 3 & \\
\hline $3 \mathrm{FV} 2$ & 1.50 & 8.11 & 4 & \\
\hline $3 \mathrm{GBB}$ & 2.10 & 6.90 & 6 & \\
\hline 4DLD & 2.00 & 5.82 & 11 & \\
\hline $1 \mathrm{QKT}$ & 2.20 & 9.04 & 2 & \multirow{5}{*}{ Estrogen receptor } \\
\hline $2 \mathrm{P} 15$ & 1.94 & 10.30 & 2 & \\
\hline $2 \mathrm{POG}$ & 1.84 & 9.54 & 1 & \\
\hline 2QE4 & 2.40 & 7.96 & 1 & \\
\hline 4MGD & 1.90 & 4.69 & 1 & \\
\hline $1 \mathrm{LPG}$ & 2.00 & 7.09 & 8 & \multirow{5}{*}{$\begin{array}{c}\text { Coagulation Factor X Heavy } \\
\text { Chain }\end{array}$} \\
\hline 1MQ6 & 2.10 & 11.15 & 5 & \\
\hline $1 \mathrm{Z6E}$ & 1.80 & 9.72 & 4 & \\
\hline $2 \mathrm{XBV}$ & 1.66 & 8.43 & 9 & \\
\hline $2 \mathrm{Y} 5 \mathrm{H}$ & 1.33 & 5.79 & 10 & \\
\hline $1 \mathrm{NC} 1$ & 2.00 & 6.12 & 2 & \multirow{2}{*}{ MTA/SAH nucleosidase } \\
\hline 1NC3 & 2.20 & 5.00 & 3 & \\
\hline
\end{tabular}




\begin{tabular}{|c|c|c|c|c|}
\hline 1Y6R & 2.20 & 10.11 & 1 & \\
\hline $4 \mathrm{~F} 2 \mathrm{~W}$ & 2.00 & 11.30 & 1 & \\
\hline $4 \mathrm{~F} 3 \mathrm{C}$ & 1.93 & 11.82 & 2 & \\
\hline $1 \mathrm{~K} 1 \mathrm{I}$ & 2.20 & 6.58 & 8 & \multirow{5}{*}{ Beta-Trypsin } \\
\hline $1 \mathrm{O} 3 \mathrm{~F}$ & 1.55 & 7.96 & 7 & \\
\hline $1 \mathrm{UTO}$ & 1.15 & 2.27 & 6 & \\
\hline $3 \mathrm{GY} 4$ & 1.55 & 5.10 & 4 & \\
\hline $4 \mathrm{ABG}$ & 1.52 & 3.57 & 5 & \\
\hline $2 \mathrm{FXS}$ & 2.00 & 6.06 & 16 & \multirow{5}{*}{$\begin{array}{l}\text { ATP-dependent molecular } \\
\text { chaperone HSP82 }\end{array}$} \\
\hline 2IWX & 1.50 & 6.68 & 12 & \\
\hline 2VW5 & 1.90 & 8.52 & 10 & \\
\hline 2WER & 1.60 & 7.05 & 6 & \\
\hline $2 \mathrm{YGE}$ & 1.96 & 5.06 & 13 & \\
\hline 3KR8 & 2.10 & 8.10 & 5 & \multirow{5}{*}{ Tankyrase-2 } \\
\hline $4 \mathrm{~J} 21$ & 1.93 & 7.41 & 3 & \\
\hline $4 \mathrm{~J} 3 \mathrm{~L}$ & 2.09 & 7.80 & 5 & \\
\hline $4 K Z Q$ & 2.25 & 6.10 & 2 & \\
\hline $4 \mathrm{KZU}$ & 2.10 & 6.50 & 3 & \\
\hline 4CR9 & 1.70 & 4.10 & 5 & \multirow{5}{*}{ Coagulation Factor XI } \\
\hline 4CRA & 1.80 & 7.22 & 11 & \\
\hline 4CRC & 1.60 & 8.72 & 9 & \\
\hline $4 \mathrm{TY} 7$ & 2.09 & 9.52 & 11 & \\
\hline $4 \mathrm{X} 6 \mathrm{P}$ & 1.93 & 8.30 & 14 & \\
\hline $2 \mathrm{WN} 9$ & 1.75 & 8.52 & 3 & \multirow{5}{*}{ Soluble acetylcholine receptor } \\
\hline $2 \mathrm{WNC}$ & 2.20 & 6.32 & 8 & \\
\hline $2 \times 00$ & 2.40 & 11.33 & 10 & \\
\hline $2 \mathrm{XYS}$ & 1.91 & 7.42 & 7 & \\
\hline 2YMD & 1.96 & 3.16 & 1 & \\
\hline 3NW9 & 1.65 & 9.00 & 6 & \multirow{5}{*}{ Catechol O-methyltransferase } \\
\hline $3 \mathrm{OE} 4$ & 1.49 & 7.47 & 3 & \\
\hline $3 \mathrm{OE} 5$ & 1.52 & 6.88 & 6 & \\
\hline $3 \mathrm{OZS}$ & 1.44 & 5.33 & 6 & \\
\hline $3 \mathrm{OZT}$ & 1.48 & 4.13 & 6 & \\
\hline $3 \mathrm{U} 8 \mathrm{~K}$ & 2.47 & 8.66 & 1 & \multirow{5}{*}{ Acetylcholine-binding protein } \\
\hline $3 \mathrm{U} 8 \mathrm{~N}$ & 2.35 & 10.17 & 2 & \\
\hline $3 \mathrm{WTJ}$ & 2.24 & 6.53 & 1 & \\
\hline $3 Z \mathrm{ZG}$ & 2.48 & 7.10 & 2 & \\
\hline 4QAC & 2.10 & 9.40 & 1 & \\
\hline $4 \mathrm{AGN}$ & 1.60 & 3.97 & 13 & \multirow{5}{*}{ Cellular Tumor Antigen P53 } \\
\hline 4AGP & 1.50 & 4.69 & 9 & \\
\hline 4AGQ & 1.42 & 5.01 & 13 & \\
\hline 5A7B & 1.40 & 3.57 & 13 & \\
\hline $5 \mathrm{ABA}$ & 1.62 & 2.98 & 10 & \\
\hline
\end{tabular}




\begin{tabular}{|c|c|c|c|c|}
\hline $3 \mathrm{AO} 4$ & 1.95 & 2.07 & 2 & \multirow{5}{*}{ Integrase } \\
\hline $3 \mathrm{ZSO}$ & 1.75 & 5.12 & 5 & \\
\hline $3 Z S X$ & 1.95 & 3.28 & 2 & \\
\hline $3 \mathrm{ZT} 2$ & 1.70 & 2.84 & 2 & \\
\hline $4 \mathrm{CIG}$ & 1.70 & 3.67 & 4 & \\
\hline $2 \mathrm{XB} 8$ & 2.40 & 7.59 & 2 & \multirow{5}{*}{ 3-dehydroquinate dehydratase } \\
\hline $3 N 76$ & 1.90 & 6.85 & 2 & \\
\hline 3N7A & 2.00 & 3.70 & 0 & \\
\hline $3 \mathrm{~N} 86$ & 1.90 & 5.64 & 2 & \\
\hline 4CIW & 2.20 & 4.82 & 2 & \\
\hline 4BKT & 2.35 & 3.62 & 1 & \multirow{5}{*}{$\begin{array}{l}\text { Transcription elongation factor } \\
\text { B polypeptide } 2\end{array}$} \\
\hline 4W9C & 2.20 & 4.65 & 2 & \\
\hline 4W9H & 2.10 & 6.73 & 5 & \\
\hline 4W9I & 2.40 & 5.96 & 4 & \\
\hline 4W9L & 2.20 & 5.02 & 3 & \\
\hline 3NQ9 & 1.90 & 4.03 & 3 & \multirow{5}{*}{ Beta-lactoglobulin } \\
\hline 3UEU & 2.10 & 5.24 & 0 & \\
\hline 3UEV & 1.90 & 5.89 & 1 & \\
\hline 3UEW & 2.00 & 6.31 & 0 & \\
\hline 3UEX & 2.10 & 6.92 & 1 & \\
\hline 3EHY & 1.90 & 5.85 & 5 & \multirow{5}{*}{ Macrophage metalloelastase } \\
\hline 3LKA & 1.80 & 2.82 & 4 & \\
\hline $3 \mathrm{NX} 7$ & 1.80 & 8.10 & 8 & \\
\hline 3TSK & 2.00 & 7.17 & 7 & \\
\hline 4GR0 & 1.50 & 9.55 & 11 & \\
\hline $1 \mathrm{O} 0 \mathrm{H}$ & 1.20 & 5.92 & 14 & \multirow{5}{*}{$\begin{array}{l}\text { Bromodomain-containing } \\
\text { protein } 4\end{array}$} \\
\hline 1U1B & 2.00 & 7.80 & 3 & \\
\hline $1 \mathrm{~W} 4 \mathrm{O}$ & 1.60 & 5.22 & 6 & \\
\hline 3D6Q & 1.60 & 3.76 & 9 & \\
\hline $3 \mathrm{DXG}$ & 1.39 & 2.40 & 10 & \\
\hline $1 \mathrm{~A} 30$ & 2.00 & 4.30 & 5 & \multirow{5}{*}{ Pancreatic Ribonuclease A } \\
\hline $1 \mathrm{EBY}$ & 2.29 & 9.70 & 7 & \\
\hline 2QNQ & 2.30 & 6.11 & 3 & \\
\hline 3O9I & 1.45 & 11.82 & 8 & \\
\hline $1 \mathrm{G} 2 \mathrm{~K}$ & 1.95 & 7.96 & 4 & \\
\hline $3 \mathrm{P} 5 \mathrm{O}$ & 1.60 & 7.30 & 13 & \multirow{5}{*}{ HIV-1 Protease } \\
\hline $3 \mathrm{U} 5 \mathrm{~J}$ & 1.60 & 5.61 & 7 & \\
\hline 4LZS & 2.20 & 4.80 & 9 & \\
\hline 4OGJ & 1.65 & 6.79 & 12 & \\
\hline 4WIV & 1.56 & 6.26 & 6 & \\
\hline
\end{tabular}


PART 2. Basic information of the data sets used for calibrating and validating the derivative scoring functions

Table S6. List of the protein-ligand complexes formed by AchE (39 in total)

\begin{tabular}{|c|c|c|c|c|c|c|c|c|}
\hline $\begin{array}{l}\text { PDB } \\
\text { Code }\end{array}$ & $\begin{array}{l}\text { Resolution } \\
(\AA)\end{array}$ & $\begin{array}{l}\mathrm{pK}_{\mathrm{d}} / \mathrm{pK}_{\mathrm{i}} \\
/ \mathrm{pIC}_{50}\end{array}$ & $\begin{array}{l}\text { PDB } \\
\text { Code }\end{array}$ & $\begin{array}{l}\text { Resolution } \\
(\AA)\end{array}$ & $\begin{array}{l}\mathrm{pK}_{\mathrm{d}} / \mathrm{pK}_{\mathrm{i}} \\
/ \mathrm{pIC}_{50}\end{array}$ & $\begin{array}{l}\text { PDB } \\
\text { Code }\end{array}$ & $\begin{array}{l}\text { Resolution } \\
(\AA)\end{array}$ & $\begin{array}{l}\mathrm{pK}_{\mathrm{d}} / \mathrm{pK}_{\mathrm{i}} \\
/ \mathrm{pIC}_{50}\end{array}$ \\
\hline 1DX6 & 2.30 & 6.19 & $2 \mathrm{CMF}$ & 2.50 & 7.55 & $3 Z \mathrm{ZV} 7$ & 2.26 & 5.92 \\
\hline 1EVE & 2.50 & 8.24 & $2 \mathrm{HA0}$ & 2.20 & 4.43 & $4 \mathrm{~A} 23$ & 2.40 & 5.89 \\
\hline 1HBJ & 2.50 & 8.52 & 2HA2 & 2.05 & 4.66 & 4ARA & 2.50 & 6.05 \\
\hline $1 \mathrm{~J} 07$ & 2.35 & 7.68 & 2HA3 & 2.25 & 3.03 & 4ARB & 2.25 & 5.89 \\
\hline 1N5R & 2.25 & 5.66 & 2HA5 & 2.15 & 3.21 & 4B7Z & 2.30 & 4.31 \\
\hline 1Q84 & 2.45 & 11.05 & $2 \mathrm{HA6}$ & 2.25 & 4.32 & $4 \mathrm{~B} 80$ & 2.50 & 4.40 \\
\hline 1QTI & 2.50 & 5.68 & $2 \mathrm{~W} 9 \mathrm{I}$ & 2.43 & 7.48 & $4 \mathrm{~B} 82$ & 2.10 & 4.39 \\
\hline $1 \mathrm{VOT}$ & 2.50 & 6.60 & $2 \mathrm{WHP}$ & 2.20 & 4.00 & 4B83 & 2.40 & 5.15 \\
\hline $1 \mathrm{~W} 4 \mathrm{~L}$ & 2.16 & 8.40 & $2 \mathrm{WIG}$ & 2.15 & 3.43 & 4B85 & 2.10 & 4.06 \\
\hline 1W6R & 2.05 & 6.15 & $2 \mathrm{WIJ}$ & 2.10 & 3.20 & 4BDS & 2.10 & 7.60 \\
\hline $1 \mathrm{~W} 76$ & 2.30 & 6.15 & $2 \mathrm{WIK}$ & 2.10 & 3.18 & 4BTL & 2.50 & 5.60 \\
\hline $1 \mathrm{ZGB}$ & 2.30 & 8.06 & $3 \mathrm{I} 6 \mathrm{M}$ & 2.26 & 6.46 & $4 \mathrm{M} 0 \mathrm{E}$ & 2.00 & 6.22 \\
\hline $2 \mathrm{CKM}$ & 2.15 & 8.82 & $3 \mathrm{I} 6 \mathrm{Z}$ & 2.19 & 7.30 & $4 \mathrm{M} 0 \mathrm{~F}$ & 2.30 & 8.77 \\
\hline
\end{tabular}

Table S7. List of BACE-1 protein-ligand complexes (218 in total)

\begin{tabular}{|c|c|c|c|c|c|c|c|c|}
\hline $\begin{array}{l}\text { PDB } \\
\text { Code } \\
\end{array}$ & $\begin{array}{l}\text { Resolution } \\
(\AA)\end{array}$ & $\begin{array}{l}\mathrm{pK}_{\mathrm{d}} / \mathrm{pK}_{\mathrm{i}} \\
/ \mathrm{pIC}_{50}\end{array}$ & $\begin{array}{l}\text { PDB } \\
\text { Code }\end{array}$ & $\begin{array}{l}\text { Resolution } \\
(\AA)\end{array}$ & $\begin{array}{l}\mathrm{pK}_{\mathrm{d}} / \mathrm{pK}_{\mathrm{i}} \\
/ \mathrm{pIC}_{50}\end{array}$ & $\begin{array}{l}\text { PDB } \\
\text { Code } \\
\end{array}$ & $\begin{array}{l}\text { Resolution } \\
(\AA)\end{array}$ & $\begin{array}{l}\mathrm{pK}_{\mathrm{d}} / \mathrm{pK}_{\mathrm{i}} \\
/ \mathrm{pIC}_{50}\end{array}$ \\
\hline $1 \mathrm{FKN}$ & 1.90 & 8.80 & 3HW1 & 2.48 & 3.06 & 4B1D & 1.95 & 7.49 \\
\hline $1 \mathrm{M} 4 \mathrm{H}$ & 2.10 & 9.52 & $3 \mathrm{I} 25$ & 2.10 & 8.51 & $4 \mathrm{~B} 70$ & 1.60 & 5.20 \\
\hline $1 \mathrm{TQF}$ & 1.80 & 5.85 & 3 IGB & 2.24 & 4.42 & 4B72 & 1.60 & 6.20 \\
\hline 1YM2 & 2.05 & 8.00 & 3IN3 & 2.00 & 7.22 & 4B77 & 1.80 & 5.10 \\
\hline 1YM4 & 2.25 & 7.41 & 3IN4 & 2.30 & 7.52 & 4B78 & 1.50 & 5.00 \\
\hline 2B8L & 1.70 & 7.82 & 3IND & 2.25 & 5.82 & 4BEK & 2.39 & 4.39 \\
\hline $2 \mathrm{~B} 8 \mathrm{~V}$ & 1.80 & 7.01 & 3INE & 2.00 & 6.77 & 4BFD & 2.30 & 7.02 \\
\hline $2 \mathrm{~F} 3 \mathrm{E}$ & 2.11 & 6.81 & 3INF & 1.85 & 7.40 & 4D83 & 2.40 & 7.26 \\
\hline $2 \mathrm{~F} 3 \mathrm{~F}$ & 2.30 & 6.72 & $3 \mathrm{INH}$ & 1.80 & 7.70 & 4D88 & 1.70 & 7.31 \\
\hline $2 \mathrm{FDP}$ & 2.50 & 7.59 & $3 \mathrm{IVH}$ & 1.80 & 7.33 & 4D89 & 1.65 & 8.70 \\
\hline $2 \mathrm{G} 94$ & 1.86 & 9.52 & 3 IVI & 2.20 & 7.92 & $4 \mathrm{D} 8 \mathrm{C}$ & 2.07 & 8.70 \\
\hline $2 \mathrm{HIZ}$ & 2.50 & 6.95 & 3IXJ & 2.20 & 9.49 & 4DH6 & 2.50 & 7.77 \\
\hline 2HM1 & 2.20 & 8.70 & 3IXK & 2.50 & 8.18 & 4DI2 & 2.00 & 8.26 \\
\hline 2IQG & 1.70 & 8.30 & $3 \mathrm{~K} 5 \mathrm{C}$ & 2.12 & 7.77 & 4DJU & 1.80 & 5.44 \\
\hline 2IRZ & 1.80 & 7.92 & $3 \mathrm{~K} 5 \mathrm{~F}$ & 2.25 & 5.43 & 4DJW & 1.90 & 6.28 \\
\hline 2IS0 & 2.20 & 6.70 & $3 \mathrm{~K} 5 \mathrm{G}$ & 2.00 & 8.60 & 4DJX & 1.50 & 7.23 \\
\hline
\end{tabular}




\begin{tabular}{|c|c|c|c|c|c|c|c|c|}
\hline $2 \mathrm{OAH}$ & 1.80 & 7.96 & $3 \mathrm{KMX}$ & 1.70 & 4.82 & 4DJY & 1.86 & 8.27 \\
\hline $2 \mathrm{OHP}$ & 2.25 & 4.03 & $3 \mathrm{KMY}$ & 1.90 & 4.49 & 4DPF & 1.80 & 6.40 \\
\hline 2OHQ & 2.10 & 4.60 & $3 \mathrm{KN} 0$ & 1.90 & 3.60 & 4DPI & 1.90 & 7.11 \\
\hline 2OHR & 2.25 & 4.00 & $3 \mathrm{~L} 38$ & 2.10 & 7.00 & 4DUS & 2.50 & 8.30 \\
\hline 2OHS & 2.45 & 4.40 & 3L3A & 2.36 & 6.38 & 4DV9 & 2.08 & 6.72 \\
\hline $2 \mathrm{OHT}$ & 2.30 & 5.04 & 3L58 & 1.80 & 8.10 & 4DVF & 1.80 & 6.89 \\
\hline $2 \mathrm{OHU}$ & 2.35 & 5.38 & 3L59 & 2.00 & 3.70 & 4EWO & 1.80 & 7.36 \\
\hline $2 \mathrm{P} 4 \mathrm{~J}$ & 2.50 & 8.96 & 3L5B & 1.80 & 3.91 & $4 \mathrm{EXG}$ & 1.80 & 8.07 \\
\hline $2 \mathrm{P} 83$ & 2.50 & 7.96 & 3L5C & 1.80 & 5.15 & 4FGX & 1.59 & 7.14 \\
\hline $2 \mathrm{P} 8 \mathrm{H}$ & 1.80 & 7.15 & 3L5D & 1.75 & 4.11 & 4FM7 & 1.56 & 7.24 \\
\hline 2PH6 & 2.00 & 7.57 & $3 \mathrm{~L} 5 \mathrm{E}$ & 1.53 & 7.57 & 4FM8 & 1.90 & 5.98 \\
\hline $2 \mathrm{PH} 8$ & 1.70 & 5.74 & $3 \mathrm{~L} 5 \mathrm{~F}$ & 1.70 & 6.22 & 4FRI & 2.30 & 5.55 \\
\hline 2Q11 & 2.40 & 6.05 & $3 \mathrm{LHG}$ & 2.10 & 7.70 & 4FRJ & 1.95 & 6.55 \\
\hline $2 \mathrm{Q} 15$ & 2.40 & 7.96 & $3 \mathrm{LNK}$ & 1.80 & 5.94 & 4FRK & 2.10 & 8.10 \\
\hline 2QK5 & 2.20 & 8.10 & 3LPI & 2.05 & 8.52 & 4FRS & 1.70 & 8.77 \\
\hline 2QMD & 1.65 & 8.30 & 3LPJ & 1.79 & 6.92 & 4FS4 & 1.74 & 6.57 \\
\hline 2QMF & 1.75 & 8.52 & $3 \mathrm{LPK}$ & 1.93 & 9.10 & 4FSL & 1.70 & 7.70 \\
\hline 2QMG & 1.89 & 9.15 & $3 \mathrm{MSJ}$ & 1.80 & 3.11 & $4 \mathrm{H} 1 \mathrm{E}$ & 1.90 & 8.52 \\
\hline 2QP8 & 1.50 & 8.10 & $3 \mathrm{MSK}$ & 2.00 & 4.59 & $4 \mathrm{H} 3 \mathrm{~F}$ & 1.70 & 9.00 \\
\hline 2QU3 & 2.00 & 6.23 & 3MSL & 2.40 & 5.15 & $4 \mathrm{H} 3 \mathrm{G}$ & 1.85 & 8.22 \\
\hline 2QZK & 1.80 & 7.57 & $3 \mathrm{NSH}$ & 2.20 & 6.33 & $4 \mathrm{H} 3 \mathrm{I}$ & 1.96 & 8.52 \\
\hline 2QZL & 1.80 & 8.10 & $3 \mathrm{OHF}$ & 2.10 & 7.03 & $4 \mathrm{H} 3 \mathrm{~J}$ & 1.60 & 7.05 \\
\hline 2VA6 & 2.50 & 6.17 & $3 \mathrm{OHH}$ & 2.01 & 7.74 & 4HA5 & 1.83 & 7.24 \\
\hline 2VA7 & 2.20 & 6.70 & $300 Z$ & 1.80 & 7.85 & 4HZT & 1.80 & 6.12 \\
\hline $2 \mathrm{VIE}$ & 1.90 & 7.48 & 3 PI5 & 2.40 & 6.02 & $4 \mathrm{I} 0 \mathrm{D}$ & 1.91 & 6.21 \\
\hline $2 \mathrm{VIY}$ & 1.82 & 5.74 & $3 \mathrm{QBH}$ & 2.24 & 6.82 & $4 \mathrm{I} 0 \mathrm{~F}$ & 1.80 & 6.35 \\
\hline $2 \mathrm{VIZ}$ & 1.60 & 6.22 & 3QI1 & 2.30 & 7.66 & $4 \mathrm{I} 10$ & 2.07 & 6.77 \\
\hline 2VJ6 & 1.80 & 7.89 & $3 \mathrm{RSV}$ & 2.50 & 9.15 & $4 \mathrm{I} 11$ & 1.89 & 4.57 \\
\hline $2 \mathrm{VJ} 7$ & 1.60 & 7.40 & 3RU1 & 2.30 & 4.85 & $4 \mathrm{I} 12$ & 1.78 & 6.39 \\
\hline $2 \mathrm{VJ} 9$ & 1.60 & 6.74 & $3 \mathrm{~S} 7 \mathrm{~L}$ & 2.16 & 7.10 & $4 \mathrm{I} 1 \mathrm{C}$ & 2.00 & 8.10 \\
\hline $2 \mathrm{VNM}$ & 1.79 & 8.52 & $3 \mathrm{~S} 7 \mathrm{M}$ & 2.20 & 8.00 & 4IVT & 1.60 & 6.00 \\
\hline $2 \mathrm{VNN}$ & 1.87 & 8.70 & 3ТPP & 1.60 & 7.82 & $4 \mathrm{JOP}$ & 1.97 & 7.29 \\
\hline 2WEZ & 1.70 & 7.64 & $3 \mathrm{U} 6 \mathrm{~A}$ & 2.20 & 6.49 & 4J0T & 2.05 & 6.74 \\
\hline $2 \mathrm{WF} 0$ & 1.60 & 6.68 & 3UDJ & 1.80 & 3.62 & $4 \mathrm{~J} 0 \mathrm{~V}$ & 1.94 & 7.55 \\
\hline 2WF1 & 1.60 & 8.70 & $3 \mathrm{UDM}$ & 1.94 & 4.48 & $4 \mathrm{JOY}$ & 1.77 & 6.36 \\
\hline $2 \mathrm{WF} 2$ & 1.80 & 7.59 & $3 \mathrm{UDN}$ & 2.19 & 5.15 & $4 \mathrm{~J} 0 \mathrm{Z}$ & 2.13 & 7.11 \\
\hline 2WF3 & 2.08 & 7.72 & 3UDP & 1.95 & 5.22 & $4 \mathrm{~J} 17$ & 1.81 & 7.27 \\
\hline $2 \mathrm{WF} 4$ & 1.80 & 7.89 & 3UDR & 1.95 & 4.96 & $4 \mathrm{~J} 1 \mathrm{C}$ & 2.01 & 7.31 \\
\hline $2 \mathrm{XFI}$ & 1.73 & 7.82 & $3 \mathrm{UDY}$ & 2.00 & 5.70 & $4 \mathrm{~J} 1 \mathrm{E}$ & 1.78 & 7.72 \\
\hline $2 \mathrm{XFJ}$ & 1.80 & 7.89 & 3UFL & 1.90 & 6.82 & $4 \mathrm{~J} 1 \mathrm{~F}$ & 2.25 & 7.92 \\
\hline $2 \mathrm{XFK}$ & 1.80 & 8.52 & $3 \mathrm{UQP}$ & 1.77 & 7.31 & $4 \mathrm{~J} 1 \mathrm{H}$ & 2.20 & 6.11 \\
\hline $2 \mathrm{ZDZ}$ & 2.00 & 6.15 & $3 \mathrm{VEU}$ & 1.52 & 7.26 & $4 \mathrm{~J} 1 \mathrm{I}$ & 2.05 & 7.40 \\
\hline 2ZE1 & 2.20 & 6.22 & 3VF3 & 1.48 & 5.86 & $4 \mathrm{~J} 1 \mathrm{~K}$ & 2.18 & 7.89 \\
\hline
\end{tabular}




\begin{tabular}{|l|l|l|l|l|l|l|l|l|}
\hline 3BRA & 2.30 & 2.70 & 3VG1 & 1.77 & 7.26 & 4JOO & 1.80 & 4.43 \\
\hline 3BUF & 2.30 & 3.10 & 3VV6 & 2.05 & 3.80 & 4JP9 & 1.80 & 7.62 \\
\hline 3BUG & 2.50 & 3.18 & 3VV7 & 2.10 & 5.34 & 4JPC & 1.80 & 7.03 \\
\hline 3BUH & 2.30 & 3.66 & 3VV8 & 2.50 & 4.77 & 4JPE & 1.80 & 7.32 \\
\hline 3CIB & 1.72 & 7.85 & 3WB4 & 2.25 & 4.44 & 4K8S & 2.39 & 7.44 \\
\hline 3CIC & 1.75 & 8.52 & 3WB5 & 2.50 & 4.57 & 4K9H & 2.29 & 7.12 \\
\hline 3CID & 1.80 & 8.30 & 3ZMG & 1.74 & 7.64 & 4KE0 & 2.30 & 7.77 \\
\hline 3CKP & 2.30 & 6.35 & 3ZOV & 2.10 & 7.22 & 4KE1 & 1.91 & 8.60 \\
\hline 3DUY & 1.97 & 5.85 & 4ACU & 1.75 & 7.39 & 4L7G & 1.38 & 4.00 \\
\hline 3DV1 & 2.10 & 6.23 & 4ACX & 2.00 & 7.11 & 4LC7 & 1.70 & 4.93 \\
\hline 3DV5 & 2.10 & 7.66 & 4AZY & 1.79 & 6.70 & 4LXA & 1.95 & 8.70 \\
\hline 3EXO & 2.10 & 4.62 & 4B00 & 1.83 & 8.61 & 4LXK & 2.05 & 8.40 \\
\hline 3FKT & 1.90 & 5.55 & 4B05 & 1.80 & 7.58 & 4LXM & 2.30 & 7.06 \\
\hline 3HVG & 2.26 & 2.70 & 4B1C & 1.95 & 7.10 & & & \\
\hline
\end{tabular}

Table S8. List of FXa protein-ligand complexes (79 in total)

\begin{tabular}{|c|c|c|c|c|c|c|c|c|}
\hline $\begin{array}{l}\text { PDB } \\
\text { Code }\end{array}$ & $\begin{array}{l}\text { Resolution } \\
(\AA)\end{array}$ & $\begin{array}{l}\mathrm{pK}_{\mathrm{d}} / \mathrm{pK}_{\mathrm{i}} \\
/ \mathrm{pIC}_{50}\end{array}$ & $\begin{array}{l}\text { PDB } \\
\text { Code }\end{array}$ & $\begin{array}{l}\text { Resolution } \\
(\AA)\end{array}$ & $\begin{array}{l}\mathrm{pK}_{\mathrm{d}} / \mathrm{pK}_{\mathrm{i}} \\
/ \mathrm{pIC}_{50}\end{array}$ & $\begin{array}{l}\text { PDB } \\
\text { Code }\end{array}$ & $\begin{array}{l}\text { Resolution } \\
(\AA)\end{array}$ & $\begin{array}{l}\mathrm{pK}_{\mathrm{d}} / \mathrm{pK}_{\mathrm{i}} \\
/ \mathrm{pIC}_{50}\end{array}$ \\
\hline $1 \mathrm{EZQ}$ & 2.20 & 9.05 & $2 \mathrm{JKH}$ & 1.25 & 8.05 & $2 \mathrm{XC} 0$ & 2.05 & 6.72 \\
\hline 1F0R & 2.10 & 7.66 & $2 \mathrm{P} 16$ & 2.30 & 10.10 & $2 \mathrm{XC4}$ & 1.67 & 8.05 \\
\hline $1 \mathrm{~F} 0 \mathrm{~S}$ & 2.10 & 7.74 & $2 \mathrm{P} 93$ & 1.90 & 8.82 & $2 \mathrm{Y} 5 \mathrm{~F}$ & 1.29 & 8.70 \\
\hline 1FJS & 1.92 & 9.96 & 2P94 & 1.80 & 9.17 & $2 \mathrm{Y} 5 \mathrm{G}$ & 1.29 & 6.84 \\
\hline $1 \mathrm{G} 2 \mathrm{~L}$ & 1.90 & 7.24 & 2P95 & 2.20 & 9.37 & $2 \mathrm{Y} 7 \mathrm{X}$ & 1.90 & 8.89 \\
\hline $1 \mathrm{KSN}$ & 2.10 & 9.40 & 2PHB & 2.30 & 9.49 & $2 Y 7 Z$ & 1.84 & 8.70 \\
\hline $1 \mathrm{LPK}$ & 2.20 & 7.55 & 2Q1J & 1.90 & 9.10 & $2 Y 80$ & 1.90 & 8.05 \\
\hline $1 \mathrm{LPZ}$ & 2.40 & 7.60 & 2RA0 & 2.30 & 7.80 & $2 Y 81$ & 1.70 & 8.70 \\
\hline $1 \mathrm{MQ5}$ & 2.10 & 9.00 & 2UWL & 1.90 & 8.40 & $2 Y 82$ & 2.20 & 8.40 \\
\hline $1 \mathrm{NFU}$ & 2.05 & 7.74 & 2UWO & 1.75 & 8.70 & 3 CEN & 1.60 & 8.26 \\
\hline 1NFW & 2.10 & 8.96 & 2UWP & 1.76 & 6.81 & $3 \mathrm{CS} 7$ & 2.20 & 10.68 \\
\hline 1NFX & 2.15 & 8.52 & $2 \mathrm{VH} 0$ & 1.70 & 8.51 & 3ENS & 2.30 & 8.05 \\
\hline 1NFY & 2.10 & 8.89 & 2VH6 & 1.95 & 9.70 & 3FFG & 1.54 & 9.08 \\
\hline $1 \mathrm{~V} 3 \mathrm{X}$ & 2.20 & 7.62 & $2 \mathrm{VVC}$ & 1.95 & 8.52 & 3HPT & 2.19 & 8.19 \\
\hline 1WU1 & 2.30 & 7.74 & $2 \mathrm{VVU}$ & 2.30 & 8.10 & 3K9X & 1.90 & 8.40 \\
\hline 1XKA & 2.30 & 6.88 & $2 \mathrm{VVV}$ & 1.73 & 8.22 & 3KL6 & 1.45 & 8.46 \\
\hline $2 \mathrm{BOH}$ & 2.20 & 8.52 & $2 \mathrm{VWL}$ & 1.80 & 7.02 & $3 \mathrm{KQB}$ & 2.25 & 9.30 \\
\hline $2 \mathrm{BOK}$ & 1.64 & 6.55 & 2VWM & 1.96 & 7.03 & $3 \mathrm{KQC}$ & 2.20 & 8.66 \\
\hline $2 \mathrm{CJI}$ & 2.10 & 8.22 & $2 \mathrm{VWN}$ & 1.61 & 8.00 & $3 \mathrm{KQE}$ & 2.35 & 8.09 \\
\hline 2EI6 & 2.30 & 7.39 & $2 \mathrm{VWO}$ & 1.60 & 7.51 & 3LIW & 2.22 & 7.60 \\
\hline $2 \mathrm{FZZ}$ & 2.20 & 10.52 & $2 W 26$ & 2.08 & 9.40 & 3M36 & 2.15 & 9.82 \\
\hline $2 \mathrm{G} 00$ & 2.10 & 9.74 & 2W3I & 1.90 & 10.00 & 3M37 & 1.90 & 9.04 \\
\hline $2 \mathrm{~J} 2 \mathrm{U}$ & 1.90 & 7.33 & $2 \mathrm{~W} 3 \mathrm{~K}$ & 2.05 & 10.10 & 3Q3K & 2.00 & 8.13 \\
\hline $2 \mathrm{~J} 34$ & 2.01 & 7.82 & $2 \mathrm{WYG}$ & 1.88 & 8.70 & 3SW2 & 2.42 & 8.33 \\
\hline 2J4I & 1.80 & 9.00 & $2 \mathrm{WYJ}$ & 2.38 & 9.00 & 4A7I & 2.40 & 8.70 \\
\hline
\end{tabular}




\begin{tabular}{|l|l|l|l|l|l|l|l|l|}
\hline 2J94 & 2.10 & 6.27 & 2XBW & 1.72 & 8.00 & & & \\
\hline 2J95 & 2.01 & 8.40 & 2XBX & 1.85 & 7.82 & & & \\
\hline
\end{tabular}

Table S9. List of HIV-1 protease protein-ligand complexes (172 in total)

\begin{tabular}{|c|c|c|c|c|c|c|c|c|}
\hline $\begin{array}{l}\text { PDB } \\
\text { Code }\end{array}$ & $\begin{array}{l}\text { Resolution } \\
(\AA)\end{array}$ & $\begin{array}{l}\mathrm{pK}_{\mathrm{d}} / \mathrm{pK}_{\mathrm{i}} \\
/ \mathrm{pIC}_{50}\end{array}$ & $\begin{array}{l}\text { PDB } \\
\text { Code }\end{array}$ & $\begin{array}{l}\text { Resolution } \\
(\AA)\end{array}$ & $\begin{array}{l}\mathrm{pK}_{\mathrm{d}} / \mathrm{pK}_{\mathrm{i}} \\
/ \mathrm{pIC}_{50}\end{array}$ & $\begin{array}{l}\text { PDB } \\
\text { Code }\end{array}$ & $\begin{array}{l}\text { Resolution } \\
(\AA)\end{array}$ & $\begin{array}{l}\mathrm{pK}_{\mathrm{d}} / \mathrm{pK}_{\mathrm{i}} \\
/ \mathrm{pIC}_{50}\end{array}$ \\
\hline 1A94 & 2.00 & 7.85 & 1PRO & 1.80 & 11.30 & 2QI7 & 1.85 & 10.21 \\
\hline 1AID & 2.20 & 4.82 & 1QBR & 1.80 & 10.57 & $2 \mathrm{QNN}$ & 1.48 & 7.15 \\
\hline $1 \mathrm{AJV}$ & 2.00 & 7.72 & 1QBS & 1.80 & 9.47 & 2QNP & 1.41 & 6.41 \\
\hline $1 \mathrm{AJX}$ & 2.00 & 7.91 & 1QBT & 2.10 & 10.62 & $2 U X Z$ & 1.75 & 8.48 \\
\hline $1 \mathrm{~B} 6 \mathrm{~J}$ & 1.85 & 7.92 & $1 \mathrm{QBU}$ & 1.80 & 10.24 & $2 \mathrm{UY0}$ & 1.76 & 6.92 \\
\hline 1B6K & 1.85 & 8.74 & $1 \mathrm{SBG}$ & 2.30 & 7.74 & $2 \mathrm{WKZ}$ & 1.70 & 8.77 \\
\hline 1B6L & 1.75 & 8.30 & $1 \mathrm{VIJ}$ & 2.40 & 9.52 & 2XYE & 2.00 & 8.34 \\
\hline 1B6M & 1.85 & 8.40 & $1 \mathrm{~W} 5 \mathrm{~V}$ & 1.80 & 8.15 & $2 X Y F$ & 1.80 & 8.44 \\
\hline 1BV7 & 2.00 & 10.30 & $1 \mathrm{~W} 5 \mathrm{~W}$ & 1.80 & 8.80 & $2 \mathrm{Z4O}$ & 1.60 & 9.57 \\
\hline $1 \mathrm{C} 70$ & 2.50 & 10.30 & $1 \mathrm{~W} 5 \mathrm{X}$ & 1.90 & 8.40 & $3 \mathrm{~A} 2 \mathrm{O}$ & 0.88 & 9.08 \\
\hline $1 \mathrm{CPI}$ & 2.05 & 7.41 & $1 \mathrm{~W} 5 \mathrm{Y}$ & 1.90 & 8.48 & 3AID & 2.50 & 6.86 \\
\hline 1D4H & 1.81 & 10.00 & $1 \mathrm{Z1H}$ & 1.85 & 8.40 & 3BGB & 1.90 & 6.05 \\
\hline 1D4I & 1.81 & 8.85 & $1 \mathrm{Z1R}$ & 1.85 & 9.22 & $3 \mathrm{BGC}$ & 1.80 & 5.02 \\
\hline 1D4J & 1.81 & 8.36 & $1 \mathrm{ZP} 8$ & 2.02 & 8.77 & $3 \mathrm{BXS}$ & 1.60 & 5.10 \\
\hline $1 \mathrm{D} 4 \mathrm{~K}$ & 1.85 & 9.22 & $1 \mathrm{ZPA}$ & 2.02 & 8.40 & 3CKT & 1.65 & 6.32 \\
\hline 1D4L & 1.75 & 8.77 & $1 \mathrm{ZSF}$ & 1.98 & 9.92 & 3DJK & 1.00 & 10.59 \\
\hline $1 \mathrm{DIF}$ & 1.70 & 10.66 & 1ZSR & 2.06 & 9.82 & 3DK1 & 1.07 & 9.74 \\
\hline 1DMP & 2.00 & 9.55 & $2 \mathrm{AOD}$ & 1.40 & 5.66 & 3EKX & 1.97 & 9.41 \\
\hline 1EBW & 1.81 & 9.05 & $2 \mathrm{AVV}$ & 1.50 & 9.26 & 3EL1 & 1.70 & 9.64 \\
\hline $1 \mathrm{EBZ}$ & 2.01 & 9.40 & 2BBB & 1.70 & 8.62 & $3 \mathrm{GI} 4$ & 1.85 & 10.80 \\
\hline $1 \mathrm{EC} 0$ & 1.79 & 8.49 & 2BPV & 1.90 & 7.67 & $3 \mathrm{GI} 5$ & 1.80 & 11.22 \\
\hline $1 \mathrm{EC} 1$ & 2.10 & 8.92 & 2BPY & 1.90 & 7.40 & $3 \mathrm{GI} 6$ & 1.84 & 11.22 \\
\hline $1 \mathrm{EC} 2$ & 2.00 & 10.00 & 2BQV & 2.10 & 8.05 & $3 \mathrm{H} 5 \mathrm{~B}$ & 1.29 & 10.00 \\
\hline $1 \mathrm{EC} 3$ & 1.80 & 9.04 & 2CEJ & 2.50 & 8.62 & $3 \mathrm{I} 6 \mathrm{O}$ & 1.17 & 10.35 \\
\hline $1 \mathrm{G} 35$ & 1.80 & 8.14 & 2CEM & 1.80 & 7.92 & $3 \mathrm{I7E}$ & 1.70 & 10.85 \\
\hline $1 \mathrm{GNO}$ & 2.30 & 7.70 & 2CEN & 1.70 & 8.30 & $3 \mathrm{KDB}$ & 1.66 & 9.85 \\
\hline $1 \mathrm{HBV}$ & 2.30 & 6.37 & $2 \mathrm{~F} 3 \mathrm{~K}$ & 1.60 & 7.89 & $3 \mathrm{KDC}$ & 2.20 & 9.28 \\
\hline $1 \mathrm{HEF}$ & 2.20 & 9.00 & 2FDD & 1.58 & 8.32 & $3 \mathrm{KDD}$ & 1.80 & 8.46 \\
\hline 1HEG & 2.20 & 7.74 & $2 \mathrm{FGV}$ & 1.50 & 6.12 & $3 \mathrm{M} 9 \mathrm{~F}$ & 1.80 & 11.15 \\
\hline $1 \mathrm{HIH}$ & 2.20 & 8.05 & 2FLE & 1.90 & 7.85 & $3 \mathrm{MXD}$ & 1.95 & 8.83 \\
\hline $1 \mathrm{HOS}$ & 2.30 & 8.55 & 2HB3 & 1.35 & 11.35 & $3 \mathrm{MXE}$ & 1.85 & 10.01 \\
\hline $1 \mathrm{HPO}$ & 2.50 & 9.22 & $2 \mathrm{I} 0 \mathrm{~A}$ & 1.80 & 11.40 & 3NU3 & 1.02 & 9.82 \\
\hline 1HPS & 2.30 & 9.22 & 2I0D & 1.95 & 12.10 & $30 K 9$ & 1.27 & 11.23 \\
\hline 1HPX & 2.00 & 11.26 & $2 \mathrm{I} 4 \mathrm{D}$ & 1.50 & 11.68 & 3QAA & 1.40 & 11.54 \\
\hline $1 \mathrm{HTF}$ & 2.20 & 6.83 & $2 \mathrm{I} 4 \mathrm{~W}$ & 1.55 & 11.59 & 3ST5 & 1.45 & 11.30 \\
\hline 1HTG & 2.00 & 8.42 & 2IEN & 1.30 & 9.00 & 3ТH9 & 1.34 & 7.70 \\
\hline $1 \mathrm{HVH}$ & 1.80 & 7.96 & $2 \mathrm{NXL}$ & 2.00 & 6.27 & $3 \mathrm{TLH}$ & 2.00 & 8.82 \\
\hline
\end{tabular}




\begin{tabular}{|c|c|c|c|c|c|c|c|c|}
\hline 1HVI & 1.80 & 10.92 & $2 \mathrm{NXM}$ & 2.25 & 5.19 & 3ZPT & 1.54 & 8.51 \\
\hline $1 \mathrm{HVJ}$ & 2.00 & 11.40 & $2 \mathrm{O} 4 \mathrm{P}$ & 1.80 & 10.72 & $3 Z \mathrm{PU}$ & 1.80 & 8.38 \\
\hline $1 \mathrm{HVK}$ & 1.80 & 10.96 & 2P3B & 2.10 & 8.48 & $4 \mathrm{~A} 4 \mathrm{Q}$ & 1.80 & 9.10 \\
\hline $1 \mathrm{HVL}$ & 1.90 & 9.95 & $2 \mathrm{PK} 5$ & 1.90 & 10.70 & 4A6B & 1.80 & 8.38 \\
\hline 1HVR & 1.80 & 9.51 & 2PK6 & 1.45 & 10.89 & 4A6C & 1.50 & 7.92 \\
\hline 1HWR & 1.80 & 8.33 & 2PQZ & 1.55 & 5.67 & $4 \mathrm{DFG}$ & 1.23 & 11.74 \\
\hline $1 \mathrm{HXB}$ & 2.30 & 9.92 & $2 \mathrm{PSU}$ & 1.93 & 7.62 & 4DJO & 1.78 & 11.52 \\
\hline $1 \mathrm{HXW}$ & 1.80 & 10.82 & 2PSV & 1.75 & 7.24 & 4DJP & 1.40 & 10.55 \\
\hline 1IIQ & 1.83 & 7.48 & 2PWC & 1.78 & 6.57 & 4DJQ & 1.40 & 10.32 \\
\hline $1 \mathrm{IZH}$ & 1.90 & 7.70 & 2PWR & 1.50 & 6.59 & 4DJR & 1.55 & 11.52 \\
\hline $1 \mathrm{~K} 6 \mathrm{P}$ & 2.20 & 7.36 & $2 \mathrm{Q} 54$ & 1.85 & 9.01 & 4EP2 & 1.90 & 5.35 \\
\hline $1 \mathrm{~K} 6 \mathrm{~T}$ & 2.25 & 7.62 & $2 \mathrm{Q} 55$ & 1.90 & 8.69 & 4GYE & 2.27 & 6.85 \\
\hline $1 \mathrm{~K} 6 \mathrm{~V}$ & 2.00 & 6.92 & 2Q5K & 1.95 & 11.30 & 4GZF & 2.05 & 8.36 \\
\hline $1 \mathrm{KZK}$ & 1.09 & 10.39 & $2 \mathrm{QHY}$ & 1.85 & 7.48 & $4 \mathrm{I} 8 \mathrm{~W}$ & 1.96 & 10.90 \\
\hline $1 \mathrm{M} 0 \mathrm{~B}$ & 2.45 & 8.82 & $2 \mathrm{QHZ}$ & 1.85 & 7.28 & $4 \mathrm{I} 8 \mathrm{Z}$ & 1.75 & 11.05 \\
\hline $1 \mathrm{MRW}$ & 2.00 & 9.70 & 2QI0 & 2.10 & 7.38 & $4 \mathrm{~KB} 9$ & 1.29 & 11.00 \\
\hline $1 \mathrm{NH} 0$ & 1.03 & 9.74 & 2QI1 & 2.00 & 7.30 & 4PHV & 2.10 & 9.17 \\
\hline $1 \mathrm{NPA}$ & 2.00 & 8.70 & 2QI3 & 1.95 & 10.20 & 5HVP & 2.00 & 7.70 \\
\hline $1 \mathrm{NPV}$ & 2.00 & 8.24 & 2QI4 & 1.80 & 10.44 & 7UPJ & 2.00 & 8.49 \\
\hline 1NPW & 2.00 & 8.70 & 2QI5 & 1.85 & 10.85 & & & \\
\hline 1ODY & 2.00 & 8.10 & 2QI6 & 1.85 & 10.57 & & & \\
\hline
\end{tabular}

Table S10. List of MAPK14 protein-ligand complexes (116 in total)

\begin{tabular}{|c|c|c|c|c|c|c|c|c|}
\hline $\begin{array}{l}\text { PDB } \\
\text { Code }\end{array}$ & $\begin{array}{l}\text { Resolution } \\
(\AA)\end{array}$ & $\begin{array}{l}\mathrm{pK}_{\mathrm{d}} / \mathrm{pK}_{\mathrm{i}} \\
/ \mathrm{pIC}_{50}\end{array}$ & $\begin{array}{l}\text { PDB } \\
\text { Code }\end{array}$ & $\begin{array}{l}\text { Resolution } \\
(\AA)\end{array}$ & $\begin{array}{l}\mathrm{pK}_{\mathrm{d}} / \mathrm{pK}_{\mathrm{i}} \\
/ \mathrm{pIC}_{50}\end{array}$ & $\begin{array}{l}\text { PDB } \\
\text { Code }\end{array}$ & $\begin{array}{l}\text { Resolution } \\
(\AA)\end{array}$ & $\begin{array}{l}\mathrm{pK}_{\mathrm{d}} / \mathrm{pK}_{\mathrm{i}} \\
/ \mathrm{pIC}_{50}\end{array}$ \\
\hline $1 \mathrm{~A} 9 \mathrm{U}$ & 2.50 & 7.32 & 3D7Z & 2.10 & 7.92 & 3NWW & 2.09 & 8.15 \\
\hline 1BL6 & 2.50 & 6.80 & 3D83 & 1.90 & 8.36 & $308 \mathrm{P}$ & 2.10 & 6.46 \\
\hline 1BL7 & 2.50 & 7.72 & $3 \mathrm{FCl}$ & 2.00 & 8.00 & $3 \mathrm{OCG}$ & 2.21 & 8.52 \\
\hline $1 \mathrm{BMK}$ & 2.40 & 7.60 & $3 \mathrm{GC7}$ & 1.80 & 9.22 & $3 \mathrm{P} 5 \mathrm{~K}$ & 2.09 & 7.12 \\
\hline $1 \mathrm{KV} 1$ & 2.50 & 5.94 & $3 \mathrm{GCP}$ & 2.25 & 7.82 & 3P78 & 2.30 & 6.96 \\
\hline $1 \mathrm{M} 7 \mathrm{Q}$ & 2.40 & 8.59 & $3 \mathrm{GCQ}$ & 2.00 & 6.47 & 3P79 & 2.10 & 5.64 \\
\hline 1OUK & 2.50 & 9.89 & $3 \mathrm{GCS}$ & 2.10 & 7.25 & 3P7A & 2.31 & 6.28 \\
\hline 1OUY & 2.50 & 8.37 & $3 \mathrm{GCU}$ & 2.10 & 6.78 & 3P7B & 1.90 & 7.74 \\
\hline 1OVE & 2.10 & 9.13 & $3 \mathrm{GCV}$ & 2.30 & 7.13 & $3 \mathrm{P} 7 \mathrm{C}$ & 2.30 & 7.66 \\
\hline $1 \mathrm{~W} 82$ & 2.20 & 6.71 & $3 \mathrm{GFE}$ & 2.10 & 8.49 & 3RIN & 2.20 & 8.89 \\
\hline $1 \mathrm{~W} 83$ & 2.50 & 7.19 & 3HEC & 2.50 & 4.47 & $3 \mathrm{ROC}$ & 1.70 & 8.19 \\
\hline $1 \mathrm{~W} 84$ & 2.20 & 4.46 & $3 \mathrm{HEG}$ & 2.20 & 6.74 & $3 \mathrm{~S} 3 \mathrm{I}$ & 1.80 & 7.80 \\
\hline $1 \mathrm{WBN}$ & 2.40 & 6.46 & 3HL7 & 1.88 & 7.64 & $3 \mathrm{~S} 4 \mathrm{Q}$ & 2.27 & 8.40 \\
\hline $1 \mathrm{WBS}$ & 1.80 & 6.20 & 3HLL & 1.95 & 8.24 & $3 \mathrm{U} 8 \mathrm{~W}$ & 2.15 & 9.00 \\
\hline $1 \mathrm{WBT}$ & 2.00 & 6.47 & 3HP2 & 2.15 & 6.17 & $3 U V P$ & 2.40 & 7.46 \\
\hline $1 \mathrm{WBV}$ & 2.00 & 3.79 & 3HP5 & 2.30 & 7.15 & 3UVQ & 2.20 & 7.77 \\
\hline $1 \mathrm{WBW}$ & 2.41 & 4.36 & $3 \mathrm{HRB}$ & 2.20 & 7.68 & 3ZYA & 1.90 & 7.00 \\
\hline 1YQJ & 2.00 & 8.14 & 3HUB & 2.25 & 8.09 & 4AA0 & 1.80 & 7.46 \\
\hline
\end{tabular}




\begin{tabular}{|l|l|l|l|l|l|l|l|l|}
\hline 1YW2 & 2.01 & 6.34 & 3HUC & 1.80 & 5.99 & 4AA4 & 2.30 & 8.05 \\
\hline 1ZYJ & 2.00 & 5.82 & 3HV3 & 2.00 & 6.33 & 4AA5 & 2.38 & 7.80 \\
\hline 1ZZ2 & 2.00 & 5.22 & 3HV5 & 2.25 & 7.02 & 4AAC & 2.50 & 8.17 \\
\hline 1ZZL & 2.00 & 8.30 & 3HV6 & 1.95 & 5.64 & 4EH2 & 2.00 & 3.16 \\
\hline 2BAJ & 2.25 & 8.40 & 3HV7 & 2.40 & 7.92 & 4EH3 & 2.40 & 3.53 \\
\hline 2BAK & 2.20 & 7.43 & 3HVC & 2.10 & 6.22 & 4EH4 & 2.50 & 2.81 \\
\hline 2BAL & 2.10 & 6.31 & 3IPH & 2.10 & 7.60 & 4EH5 & 2.00 & 3.09 \\
\hline 2EWA & 2.10 & 7.94 & 3ITZ & 2.25 & 8.85 & 4EH6 & 2.10 & 2.46 \\
\hline 2GFS & 1.75 & 6.15 & 3IW5 & 2.50 & 6.08 & 4EH7 & 2.10 & 3.28 \\
\hline 2I0H & 2.00 & 8.05 & 3IW6 & 2.10 & 5.48 & 4EH8 & 2.20 & 2.20 \\
\hline 2QD9 & 1.70 & 7.89 & 3IW8 & 2.00 & 4.87 & 4EH9 & 2.10 & 2.26 \\
\hline 2RG5 & 2.40 & 8.51 & 3K3I & 1.70 & 5.72 & 4EHV & 1.60 & 2.29 \\
\hline 2RG6 & 1.72 & 8.66 & 3KF7 & 2.00 & 7.40 & 4F9Y & 1.85 & 6.74 \\
\hline 2Y8O & 1.95 & 5.12 & 3L8S & 2.35 & 7.00 & 4KIN & 1.97 & 8.32 \\
\hline 2YIS & 2.00 & 8.77 & 3L8X & 2.15 & 8.00 & 4KIP & 2.27 & 8.15 \\
\hline 2YIW & 2.00 & 9.40 & 3MPT & 1.89 & 6.60 & 4KIQ & 2.50 & 7.64 \\
\hline 2YIX & 2.30 & 8.48 & 3MVM & 2.00 & 8.41 & 4L8M & 2.10 & 9.00 \\
\hline 2ZAZ & 1.80 & 6.40 & 3NNU & 2.40 & 7.32 & 4LOO & 1.95 & 8.15 \\
\hline 2ZB0 & 2.10 & 6.62 & 3NNV & 2.10 & 7.57 & 4LOP & 2.05 & 8.15 \\
\hline 3BV2 & 2.40 & 9.36 & 3NNW & 1.89 & 8.05 & 4LOQ & 2.32 & 8.15 \\
\hline 3BX5 & 2.40 & 8.46 & 3NNX & 2.28 & 7.96 & & & \\
\hline
\end{tabular}

Table S11. Basic information of all 102 targets in DUD-E used in our screening power test

\begin{tabular}{|c|c|c|c|c|}
\hline $\begin{array}{c}\text { Target } \\
\text { Name }^{\mathbf{a}}\end{array}$ & Description & Actives & $\begin{array}{c}\text { Decoy } \\
\text { Binders }\end{array}$ & $\begin{array}{c}\text { PDB } \\
\text { Code }^{\mathbf{b}}\end{array}$ \\
\hline AA2AR & Adenosine A2a receptor & 482 & 31550 & 3EML \\
\hline ABL1 & Tyrosine-protein kinase ABL & 182 & 10750 & 2HZI \\
\hline ACE & Angiotensin-converting enzyme & 282 & 16900 & 3BKL \\
\hline ACES & Acetylcholinesterase & 453 & 26250 & 1E66 \\
\hline ADA & Adenosine deaminase & 93 & 5450 & 2E1W \\
\hline ADA17 & ADAM17 & 532 & 35900 & 2OI0 \\
\hline ADRB1 & Beta-1 adrenergic receptor & 247 & 15850 & 2VT4 \\
\hline ADRB2 & Beta-2 adrenergic receptor & 231 & 15000 & 3NY8 \\
\hline AKT1 & Serine/threonine-protein kinase AKT & 293 & 16450 & 3CQW \\
\hline AKT2 & Serine/threonine-protein kinase AKT2 & 117 & 6900 & 3D0E \\
\hline ALDR & Aldose reductase & 159 & 9000 & 2HV5 \\
\hline AMPC & Beta-lactamase & 48 & 2850 & 1L2S \\
\hline ANDR & Androgen Receptor & 269 & 14350 & 2AM9 \\
\hline AOFB & Monoamine oxidase B & 283 & 6900 & 1S3B \\
\hline BACE1 & Beta-secretase 1 & 152 & 9950 & 3L5D \\
\hline BRAF & Serine/threonine-protein kinase B-raf & 492 & 31172 & 3D4Q \\
\hline CAH2 & Carbonic anhydrase II & 199 & 10700 & 2CNK \\
\hline CASP3 & Caspase-3 & 474 & 27850 & 1H00 \\
\hline CDK2 & Cyclin-dependent kinase 2 & 41 & 3850 & 3BWM \\
\hline COMT & Catechol O-methyltransferase & 120 & 7450 & 1R9O \\
\hline CP2C9 & Cytochrome P450 2C9 & 170 & 11800 & 3NXU \\
\hline CP3A4 & Cytochrome P450 3A4 & 166 & 12150 & 3KRJ \\
\hline CSF1R & Macrophage colony stimulating factor receptor & & \\
\hline
\end{tabular}




\begin{tabular}{|c|c|c|c|c|}
\hline CXCR4 & C-X-C chemokine receptor type 4 & 40 & 3406 & 3ODU \\
\hline DEF & Peptide deformylase & 102 & 5700 & 1LRU \\
\hline DHI1 & 11-beta-hydroxysteroid dehydrogenase 1 & 387 & 19350 & 3FRJ \\
\hline DPP4 & Dipeptidyl peptidase IV & 533 & 40950 & $2 \mathrm{I} 78$ \\
\hline DRD3 & Dopamine D3 receptor & 480 & 34050 & 3PBL \\
\hline DYR & Dihydrofolate reductase & 231 & 17200 & $3 \mathrm{NXO}$ \\
\hline EGFR & Epidermal growth factor receptor erbB1 & 542 & 35050 & 2RGP \\
\hline ESR1 & Estrogen receptor alpha & 383 & 20685 & $1 \mathrm{SJ} 0$ \\
\hline ESR2 & Estrogen receptor beta & 367 & 20199 & $2 \mathrm{FSZ}$ \\
\hline FA10 & Coagulation factor $\mathrm{X}$ & 537 & 28325 & 3KL6 \\
\hline FA7 & Coagulation factor VII & 114 & 6250 & $1 \mathrm{~W} 7 \mathrm{X}$ \\
\hline FABP4 & Fatty acid binding protein adipocyte & 47 & 2750 & 2NNQ \\
\hline FAK1 & Focal adhesion kinase 1 & 100 & 5350 & $3 \mathrm{BZ3}$ \\
\hline FGFR1 & Fibroblast growth factor receptor 1 & 139 & 8700 & $3 \mathrm{C} 4 \mathrm{~F}$ \\
\hline FKB1A & FK506-binding protein $1 \mathrm{~A}$ & 111 & 5800 & $1 \mathrm{~J} 4 \mathrm{H}$ \\
\hline FNTA & $\begin{array}{c}\text { Protein } \\
\text { farnesyltransferase/geranylgeranyltransferase type I } \\
\text { alpha subunit }\end{array}$ & 592 & 51500 & $3 \mathrm{E} 37$ \\
\hline FPPS & Farnesyl diphosphate synthase & 85 & 8850 & 1ZW5 \\
\hline GCR & Glucocorticoid receptor & 258 & 15000 & 3BQD \\
\hline GLCM & Beta-glucocerebrosidase & 54 & 3800 & $2 \mathrm{~V} 3 \mathrm{~F}$ \\
\hline GRIA2 & Glutamate receptor ionotropic, AMPA 2 & 158 & 11845 & $3 \mathrm{KGC}$ \\
\hline GRIK1 & Glutamate receptor ionotropic kainate 1 & 101 & 6550 & $1 \mathrm{VSO}$ \\
\hline HDAC2 & Histone deacetylase 2 & 185 & 10300 & $3 \mathrm{MAX}$ \\
\hline HDAC8 & Histone deacetylase 8 & 170 & 10450 & $3 \mathrm{~F} 07$ \\
\hline HIVINT & Human immunodeficiency virus type 1 integrase & 100 & 6650 & 3NF7 \\
\hline HIVPR & Human immunodeficiency virus type 1 protease & 536 & 35750 & 1XL2 \\
\hline HIVRT & $\begin{array}{l}\text { Human immunodeficiency virus type } 1 \text { reverse } \\
\text { transcriptase }\end{array}$ & 338 & 18891 & $3 \mathrm{LAN}$ \\
\hline HMDH & HMG-CoA reductase & 170 & 8750 & $3 \mathrm{CCW}$ \\
\hline HS90A & Heat shock protein HSP 90-alpha & 88 & 4850 & $1 \mathrm{UYG}$ \\
\hline HXK4 & Hexokinase type IV & 92 & 4700 & 3F9M \\
\hline IGF1R & Insulin-like growth factor I receptor & 148 & 9300 & $20 J 9$ \\
\hline INHA & Enoyl-[acyl-carrier-protein] reductase & 44 & 2300 & $2 \mathrm{H} 7 \mathrm{~L}$ \\
\hline ITAL & Leukocyte adhesion glycoprotein LFA-1 alpha & 138 & 8500 & 2ICA \\
\hline JAK2 & Tyrosine-protein kinase JAK2 & 130 & 6500 & $3 \mathrm{LPB}$ \\
\hline KIF11 & Kinesin-like protein 1 & 116 & 6850 & $3 \mathrm{CJO}$ \\
\hline KIT & Stem cell growth factor receptor & 166 & 10450 & $3 \mathrm{G} 0 \mathrm{E}$ \\
\hline KITH & Thymidine kinase & 57 & 2850 & $2 \mathrm{~B} 8 \mathrm{~T}$ \\
\hline $\mathrm{KPCB}$ & Protein kinase $\mathrm{C}$ beta & 135 & 8700 & $2 \mathrm{I} 0 \mathrm{E}$ \\
\hline LCK & Tyrosine-protein kinase LCK & 420 & 27400 & $2 \mathrm{OF} 2$ \\
\hline LKHA4 & Leukotriene A4 hydrolase & 171 & 9450 & $3 \mathrm{CHP}$ \\
\hline MAPK2 & MAP kinase-activated protein kinase 2 & 101 & 6150 & $3 \mathrm{M} 2 \mathrm{~W}$ \\
\hline MCR & Mineralocorticoid receptor & 94 & 5150 & 2AA2 \\
\hline MET & Hepatocyte growth factor receptor & 166 & 11250 & 3LQ8 \\
\hline MK01 & MAP kinase ERK2 & 79 & 4550 & $2 \mathrm{OJG}$ \\
\hline MK10 & c-Jun N-terminal kinase 3 & 104 & 6600 & 2ZDT \\
\hline MK14 & MAP kinase p38 alpha & 578 & 35850 & 2QD9 \\
\hline MMP13 & Matrix metalloproteinase 13 & 572 & 37200 & $830 \mathrm{C}$ \\
\hline MP2K1 & $\begin{array}{l}\text { Dual specificity mitogen-activated protein kinase } \\
\text { kinase } 1\end{array}$ & 121 & 8150 & $3 \mathrm{EQH}$ \\
\hline NOS1 & Nitric-oxide synthase, brain & 100 & 8050 & 1QW6 \\
\hline NRAM & Neuraminidase & 98 & 6200 & $1 \mathrm{~B} 9 \mathrm{~V}$ \\
\hline PA2GA & Phospholipase A2 group IIA & 99 & 5150 & $1 \mathrm{KVO}$ \\
\hline PARP1 & Poly [ADP-ribose] polymerase-1 & 508 & 30050 & $3 \mathrm{~L} 3 \mathrm{M}$ \\
\hline
\end{tabular}




\begin{tabular}{|c|c|c|c|c|}
\hline PDE5A & Phosphodiesterase 5A & 398 & 27550 & 1UDT \\
\hline PGH1 & Cyclooxygenase-1 & 195 & 10800 & 2OYU \\
\hline PGH2 & Cyclooxygenase-2 & 435 & 23150 & 3LN1 \\
\hline PLK1 & Serine/threonine-protein kinase PLK1 & 107 & 6800 & 2OWB \\
\hline PNPH & Purine nucleoside phosphorylase & 103 & 6950 & 3BGS \\
\hline PPARA & Peroxisome proliferator-activated receptor alpha & 373 & 19399 & 2P54 \\
\hline PPARD & Peroxisome proliferator-activated receptor delta & 240 & 12250 & 2ZNP \\
\hline PPARG & Peroxisome proliferator-activated receptor gamma & 484 & 25300 & 2GTK \\
\hline PRGR & Progesterone receptor & 293 & 15650 & 3KBA \\
\hline PTN1 & Protein-tyrosine phosphatase 1B & 130 & 7250 & 2AZR \\
\hline PUR2 & GAR transformylase & 50 & 2700 & 1NJS \\
\hline PYGM & Muscle glycogen phosphorylase & 77 & 3950 & 1C8K \\
\hline PYRD & Dihydroorotate dehydrogenase & 111 & 6450 & 1D3G \\
\hline RENI & Renin & 104 & 6958 & 3G6Z \\
\hline ROCK1 & Rho-associated protein kinase 1 & 100 & 6300 & 2ETR \\
\hline RXRA & Retinoid X receptor alpha & 131 & 6950 & 1MV9 \\
\hline SAHH & Adenosylhomocysteinase & 63 & 3450 & 1LI4 \\
\hline SRC & Tyrosine-protein kinase SRC & 524 & 34500 & 3EL8 \\
\hline TGFR1 & TGF-beta receptor type I & 133 & 8500 & 3HMM \\
\hline THB & Thyroid hormone receptor beta-1 & 103 & 7450 & 1Q4X \\
\hline THRB & Thrombin & 461 & 27004 & 1YPE \\
\hline TRY1 & Trypsin I & 449 & 25980 & 2AYW \\
\hline TRYB1 & Tryptase beta-1 & 148 & 7650 & 2ZEC \\
\hline TYSY & Thymidylate synthase & 109 & 6750 & 1SYN \\
\hline UROK & Urokinase-type plasminogen activator & 162 & 9850 & 1SQT \\
\hline VGFR2 & Vascular endothelial growth factor receptor 2 & 409 & 24950 & 2P2I \\
\hline WEE1 & Serine/threonine-protein kinase WEE1 & 102 & 6150 & 3BIZ \\
\hline XIAP & Inhibitor of apoptosis protein 3 & 100 & 5150 & 3HL5 \\
\hline
\end{tabular}

a: Target name provided in DUD-E database (http://dude.docking.org/targets), where the actives and decoys were defined according to the standards used by DUD-E.

${ }^{\mathrm{b}}$ : The PDB entry from which the protein structure was retrieved and employed in virtual screening trials. 


\section{PART 3. Integration of desolvation energies by water displacement into}

\section{scoring functions}

\section{Calculation of Desolvation Energy by WatEr Displacement (DEWED)}

According to the classical theory, the free energy change in a protein-ligand binding process can be dissected as the sum of $\Delta G_{b i n d, g a s,} \Delta G_{\text {desolv }}^{\text {prot }}$ and $\Delta G_{\text {desolv }}^{\text {lig }}$ With the hydration sites predicted by HydraMap, we can compute $\Delta G_{\text {desolv }}^{\text {prot }}$ in an explicit manner. The basic concept of our method is illustrated in Figure S4. Before the protein-ligand complex is formed, the binding pocket is filled with water molecules, which can be deduced by HydraMap. After the complex is formed, some water molecules inside the binding pocket are driven out to the bulk solvent. Our method superimposes all hydration sites with the bound ligand to determine which sites are occupied upon ligand binding. Then, the energy change associated with the displacement of water molecules on those occupied hydration sites is computed, which yields $\Delta G_{\text {desolv }}^{\text {prot }}$ Here, we assume that the other parts of the solvation shell of the protein molecule that are not directly affected by ligand binding do not contribute to $\Delta G_{d e s o l v}^{\text {prot }}$.
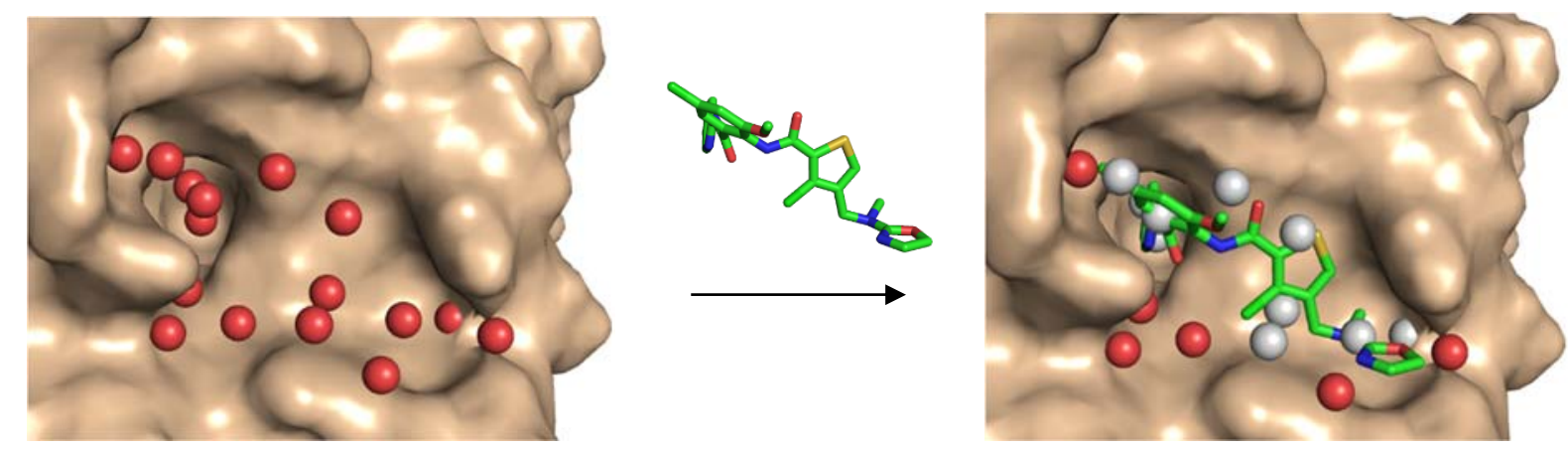

Figure S4. Illustration of the desolvation effect originated at the protein side upon ligand binding. Hydration sites inside the binding pocket, i.e. energetically favorable locations for hosting water molecules, are represented by red spheres. The white spheres on the right figure represent the hydration sites displaced by the ligand after the complex is formed.

The computation of the desolvation energy from the protein side $\left(\Delta G_{\text {desolv }}^{\text {prot }}\right)$, was inspired by the displaced-solvent algorithm developed by Friesner et al. ${ }^{1}$ The formula implemented in our study is as follows: 


$$
\Delta G_{\text {desolv }}^{\text {prot }}=\sum_{i}\left[\Delta G_{h s, i} \times W_{h s, i}\right]=\sum_{i}\left[\left(G_{h s, i}-G_{b u l k}\right) \times W_{h s, i}\right]
$$

In Eq. S1, $\Delta G_{d e s o l v}^{\text {prot }}$ is the total free energy change of the water molecules inside and around the binding pocket that are displaced by the ligand molecule upon binding. Conceptually, it is computed by summing up the contribution from each relevant hydration site. The contribution of the ith hydration site $\left(\Delta G_{h s, i}\right)$ is computed as the difference between the PMF energy of a water molecule at this site $\left(G_{h s, i}\right)$ and the PMF energy of a water molecule in the bulk solvent $\left(G_{b u l k}\right)$. The former is calculated as the PMF energy of the hydration site $i$ from the interactions with the protein atoms and other hydration sites nearby. To compute $G_{b u l k}$, a cubic TIP3P water box with its length as $20 \AA$ was equilibrated via an MD simulation of $10 \mathrm{~ns}$ long at $300 \mathrm{~K}$ and the constant pressure of $1 \mathrm{~atm}$. The average PMF energy of all water molecules in the box derived from the last snapshot was calculated to be $-3.84 \mathrm{kcal} / \mathrm{mol}$, which was used as the value of $G_{b u l k}$ in this study.

Note that in Eq. S1, the contribution of the ith hydration site is modified by a weight factor $W_{h s, i}$. This weight factor gives a penalty to $\Delta G_{h s, I}$ due to the deviation from an ideal scenario, which is computed as:

$$
W_{h s, i}=\left(1-\frac{d_{m i n, i}}{d_{0}}\right) \times I F\left(d_{0}-d_{m i n, i}\right) \times I F\left(G_{0}-G_{h s, i}\right)
$$

Here, $d_{\min , i}$ is the minimal distance between this hydration site and any heavy atom in the ligand molecule. If it is shorter than a user-defined cutoff $\left(d_{0}\right)$, the indicator function $(I F)$ is one; otherwise, it is zero. This ensures that this hydration site is effectively occupied by the bound ligand. No penalty is given to this aspect if this hydration site overlaps perfectly with a certain ligand atom (i.e. $\left.d_{\min , i}=0\right)$ ). $G_{0}$ is a user-defined cutoff. When the PMF energy of a hydration site is lower than this value, it will be considered in the desolvation energy calculation. Both $d_{0}$ and $G_{0}$ are adjustable parameters, which will be optimized in the subsequent process.

\section{Multi-objective optimization of the derivative scoring functions}

Two essential aspects of a scoring function, namely "scoring power" and "docking power", were considered in the optimization of the derivative scoring functions. In general, a scoring function will be more useful in practice if it is good at both aspects. Thus, we adopted a multiobjective optimization strategy to achieve a balanced performance between scoring power and docking power for each scoring function. The target function (TF) used in this optimization 
process is the product of two quantitative indicators, where DP and $S P$ are the indicators for docking power and scoring power, respectively (Eq. S3):

$$
\begin{aligned}
& T F=D P \times S P \\
& D P=\frac{\sum_{i=1}^{N} S_{i}}{N} \\
& S P=\frac{\sum_{i=1}^{N}\left(E x p_{i}-\langle E x p\rangle\right) \times\left(\operatorname{Comp}_{i}-\langle\operatorname{Comp}\rangle\right)}{\sqrt{\sum_{i=1}^{N}\left(E x p_{i}-\langle E x p\rangle\right)^{2}} \sqrt{\sum_{i=1}^{N}\left(\operatorname{Comp}_{i}-\langle\operatorname{Comp}\rangle\right)^{2}}}
\end{aligned}
$$

The docking power $(D P)$ of a given scoring function was computed as its success rate of identifying the native ligand binding poses on a data set of $N$ protein-ligand complexes (Eq. S4). For each complex included in this data set, a certain number of decoy ligand binding poses must be generated in prior. For this task, we adopted the procedure employed previously in our CASF-2013 benchmark. ${ }^{2}$ In brief, three types of molecular docking software (GOLD, Surflex, and MOE) were employed to generate the possible ligand binding poses for each complex in the test set. Their outputs were combined and clustered. Then, a total of up to 100 representative ligand binding poses were selected. The given scoring function was applied to compute the binding scores of all ligand binding poses for this complex. If the RMSD value between the native ligand binding pose and the top-ranked binding pose selected by the scoring function is below a pre-set cutoff (e.g. $2.0 \AA$ ), one point is rewarded to the given scoring function (i.e. $S_{i}=$ 1); otherwise, no point is gained (i.e. $S_{i}=0$ ). The overall success rate can be computed on the entire data set, which is a value between 0 and 1 .

The scoring power (SP) of the scoring function under consideration is computed as the Pearson correlation coefficient between the experimental binding data, and the binding scores produced by the scoring function for the $N$ protein-ligand complexes in a data set (Eq. S5). Here, $\operatorname{Exp}_{i}$ denotes for the experimental data (in $\log K_{\mathrm{a}}$ units) of the $i$ th complex in the data set; while $<E x p>$ is the mean value over the entire data set. Compi denotes for the computed binding score of the ith complex in the data set; while $<$ Comp $>$ is the mean value over the entire data set. As a Pearson correlation coefficient, the scoring power $(S P)$ is a value between 0 and 1.

With the definition of the target function in Equation S3, the optimization process for the derivative scoring functions (Eqs. 6-9 in the main text) aimed at maximizing the target function by adjusting the weight factors before the energy terms in each scoring function (i.e. $C_{0} \sim C_{n}$ ). The optimization process can be divided into two major steps. At the first step, the optimal 
parameters included in the desolvation energy term $\left(\Delta G_{\text {desolv }}^{\text {prot }}\right)$ were determined. As indicated in Eq. S2, the cutoff values $G_{0}$ and $d_{0}$ will affect the final outcome of this term. To determine the optimal setting of these parameters, Go was varied from -2.5 to -0.5 with an increment of 0.1 , and $d_{0}$ was varied from $2.2 \AA$ to $2.8 \AA$ with an increment of $0.1 \AA$. Our preliminary trials on the multiple linear regression (MLR) analysis showed the change of $d_{0}$ had a very small effect on the $\mathrm{R}_{\mathrm{p}}$ values. Thus, we set $d_{0}$ as the median, $2.5 \AA$ in this step so as to decrease the optimization complexity.

Given a $G_{0}$ value, the desolvation energies could be calculated as Equation S1. MLR analysis was performed to derive the initial weight factors $\left(C_{0} \sim C_{n}\right)$ for each energy term in the derivative scoring functions. A steady-state Genetic Algorithm (GA) process ${ }^{3}$ was adopted to optimize these weight factors by maximizing the target function (Eq. S3). The weight factors were allowed to vary between one-tenth and 10-fold of the initial values. A total of 100 combinations of weight factors (i.e. 100 chromosomes) was generated randomly as the initial population. Both the mutation rate and the cross-over rate were set to 0.5 . A total of $10,000 \mathrm{GA}$ steps were conducted for each optimization job. After optimization, the average docking power, scoring power provided in 100 chromosomes were used as the final results. Comparing the optimization results obtained from different $G_{0}$ values, the final optimal docking power, scoring power, and optimized weight factors were determined. 
PART 4. Comparisons of the performance of original scoring functions and their derivative scoring functions with additional desolvation terms

Table S12. Summary of the scoring power test and docking power test of the original and the derivative scoring functions calibrated on the PDBbind core set and five target-specific data sets

\begin{tabular}{|c|c|c|c|c|c|c|c|}
\hline \multirow[t]{2}{*}{ Data Set $^{\mathbf{a}}$} & \multirow{2}{*}{$\begin{array}{l}\text { Scoring } \\
\text { function }^{b}\end{array}$} & \multicolumn{3}{|c|}{ Scoring Power $\left(R_{p}\right)^{c}$} & \multicolumn{3}{|c|}{$\begin{array}{c}\text { Docking Power }{ }^{\mathrm{d}} \text { (success } \\
\text { rate } \% \text { ) }\end{array}$} \\
\hline & & Original $^{\mathrm{e}}$ & DEWED $^{f}$ & $\mathrm{~GB} / \mathrm{SA}^{\mathrm{g}}$ & Original & DEWED & $\mathrm{GB} / \mathrm{SA}$ \\
\hline \multirow{4}{*}{$\begin{array}{l}\text { PDBbind } \\
\text { core set as } \\
\text { training set } \\
(280)^{\mathrm{h}}\end{array}$} & ASP & 0.631 & 0.662 & 0.640 & 82.5 & 83.2 & 79.7 \\
\hline & ChemPLP & 0.603 & 0.636 & 0.632 & 85.3 & 85.6 & 85.9 \\
\hline & GoldScore & 0.499 & 0.657 & 0.665 & 74.4 & 74.5 & 72.6 \\
\hline & X-Score & 0.631 & 0.624 & 0.623 & 62.5 & 73.9 & 72.8 \\
\hline \multirow{4}{*}{$\begin{array}{l}\text { PDBbind } \\
\text { core set as } \\
\text { test set }(5)^{\mathrm{h}}\end{array}$} & ASP & 0.707 & 0.700 & 0.697 & 82.5 & 78.2 & 76.8 \\
\hline & ChemPLP & 0.729 & 0.714 & 0.722 & 85.3 & 83.9 & 82.8 \\
\hline & GoldScore & 0.650 & 0.737 & 0.733 & 74.4 & 69.8 & 70.5 \\
\hline & X-Score & 0.717 & 0.732 & 0.745 & 62.5 & 70.2 & 71.6 \\
\hline \multirow{4}{*}{$\begin{array}{c}\text { AchE } \\
\text { Training set } \\
\text { (39) }\end{array}$} & ASP & 0.782 & 0.784 & 0.803 & 56.4 & 66.7 & 66.7 \\
\hline & ChemPLP & 0.785 & 0.816 & 0.825 & 64.1 & 76.9 & 74.4 \\
\hline & GoldScore & 0.520 & 0.820 & 0.799 & 43.6 & 64.1 & 59.0 \\
\hline & X-Score & 0.844 & 0.874 & 0.820 & 53.8 & 53.9 & 48.7 \\
\hline \multirow{4}{*}{$\begin{array}{c}\text { BACE-1 } \\
\text { Training set } \\
\quad(161)\end{array}$} & ASP & 0.692 & 0.718 & 0.702 & 83.9 & 91.3 & 88.8 \\
\hline & ChemPLP & 0.693 & 0.739 & 0.729 & 92.5 & 96.3 & 94.4 \\
\hline & GoldScore & 0.533 & 0.758 & 0.729 & 87.6 & 88.2 & 88.2 \\
\hline & X-Score & 0.713 & 0.762 & 0.746 & 77.6 & 86.3 & 82.0 \\
\hline \multirow{4}{*}{$\begin{array}{c}\text { BACE-1 } \\
\text { Test set } \\
(57)\end{array}$} & ASP & 0.658 & 0.704 & 0.692 & 78.9 & 87.7 & 84.2 \\
\hline & ChemPLP & 0.701 & 0.743 & 0.737 & 91.2 & 94.7 & 91.2 \\
\hline & GoldScore & 0.606 & 0.730 & 0.706 & 84.2 & 82.5 & 82.5 \\
\hline & X-Score & 0.689 & 0.759 & 0.743 & 84.2 & 86.0 & 84.2 \\
\hline \multirow{4}{*}{$\begin{array}{c}\text { FXa } \\
\text { Training set } \\
(79)\end{array}$} & ASP & 0.100 & 0.230 & 0.227 & 92.4 & 95.0 & 91.1 \\
\hline & ChemPLP & 0.234 & 0.324 & 0.317 & 100 & 100 & 100 \\
\hline & GoldScore & 0.105 & 0.253 & 0.261 & 92.4 & 96.9 & 91.5 \\
\hline & X-Score & 0.436 & 0.352 & 0.347 & 87.3 & 94.9 & 93.7 \\
\hline \multirow{4}{*}{$\begin{array}{c}\text { HIV-1 } \\
\text { protease } \\
\text { training set } \\
\text { (129) }\end{array}$} & ASP & 0.048 & 0.045 & 0.318 & 98.4 & 96.1 & 91.5 \\
\hline & ChemPLP & 0.087 & 0.198 & 0.355 & 89.1 & 85.3 & 84.5 \\
\hline & GoldScore & 0.176 & 0.283 & 0.421 & 79.1 & 77.5 & 69.8 \\
\hline & X-Score & 0.231 & 0.162 & 0.335 & 68.2 & 75.2 & 69.0 \\
\hline \multirow{3}{*}{$\begin{array}{c}\text { HIV-1 } \\
\text { protease } \\
\text { Test set }\end{array}$} & ASP & 0.154 & 0.190 & 0.061 & 95.3 & 95.3 & 81.4 \\
\hline & ChemPLP & 0.128 & 0.020 & 0.162 & 86.0 & 81.4 & 76.7 \\
\hline & GoldScore & 0.211 & 0.158 & 0.284 & 69.8 & 81.4 & 69.8 \\
\hline
\end{tabular}




\begin{tabular}{|c|c|c|c|c|c|c|c|}
\hline$(43)$ & X-Score & 0.049 & 0.045 & 0.110 & 60.5 & 81.4 & 69.8 \\
\hline \multirow{3}{*}{$\begin{array}{c}\text { MAPK14 } \\
\text { Training set } \\
(87)\end{array}$} & ASP & 0.564 & 0.566 & 0.563 & 93.1 & 96.6 & 96.6 \\
\cline { 2 - 8 } & ChemPLP & 0.517 & 0.550 & 0.541 & 96.6 & 96.6 & 98.9 \\
\cline { 2 - 8 } & GoldScore & 0.516 & 0.552 & 0.542 & 88.5 & 89.7 & 88.5 \\
\cline { 2 - 8 } & X-Score & 0.499 & 0.537 & 0.528 & 82.8 & 92.0 & 93.1 \\
\hline \multirow{3}{*}{$\begin{array}{c}\text { MAPK14 } \\
\text { Test set }\end{array}$} & ASP & 0.672 & 0.699 & 0.689 & 93.1 & 93.1 & 93.1 \\
\cline { 2 - 8 } & ChemPLP & 0.634 & 0.659 & 0.635 & 93.1 & 89.7 & 89.7 \\
\cline { 2 - 8 } & GoldScore & 0.593 & 0.640 & 0.639 & 89.7 & 89.7 & 93.1 \\
\cline { 2 - 8 } & X-Score & 0.642 & 0.679 & 0.611 & 82.8 & 89.7 & 93.1 \\
\hline
\end{tabular}

${ }^{a}$ The number within the brackets is the total number of protein-ligand complexes in this data set.

${ }^{\mathrm{b}}$ Four general scoring functions ASP, ChemPLP, GoldScore and X-Score are used in this research.

${ }^{\mathrm{c}}$ Scoring power is measured as the Pearson correlation coefficient $\left(R_{\mathrm{p}}\right)$ in fitting the experimental binding data and computed binding scores.

${ }^{\mathrm{d}}$ Docking power is measured as the success rate (\%) of identifying the native ligand binding pose with consideration of the best-scored docking pose.

${ }^{\mathrm{e}}$ Results for original scoring functions.

${ }^{\mathrm{f}}$ Results for the scoring model optimized with the energy terms provided by original scoring functions and the DEWED desolvation energy term.

${ }^{g}$ Results for the scoring model optimized with the energy terms provided by original scoring functions and the GB/SA desolvation energy term.

${ }^{\mathrm{h}}$ The scoring power and docking power given here are the average results produced by 57 individual models. 
Table S13. Outcomes of the screening power test for the original and the derivative scoring functions calibrated on the PDBbind core set and five target-specific data sets

\begin{tabular}{|c|c|c|c|c|c|c|c|}
\hline \multirow[t]{2}{*}{ Target } & \multirow{2}{*}{$\begin{array}{l}\text { Scoring } \\
\text { Function }^{\mathrm{a}}\end{array}$} & \multicolumn{3}{|c|}{$\mathbf{E F}_{0.2 \%}{ }^{\mathbf{b}}$} & \multicolumn{3}{|c|}{$\mathbf{E F}_{1 \%}$} \\
\hline & & Original $^{\mathfrak{c}}$ & $\begin{array}{c}\text { DEWED- } \\
\mathbf{S}^{\mathbf{d}}\end{array}$ & $\begin{array}{l}\text { DEWED- } \\
\mathbf{G}^{\mathrm{e}}\end{array}$ & Original & $\begin{array}{c}\text { DEWED- } \\
\mathrm{S}\end{array}$ & $\begin{array}{l}\text { DEWED- } \\
\text { G }\end{array}$ \\
\hline \multirow[t]{4}{*}{ AchE } & ASP & 41.94 & 41.94 & 49.67 & 21.41 & 20.09 & 20.31 \\
\hline & ChemPLP & 43.05 & 56.29 & 39.74 & 19.87 & 28.26 & 14.35 \\
\hline & GoldScore & 1.10 & 23.18 & 24.28 & 1.32 & 8.83 & 9.27 \\
\hline & X-Score & 5.52 & 8.83 & 13.25 & 4.42 & 2.87 & 6.18 \\
\hline \multirow[t]{4}{*}{ BACE } & ASP & 12.59 & 23.38 & 19.78 & 10.43 & 10.07 & 14.39 \\
\hline & ChemPLP & 12.59 & 16.19 & 16.19 & 7.55 & 12.95 & 10.07 \\
\hline & GoldScore & 10.79 & 23.38 & 5.40 & 5.40 & 7.55 & 4.68 \\
\hline & X-Score & 8.99 & 5.40 & 21.58 & 3.96 & 4.68 & 9.35 \\
\hline \multirow[t]{4}{*}{ FXa } & ASP & 48.42 & 46.55 & 47.49 & 26.07 & 25.88 & 28.12 \\
\hline & ChemPLP & 31.66 & 20.48 & 20.48 & 17.69 & 12.85 & 13.97 \\
\hline & GoldScore & 22.35 & 27.93 & 29.80 & 12.10 & 15.83 & 16.76 \\
\hline & X-Score & 37.24 & 43.76 & 41.90 & 20.48 & 29.61 & 27.37 \\
\hline \multirow[t]{4}{*}{ HIV-1 } & ASP & 6.53 & 19.59 & 23.32 & 3.92 & 12.13 & 8.96 \\
\hline & ChemPLP & 3.73 & 4.66 & 9.33 & 2.99 & 2.43 & 5.41 \\
\hline & GoldScore & 0.00 & 0.00 & 2.80 & 0.00 & 0.37 & 1.87 \\
\hline & X-Score & 8.40 & 13.99 & 13.99 & 5.78 & 9.33 & 12.87 \\
\hline \multirow[t]{4}{*}{ MAPK14 } & ASP & 15.57 & 19.03 & 24.22 & 10.21 & 12.80 & 12.28 \\
\hline & ChemPLP & 13.84 & 21.63 & 21.63 & 7.61 & 10.38 & 9.86 \\
\hline & GoldScore & 0.87 & 7.79 & 3.46 & 2.25 & 4.33 & 3.11 \\
\hline & X-Score & 2.60 & 20.76 & 15.57 & 3.46 & 15.22 & 11.76 \\
\hline
\end{tabular}

${ }^{\text {a }}$ Four general scoring functions ASP, ChemPLP, GoldScore and X-Score are used in this research.

${ }^{\mathrm{b}}$ Screening power is measured as the enrichment factors when the top $0.2 \%$ and top $1 \%$ of the compounds are considered.

${ }^{\mathrm{c}}$ Results for original models of four scoring functions.

${ }^{\mathrm{d}}$ Results for scoring models optimized with the energy terms provided by the four scoring functions and the desolvation energy term, on the date sets for the specific targets.

${ }^{\mathrm{e}}$ Results for scoring models optimized with the energy terms provided by the four scoring functions and the desolvation energy term, on PDBbind core set. 
Table S14. Outcomes of the screening power test for X-Score and X-Score ${ }^{\text {DEWED }}$ (calibrated on the PDBbind core set) on all 102 targets in the DUD-E benchmark

\begin{tabular}{|c|c|c|c|c|c|c|}
\hline \multirow[t]{2}{*}{ Target } & \multicolumn{2}{|r|}{$\mathbf{E F}_{\mathbf{0 . 2} \%}$} & \multicolumn{2}{|r|}{$\mathbf{E F}_{1 \%}$} & \multicolumn{2}{|r|}{ AUC } \\
\hline & X-Score & X-Score $^{\text {DEWED-G }}$ & X-Score & X-Score ${ }^{\text {DEWED-G }}$ & X-Score & X-Score ${ }^{\text {DEWED-G }}$ \\
\hline AA2AR & 3.11 & 6.22 & 1.24 & 2.70 & 0.521 & 0.575 \\
\hline $\mathrm{ABL}$ & 0.00 & 27.47 & 1.65 & 14.84 & 0.556 & 0.717 \\
\hline $\mathrm{ACE}$ & 26.60 & 15.96 & 12.41 & 6.38 & 0.717 & 0.637 \\
\hline ACES & 5.52 & 13.25 & 4.42 & 6.18 & 0.611 & 0.623 \\
\hline ADA & 0.00 & 0.00 & 0.00 & 1.08 & 0.504 & 0.591 \\
\hline ADA17 & 13.16 & 15.04 & 8.27 & 10.90 & 0.677 & 0.722 \\
\hline ADRB1 & 2.02 & 16.19 & 2.43 & 6.07 & 0.611 & 0.631 \\
\hline ADRB2 & 2.16 & 17.32 & 1.30 & 9.09 & 0.615 & 0.640 \\
\hline AKT1 & 3.41 & 3.41 & 3.07 & 3.07 & 0.640 & 0.719 \\
\hline AKT2 & 0.00 & 34.19 & 4.27 & 19.66 & 0.720 & 0.768 \\
\hline ALDR & 3.14 & 6.29 & 0.63 & 2.52 & 0.548 & 0.576 \\
\hline AMPC & 0.00 & 0.00 & 2.08 & 4.17 & 0.617 & 0.572 \\
\hline ANDR & 5.58 & 7.43 & 2.60 & 3.72 & 0.645 & 0.717 \\
\hline AOFB & 0.00 & 0.00 & 2.46 & 0.00 & 0.580 & 0.603 \\
\hline BACE1 & 8.99 & 21.58 & 3.96 & 9.35 & 0.694 & 0.756 \\
\hline BRAF & 13.16 & 19.74 & 3.29 & 10.53 & 0.730 & 0.778 \\
\hline CAH2 & 1.02 & 1.02 & 2.24 & 1.22 & 0.586 & 0.577 \\
\hline CASP3 & 15.08 & 20.10 & 7.04 & 12.06 & 0.697 & 0.666 \\
\hline CDK2 & 0.00 & 12.66 & 2.74 & 6.75 & 0.631 & 0.677 \\
\hline COMT & 0.00 & 0.00 & 0.00 & 0.00 & 0.547 & 0.609 \\
\hline CP2C9 & 16.67 & 20.83 & 7.50 & 6.67 & 0.593 & 0.590 \\
\hline CP3A4 & 0.00 & 14.71 & 2.35 & 7.65 & 0.565 & 0.623 \\
\hline CSF1R & 3.01 & 24.10 & 4.82 & 12.05 & 0.716 & 0.811 \\
\hline CXCR4 & 0.00 & 25.00 & 7.50 & 5.00 & 0.708 & 0.751 \\
\hline DEF & 4.90 & 14.71 & 12.75 & 11.76 & 0.738 & 0.731 \\
\hline DHI1 & 0.00 & 3.03 & 2.42 & 1.82 & 0.670 & 0.616 \\
\hline DPP4 & 0.00 & 4.69 & 0.00 & 3.75 & 0.592 & 0.676 \\
\hline DRD3 & 0.00 & 1.04 & 0.21 & 1.67 & 0.535 & 0.587 \\
\hline DYR & 2.16 & 12.99 & 3.03 & 4.76 & 0.683 & 0.739 \\
\hline EGFR & 1.85 & 28.60 & 1.66 & 12.92 & 0.506 & 0.607 \\
\hline ESR1 & 13.09 & 31.41 & 7.59 & 14.92 & 0.731 & 0.726 \\
\hline ESR2 & 2.73 & 21.86 & 4.92 & 13.93 & 0.714 & 0.740 \\
\hline FA10 & 37.24 & 41.90 & 20.48 & 27.37 & 0.798 & 0.801 \\
\hline FA7 & 26.32 & 35.09 & 12.28 & 25.44 & 0.837 & 0.827 \\
\hline FABP4 & 10.64 & 10.64 & 14.89 & 8.51 & 0.612 & 0.601 \\
\hline FAK1 & 0.00 & 20.00 & 1.00 & 12.00 & 0.604 & 0.812 \\
\hline FGFR1 & 0.00 & 3.60 & 2.16 & 6.47 & 0.597 & 0.740 \\
\hline FKB1A & 0.00 & 22.52 & 5.41 & 12.61 & 0.809 & 0.745 \\
\hline FNTA & 2.53 & 7.60 & 2.87 & 5.74 & 0.719 & 0.711 \\
\hline FPPS & 0.00 & 0.00 & 0.00 & 0.00 & 0.722 & 0.709 \\
\hline GCR & 3.88 & 13.57 & 1.16 & 6.98 & 0.679 & 0.691 \\
\hline GLCM & 0.00 & 0.00 & 0.00 & 1.85 & 0.587 & 0.570 \\
\hline GRIA2 & 0.00 & 12.66 & 1.27 & 5.70 & 0.650 & 0.716 \\
\hline GRIK1 & 0.00 & 4.95 & 0.00 & 3.96 & 0.635 & 0.644 \\
\hline HDAC2 & 8.11 & 13.51 & 5.41 & 8.11 & 0.718 & 0.718 \\
\hline HDAC8 & 0.00 & 0.00 & 1.76 & 0.59 & 0.721 & 0.720 \\
\hline HIVINT & 0.00 & 0.00 & 4.00 & 3.00 & 0.598 & 0.667 \\
\hline HIVPR & 8.40 & 13.99 & 5.78 & 12.87 & 0.778 & 0.753 \\
\hline HIVRT & 0.00 & 0.00 & 0.30 & 0.89 & 0.507 & 0.579 \\
\hline
\end{tabular}




\begin{tabular}{|c|c|c|c|c|c|c|}
\hline HMDH & 2.94 & 38.24 & 5.29 & 18.82 & 0.836 & 0.823 \\
\hline HS90A & 0.00 & 0.00 & 0.00 & 0.00 & 0.783 & 0.674 \\
\hline HXK4 & 0.00 & 0.00 & 0.00 & 1.09 & 0.710 & 0.771 \\
\hline IGF1R & 0.00 & 23.65 & 2.70 & 12.84 & 0.650 & 0.782 \\
\hline INHA & 34.88 & 11.63 & 11.63 & 6.98 & 0.579 & 0.587 \\
\hline ITAL & 0.00 & 0.00 & 0.00 & 0.72 & 0.514 & 0.549 \\
\hline JAK2 & 18.69 & 46.73 & 7.48 & 17.76 & 0.685 & 0.771 \\
\hline KIF11 & 8.62 & 21.55 & 6.90 & 19.83 & 0.860 & 0.855 \\
\hline KIT & 15.06 & 30.12 & 7.23 & 13.25 & 0.728 & 0.800 \\
\hline KITH & 0.00 & 52.63 & 15.79 & 31.58 & 0.684 & 0.728 \\
\hline KPCB & 11.11 & 37.04 & 9.63 & 25.19 & 0.748 & 0.799 \\
\hline LCK & 5.95 & 45.24 & 6.43 & 19.05 & 0.695 & 0.797 \\
\hline LKHA4 & 5.85 & 26.32 & 4.68 & 11.70 & 0.785 & 0.785 \\
\hline MAPK2 & 0.00 & 9.90 & 4.95 & 1.98 & 0.753 & 0.788 \\
\hline MCR & 0.00 & 0.00 & 0.00 & 1.06 & 0.692 & 0.740 \\
\hline MET & 0.00 & 66.27 & 3.61 & 43.37 & 0.727 & 0.836 \\
\hline MK01 & 0.00 & 6.33 & 1.27 & 2.53 & 0.692 & 0.767 \\
\hline MK10 & 4.81 & 14.42 & 5.77 & 8.65 & 0.623 & 0.699 \\
\hline MK14 & 2.60 & 15.57 & 3.46 & 11.76 & 0.725 & 0.791 \\
\hline MMP13 & 12.24 & 29.72 & 9.27 & 12.76 & 0.687 & 0.724 \\
\hline MP2K1 & 0.00 & 4.13 & 2.48 & 1.65 & 0.508 & 0.618 \\
\hline NOS1 & 0.00 & 0.00 & 1.00 & 2.00 & 0.510 & 0.556 \\
\hline NRAM & 0.00 & 0.00 & 0.00 & 0.00 & 0.738 & 0.638 \\
\hline PA2GA & 5.05 & 5.05 & 1.01 & 1.01 & 0.570 & 0.507 \\
\hline PARP1 & 5.91 & 10.83 & 5.31 & 7.28 & 0.759 & 0.800 \\
\hline PDE5A & 2.51 & 17.59 & 1.26 & 9.55 & 0.604 & 0.703 \\
\hline PGH1 & 2.56 & 5.13 & 0.51 & 1.54 & 0.625 & 0.638 \\
\hline PGH2 & 0.00 & 2.30 & 1.15 & 2.30 & 0.636 & 0.700 \\
\hline PLK1 & 9.35 & 23.36 & 3.74 & 6.54 & 0.535 & 0.666 \\
\hline PNPH & 0.00 & 0.00 & 2.91 & 7.77 & 0.517 & 0.700 \\
\hline PPARA & 12.06 & 21.45 & 7.77 & 10.99 & 0.714 & 0.781 \\
\hline PPARD & 4.17 & 12.50 & 2.08 & 7.08 & 0.551 & 0.666 \\
\hline PPARG & 9.30 & 14.46 & 5.37 & 7.02 & 0.645 & 0.654 \\
\hline PRGR & 1.71 & 10.24 & 3.07 & 4.44 & 0.675 & 0.726 \\
\hline PTN1 & 23.08 & 38.46 & 13.08 & 18.46 & 0.773 & 0.831 \\
\hline PUR2 & 0.00 & 20.00 & 2.00 & 14.00 & 0.826 & 0.919 \\
\hline PYGM & 0.00 & 0.00 & 1.30 & 3.90 & 0.607 & 0.595 \\
\hline PYRD & 9.01 & 9.01 & 8.11 & 9.01 & 0.778 & 0.813 \\
\hline RENI & 0.00 & 0.00 & 1.92 & 3.85 & 0.691 & 0.694 \\
\hline ROCK1 & 0.00 & 0.00 & 1.00 & 2.00 & 0.603 & 0.705 \\
\hline RXRA & 3.82 & 0.00 & 10.69 & 3.05 & 0.848 & 0.768 \\
\hline SAHH & 0.00 & 7.94 & 0.00 & 3.17 & 0.506 & 0.747 \\
\hline SRC & 3.82 & 20.99 & 2.48 & 10.11 & 0.542 & 0.683 \\
\hline TGFR1 & 0.00 & 7.52 & 5.26 & 15.04 & 0.741 & 0.796 \\
\hline THB & 0.00 & 4.85 & 0.00 & 1.94 & 0.549 & 0.529 \\
\hline THRB & 19.52 & 22.78 & 8.03 & 9.33 & 0.751 & 0.771 \\
\hline TRY1 & 20.04 & 20.04 & 12.69 & 13.14 & 0.798 & 0.806 \\
\hline TRYB1 & 20.27 & 10.14 & 8.11 & 14.19 & 0.726 & 0.740 \\
\hline TYSY & 0.00 & 18.35 & 3.67 & 10.09 & 0.677 & 0.735 \\
\hline UROK & 12.35 & 12.35 & 6.79 & 11.73 & 0.767 & 0.782 \\
\hline VGFR2 & 3.67 & 36.67 & 4.65 & 18.34 & 0.638 & 0.783 \\
\hline WEE1 & 9.80 & 53.92 & 13.73 & 39.22 & 0.849 & 0.877 \\
\hline XIAP & 10.00 & 20.00 & 11.00 & 12.00 & 0.810 & 0.701 \\
\hline
\end{tabular}


Table S15. Outcomes of the screening power test for GoldScore and GoldScore ${ }^{\text {DEWED }}$ (calibrated on the PDBbind core set) on all 102 targets in the DUD-E benchmark

\begin{tabular}{|c|c|c|c|c|c|c|}
\hline \multirow[t]{2}{*}{ Target } & \multicolumn{2}{|c|}{$\mathbf{E F}_{\mathbf{0 . 2} \%}$} & \multicolumn{2}{|c|}{$\mathbf{E F}_{1 \%}$} & \multicolumn{2}{|c|}{ AUC } \\
\hline & GoldScore & $\begin{array}{c}\text { Gold } \\
\text { Score }^{\text {DEWED-G }}\end{array}$ & GoldScore & $\begin{array}{c}\text { Gold } \\
\text { Score }^{\text {DEWED-G }}\end{array}$ & GoldScore & $\begin{array}{c}\text { Gold } \\
\text { Score }^{\text {DEWED-G }}\end{array}$ \\
\hline AA2AR & 7.26 & 6.22 & 3.94 & 4.56 & 0.715 & 0.686 \\
\hline $\mathrm{ABL}$ & 27.47 & 32.97 & 7.69 & 9.89 & 0.755 & 0.809 \\
\hline ACE & 21.28 & 19.50 & 12.41 & 8.51 & 0.491 & 0.609 \\
\hline ACES & 1.10 & 24.28 & 1.32 & 9.27 & 0.509 & 0.620 \\
\hline ADA & 10.75 & 16.13 & 10.75 & 11.83 & 0.661 & 0.635 \\
\hline ADA17 & 2.82 & 11.28 & 3.76 & 8.27 & 0.515 & 0.624 \\
\hline ADRB1 & 0.00 & 2.02 & 0.40 & 2.43 & 0.517 & 0.628 \\
\hline ADRB2 & 0.00 & 8.66 & 0.00 & 3.90 & 0.538 & 0.633 \\
\hline AKT1 & 0.00 & 1.71 & 1.02 & 2.39 & 0.599 & 0.633 \\
\hline AKT2 & 47.01 & 59.83 & 17.09 & 30.77 & 0.746 & 0.798 \\
\hline ALDR & 25.16 & 15.72 & 10.69 & 6.29 & 0.671 & 0.610 \\
\hline AMPC & 10.42 & 0.00 & 6.25 & 0.00 & 0.632 & 0.553 \\
\hline ANDR & 0.00 & 0.00 & 0.00 & 0.37 & 0.584 & 0.553 \\
\hline AOFB & 12.30 & 16.39 & 4.92 & 4.10 & 0.745 & 0.727 \\
\hline BACE1 & 10.79 & 5.40 & 5.40 & 4.68 & 0.574 & 0.551 \\
\hline BRAF & 0.00 & 3.29 & 2.63 & 3.29 & 0.640 & 0.674 \\
\hline CAH2 & 6.11 & 2.04 & 6.52 & 2.24 & 0.629 & 0.599 \\
\hline CASP3 & 30.15 & 52.76 & 14.57 & 16.58 & 0.740 & 0.689 \\
\hline CDK2 & 8.44 & 9.49 & 8.23 & 5.06 & 0.740 & 0.715 \\
\hline COMT & 0.00 & 0.00 & 0.00 & 0.00 & 0.718 & 0.597 \\
\hline CP2C9 & 0.00 & 12.50 & 1.67 & 3.33 & 0.514 & 0.569 \\
\hline CP3A4 & 0.00 & 0.00 & 0.00 & 1.76 & 0.552 & 0.531 \\
\hline CSF1R & 12.05 & 15.06 & 6.63 & 5.42 & 0.522 & 0.560 \\
\hline CXCR4 & 0.00 & 0.00 & 0.00 & 2.50 & 0.511 & 0.552 \\
\hline DEF & 0.00 & 0.00 & 0.00 & 1.96 & 0.596 & 0.532 \\
\hline DHI1 & 0.00 & 0.00 & 0.00 & 0.00 & 0.569 & 0.562 \\
\hline DPP4 & 8.44 & 4.69 & 4.88 & 3.00 & 0.502 & 0.565 \\
\hline DRD3 & 0.00 & 0.00 & 0.00 & 0.42 & 0.546 & 0.528 \\
\hline DYR & 51.95 & 19.48 & 16.45 & 7.36 & 0.649 & 0.752 \\
\hline EGFR & 20.30 & 48.89 & 9.23 & 19.74 & 0.716 & 0.747 \\
\hline ESR1 & 15.71 & 24.87 & 8.90 & 11.26 & 0.603 & 0.634 \\
\hline ESR2 & 2.73 & 10.93 & 1.91 & 6.56 & 0.585 & 0.608 \\
\hline FA10 & 22.35 & 29.80 & 12.10 & 16.76 & 0.560 & 0.720 \\
\hline FA7 & 35.09 & 30.70 & 19.30 & 19.30 & 0.756 & 0.822 \\
\hline FABP4 & 31.91 & 42.55 & 14.89 & 14.89 & 0.738 & 0.681 \\
\hline FAK1 & 15.00 & 45.00 & 11.00 & 17.00 & 0.684 & 0.813 \\
\hline FGFR1 & 0.00 & 0.00 & 0.00 & 1.44 & 0.602 & 0.684 \\
\hline FKB1A & 9.01 & 0.00 & 4.50 & 5.41 & 0.514 & 0.635 \\
\hline FNTA & 4.22 & 9.29 & 3.21 & 4.56 & 0.540 & 0.574 \\
\hline FPPS & 35.29 & 11.76 & 12.94 & 3.53 & 0.695 & 0.580 \\
\hline GCR & 7.75 & 7.75 & 1.94 & 2.71 & 0.709 & 0.585 \\
\hline GLCM & 0.00 & 0.00 & 0.00 & 0.00 & 0.508 & 0.518 \\
\hline GRIA2 & 0.00 & 6.33 & 4.43 & 2.53 & 0.673 & 0.679 \\
\hline GRIK1 & 24.75 & 0.00 & 15.84 & 0.99 & 0.716 & 0.708 \\
\hline HDAC2 & 5.41 & 10.81 & 4.86 & 5.95 & 0.720 & 0.742 \\
\hline HDAC8 & 0.00 & 2.94 & 1.18 & 2.35 & 0.575 & 0.606 \\
\hline HIVINT & 0.00 & 5.00 & 2.00 & 2.00 & 0.586 & 0.610 \\
\hline HIVPR & 0.00 & 2.80 & 0.00 & 1.87 & 0.812 & 0.578 \\
\hline
\end{tabular}




\begin{tabular}{|c|c|c|c|c|c|c|}
\hline HIVRT & 11.83 & 16.27 & 7.40 & 7.10 & 0.683 & 0.679 \\
\hline HMDH & 2.94 & 8.82 & 4.71 & 10.59 & 0.504 & 0.592 \\
\hline HS90A & 17.05 & 5.68 & 4.55 & 2.27 & 0.622 & 0.541 \\
\hline HXK4 & 0.00 & 5.43 & 9.78 & 8.70 & 0.724 & 0.782 \\
\hline IGF1R & 16.89 & 40.54 & 8.78 & 15.54 & 0.531 & 0.593 \\
\hline INHA & 11.63 & 11.63 & 9.30 & 6.98 & 0.709 & 0.733 \\
\hline ITAL & 0.00 & 0.00 & 2.17 & 0.72 & 0.506 & 0.534 \\
\hline JAK2 & 23.36 & 51.40 & 9.35 & 14.02 & 0.752 & 0.764 \\
\hline KIF11 & 8.62 & 8.62 & 5.17 & 10.34 & 0.661 & 0.724 \\
\hline KIT & 6.02 & 3.01 & 2.41 & 3.01 & 0.517 & 0.530 \\
\hline KITH & 17.54 & 17.54 & 22.81 & 26.32 & 0.726 & 0.745 \\
\hline KPCB & 14.81 & 22.22 & 12.59 & 11.11 & 0.657 & 0.705 \\
\hline LCK & 7.14 & 11.90 & 4.52 & 6.19 & 0.568 & 0.658 \\
\hline LKHA4 & 8.77 & 20.47 & 8.19 & 10.53 & 0.693 & 0.786 \\
\hline MAPK2 & 0.00 & 4.95 & 13.86 & 10.89 & 0.797 & 0.815 \\
\hline MCR & 0.00 & 0.00 & 0.00 & 0.00 & 0.683 & 0.610 \\
\hline MET & 27.11 & 60.24 & 15.66 & 33.73 & 0.840 & 0.879 \\
\hline MK01 & 0.00 & 0.00 & 3.80 & 0.00 & 0.702 & 0.742 \\
\hline MK10 & 9.62 & 4.81 & 3.85 & 9.62 & 0.665 & 0.681 \\
\hline MK14 & 0.87 & 3.46 & 2.25 & 3.11 & 0.632 & 0.648 \\
\hline MMP13 & 12.24 & 13.11 & 7.52 & 6.82 & 0.581 & 0.619 \\
\hline MP2K1 & 4.13 & 0.00 & 3.31 & 1.65 & 0.587 & 0.616 \\
\hline NOS1 & 35.00 & 20.00 & 14.00 & 12.00 & 0.707 & 0.634 \\
\hline NRAM & 0.00 & 0.00 & 0.00 & 0.00 & 0.577 & 0.619 \\
\hline PA2GA & 0.00 & 0.00 & 4.04 & 4.04 & 0.547 & 0.561 \\
\hline PARP1 & 10.83 & 4.92 & 8.66 & 3.54 & 0.631 & 0.736 \\
\hline PDE5A & 13.82 & 18.84 & 6.53 & 9.55 & 0.705 & 0.695 \\
\hline PGH1 & 12.82 & 15.38 & 9.23 & 8.72 & 0.598 & 0.612 \\
\hline PGH2 & 24.14 & 14.94 & 18.62 & 18.16 & 0.715 & 0.728 \\
\hline PLK1 & 0.00 & 18.69 & 0.00 & 4.67 & 0.563 & 0.695 \\
\hline PNPH & 67.96 & 38.83 & 44.66 & 19.42 & 0.868 & 0.767 \\
\hline PPARA & 6.70 & 8.04 & 3.75 & 5.90 & 0.563 & 0.690 \\
\hline PPARD & 2.08 & 4.17 & 2.08 & 3.75 & 0.600 & 0.656 \\
\hline PPARG & 2.07 & 6.20 & 1.45 & 4.34 & 0.547 & 0.655 \\
\hline PRGR & 0.00 & 0.00 & 0.34 & 0.68 & 0.535 & 0.510 \\
\hline PTN1 & 57.69 & 57.69 & 19.23 & 22.31 & 0.664 & 0.712 \\
\hline PUR2 & 50.00 & 50.00 & 36.00 & 44.00 & 0.828 & 0.937 \\
\hline PYGM & 0.00 & 0.00 & 0.00 & 0.00 & 0.543 & 0.551 \\
\hline PYRD & 49.55 & 9.01 & 20.72 & 8.11 & 0.618 & 0.582 \\
\hline RENI & 0.00 & 4.81 & 0.00 & 2.88 & 0.610 & 0.517 \\
\hline ROCK1 & 0.00 & 0.00 & 3.00 & 1.00 & 0.647 & 0.657 \\
\hline RXRA & 0.00 & 0.00 & 0.00 & 2.29 & 0.540 & 0.575 \\
\hline SAHH & 47.62 & 23.81 & 36.51 & 12.70 & 0.908 & 0.904 \\
\hline SRC & 0.95 & 3.82 & 0.57 & 4.01 & 0.602 & 0.646 \\
\hline TGFR1 & 30.08 & 41.35 & 18.05 & 17.29 & 0.827 & 0.851 \\
\hline THB & 19.42 & 14.56 & 10.68 & 7.77 & 0.772 & 0.791 \\
\hline THRB & 14.10 & 21.69 & 7.38 & 11.93 & 0.622 & 0.764 \\
\hline TRY1 & 8.91 & 12.25 & 8.91 & 9.13 & 0.634 & 0.748 \\
\hline TRYB1 & 3.38 & 6.76 & 1.35 & 4.73 & 0.564 & 0.638 \\
\hline TYSY & 45.87 & 45.87 & 18.35 & 16.51 & 0.743 & 0.789 \\
\hline UROK & 18.52 & 18.52 & 13.58 & 11.11 & 0.726 & 0.780 \\
\hline VGFR2 & 6.11 & 9.78 & 3.67 & 5.13 & 0.650 & 0.705 \\
\hline WEE1 & 58.82 & 58.82 & 56.86 & 54.90 & 0.974 & 0.958 \\
\hline XIAP & 35.00 & 45.00 & 16.00 & 23.00 & 0.729 & 0.780 \\
\hline
\end{tabular}


Table S16. Outcomes of the screening power test for ASP and ASP DEWED (calibrated on the PDBbind core set) on all 102 targets in the DUD-E benchmark

\begin{tabular}{|c|c|c|c|c|c|c|}
\hline \multirow[t]{2}{*}{ Target } & \multicolumn{2}{|c|}{$\mathbf{E F}_{\mathbf{0 . 2} \%}$} & \multicolumn{2}{|c|}{$\mathbf{E F}_{1 \%}$} & \multicolumn{2}{|c|}{ AUC } \\
\hline & ASP & ASPDEWED-G & ASP & ASPDEWED-G & ASP & ASP $^{\text {DEWED-G }}$ \\
\hline AA2AR & 31.12 & 25.93 & 15.98 & 10.79 & 0.855 & 0.787 \\
\hline $\mathrm{ABL}$ & 43.96 & 43.96 & 24.73 & 26.37 & 0.867 & 0.852 \\
\hline $\mathrm{ACE}$ & 10.64 & 12.41 & 4.96 & 6.38 & 0.585 & 0.590 \\
\hline ACES & 41.94 & 49.67 & 21.41 & 20.31 & 0.753 & 0.769 \\
\hline ADA & 32.26 & 32.26 & 12.90 & 13.98 & 0.796 & 0.744 \\
\hline ADA17 & 15.04 & 15.04 & 6.39 & 8.46 & 0.536 & 0.594 \\
\hline ADRB1 & 4.86 & 4.05 & 4.05 & 5.26 & 0.753 & 0.732 \\
\hline ADRB2 & 2.16 & 4.33 & 3.90 & 6.06 & 0.759 & 0.742 \\
\hline AKT1 & 22.18 & 22.18 & 14.68 & 9.56 & 0.811 & 0.776 \\
\hline AKT2 & 17.09 & 38.46 & 19.66 & 24.79 & 0.839 & 0.806 \\
\hline ALDR & 6.29 & 3.14 & 6.92 & 3.77 & 0.691 & 0.655 \\
\hline AMPC & 0.00 & 0.00 & 0.00 & 0.00 & 0.520 & 0.568 \\
\hline ANDR & 3.72 & 1.86 & 4.83 & 5.20 & 0.578 & 0.567 \\
\hline AOFB & 8.20 & 12.30 & 6.56 & 5.74 & 0.720 & 0.704 \\
\hline BACE1 & 12.59 & 19.78 & 10.43 & 14.39 & 0.740 & 0.750 \\
\hline BRAF & 23.03 & 29.61 & 12.50 & 10.53 & 0.823 & 0.778 \\
\hline CAH2 & 4.07 & 4.07 & 2.44 & 3.05 & 0.599 & 0.578 \\
\hline CASP3 & 27.64 & 30.15 & 13.57 & 15.08 & 0.708 & 0.684 \\
\hline CDK2 & 17.93 & 11.60 & 10.55 & 6.54 & 0.778 & 0.715 \\
\hline COMT & 0.00 & 0.00 & 2.44 & 0.00 & 0.536 & 0.496 \\
\hline СР2C9 & 4.17 & 0.00 & 0.83 & 0.83 & 0.678 & 0.646 \\
\hline CP3A4 & 17.65 & 23.53 & 12.35 & 12.35 & 0.677 & 0.657 \\
\hline CSF1R & 24.10 & 24.10 & 9.04 & 7.23 & 0.693 & 0.682 \\
\hline CXCR4 & 12.50 & 12.50 & 7.50 & 7.50 & 0.676 & 0.703 \\
\hline DEF & 0.00 & 0.00 & 0.00 & 0.00 & 0.613 & 0.480 \\
\hline DHI1 & 1.52 & 3.03 & 0.61 & 0.61 & 0.581 & 0.608 \\
\hline DPP4 & 12.20 & 9.38 & 5.25 & 4.88 & 0.657 & 0.661 \\
\hline DRD3 & 4.17 & 4.17 & 3.54 & 4.58 & 0.684 & 0.668 \\
\hline DYR & 73.59 & 67.10 & 49.35 & 34.63 & 0.933 & 0.905 \\
\hline EGFR & 56.27 & 54.43 & 20.66 & 17.90 & 0.798 & 0.746 \\
\hline ESR1 & 30.10 & 30.10 & 18.06 & 14.92 & 0.780 & 0.729 \\
\hline ESR2 & 31.42 & 27.32 & 18.58 & 16.39 & 0.794 & 0.733 \\
\hline FA10 & 48.42 & 47.49 & 26.07 & 28.12 & 0.753 & 0.741 \\
\hline FA7 & 57.02 & 57.02 & 41.23 & 40.35 & 0.875 & 0.872 \\
\hline FABP4 & 0.00 & 0.00 & 12.77 & 12.77 & 0.848 & 0.755 \\
\hline FAK1 & 20.00 & 30.00 & 14.00 & 17.00 & 0.885 & 0.864 \\
\hline FGFR1 & 10.79 & 10.79 & 8.63 & 9.35 & 0.808 & 0.783 \\
\hline FKB1A & 0.00 & 0.00 & 1.80 & 2.70 & 0.534 & 0.543 \\
\hline FNTA & 4.22 & 5.91 & 4.73 & 4.73 & 0.715 & 0.707 \\
\hline FPPS & 88.24 & 29.41 & 44.71 & 17.65 & 0.885 & 0.767 \\
\hline GCR & 17.44 & 17.44 & 12.02 & 10.47 & 0.579 & 0.556 \\
\hline GLCM & 27.78 & 27.78 & 14.81 & 11.11 & 0.621 & 0.589 \\
\hline GRIA2 & 34.81 & 18.99 & 24.68 & 10.13 & 0.783 & 0.755 \\
\hline GRIK1 & 24.75 & 9.90 & 9.90 & 5.94 & 0.837 & 0.776 \\
\hline HDAC2 & 21.62 & 21.62 & 10.27 & 9.19 & 0.767 & 0.772 \\
\hline HDAC8 & 0.00 & 0.00 & 2.94 & 2.35 & 0.690 & 0.669 \\
\hline HIVINT & 25.00 & 15.00 & 13.00 & 10.00 & 0.805 & 0.733 \\
\hline HIVPR & 6.53 & 23.32 & 3.92 & 8.96 & 0.550 & 0.609 \\
\hline HIVRT & 7.40 & 10.36 & 6.21 & 6.80 & 0.728 & 0.741 \\
\hline
\end{tabular}




\begin{tabular}{|c|c|c|c|c|c|c|}
\hline HMDH & 11.76 & 26.47 & 12.35 & 20.59 & 0.788 & 0.823 \\
\hline HS90A & 5.68 & 5.68 & 1.14 & 1.14 & 0.593 & 0.535 \\
\hline HXK4 & 21.74 & 10.87 & 10.87 & 9.78 & 0.767 & 0.684 \\
\hline IGF1R & 33.78 & 27.03 & 14.19 & 16.22 & 0.766 & 0.763 \\
\hline INHA & 0.00 & 0.00 & 4.65 & 4.65 & 0.790 & 0.765 \\
\hline ITAL & 0.00 & 0.00 & 0.72 & 1.45 & 0.535 & 0.466 \\
\hline JAK2 & 51.40 & 56.07 & 23.36 & 20.56 & 0.822 & 0.797 \\
\hline KIF11 & 25.86 & 34.48 & 26.72 & 28.45 & 0.846 & 0.823 \\
\hline KIT & 9.04 & 6.02 & 7.83 & 6.02 & 0.742 & 0.739 \\
\hline KITH & 52.63 & 52.63 & 43.86 & 43.86 & 0.851 & 0.834 \\
\hline KPCB & 29.63 & 25.93 & 22.22 & 17.04 & 0.749 & 0.735 \\
\hline LCK & 22.62 & 23.81 & 12.38 & 12.14 & 0.787 & 0.747 \\
\hline LKHA4 & 29.24 & 32.16 & 21.05 & 19.88 & 0.881 & 0.873 \\
\hline MAPK2 & 34.65 & 14.85 & 26.73 & 13.86 & 0.906 & 0.863 \\
\hline MCR & 0.00 & 0.00 & 1.06 & 1.06 & 0.515 & 0.482 \\
\hline MET & 69.28 & 69.28 & 54.22 & 52.41 & 0.919 & 0.902 \\
\hline MK01 & 0.00 & 0.00 & 1.27 & 1.27 & 0.792 & 0.774 \\
\hline MK10 & 19.23 & 14.42 & 9.62 & 7.69 & 0.804 & 0.766 \\
\hline MK14 & 15.57 & 24.22 & 10.21 & 12.28 & 0.746 & 0.698 \\
\hline MMP13 & 15.73 & 13.11 & 9.79 & 8.74 & 0.700 & 0.659 \\
\hline MP2K1 & 0.00 & 0.00 & 0.00 & 0.00 & 0.709 & 0.676 \\
\hline NOS1 & 25.00 & 25.00 & 15.00 & 10.00 & 0.715 & 0.675 \\
\hline NRAM & 0.00 & 0.00 & 3.06 & 1.02 & 0.845 & 0.839 \\
\hline PA2GA & 5.05 & 5.05 & 5.05 & 6.06 & 0.737 & 0.669 \\
\hline PARP1 & 26.57 & 3.94 & 18.11 & 8.66 & 0.889 & 0.843 \\
\hline PDE5A & 31.41 & 30.15 & 22.86 & 16.08 & 0.884 & 0.851 \\
\hline PGH1 & 2.56 & 2.56 & 1.54 & 0.51 & 0.566 & 0.558 \\
\hline PGH2 & 12.64 & 12.64 & 11.49 & 12.87 & 0.724 & 0.726 \\
\hline PLK1 & 37.38 & 37.38 & 16.82 & 13.08 & 0.749 & 0.719 \\
\hline PNPH & 53.40 & 43.69 & 30.10 & 18.45 & 0.901 & 0.847 \\
\hline PPARA & 13.40 & 13.40 & 16.62 & 16.09 & 0.872 & 0.879 \\
\hline PPARD & 10.42 & 14.58 & 7.92 & 9.58 & 0.799 & 0.815 \\
\hline PPARG & 10.33 & 12.40 & 7.23 & 8.47 & 0.804 & 0.811 \\
\hline PRGR & 3.41 & 5.12 & 4.44 & 5.80 & 0.666 & 0.655 \\
\hline PTN1 & 30.77 & 23.08 & 16.92 & 15.38 & 0.799 & 0.772 \\
\hline PUR2 & 50.00 & 50.00 & 54.00 & 54.00 & 0.999 & 0.998 \\
\hline PYGM & 6.49 & 0.00 & 2.60 & 3.90 & 0.553 & 0.559 \\
\hline PYRD & 9.01 & 9.01 & 10.81 & 6.31 & 0.727 & 0.691 \\
\hline RENI & 24.04 & 28.85 & 8.65 & 11.54 & 0.622 & 0.711 \\
\hline ROCK1 & 0.00 & 0.00 & 1.00 & 1.00 & 0.780 & 0.732 \\
\hline RXRA & 0.00 & 7.63 & 0.76 & 5.34 & 0.643 & 0.714 \\
\hline SAHH & 47.62 & 39.68 & 42.86 & 17.46 & 0.979 & 0.953 \\
\hline SRC & 0.95 & 2.86 & 1.91 & 3.82 & 0.667 & 0.674 \\
\hline TGFR1 & 37.59 & 33.83 & 30.83 & 25.56 & 0.935 & 0.909 \\
\hline THB & 19.42 & 14.56 & 11.65 & 10.68 & 0.765 & 0.779 \\
\hline THRB & 56.40 & 56.40 & 26.25 & 26.03 & 0.832 & 0.839 \\
\hline TRY1 & 57.91 & 55.68 & 41.43 & 40.53 & 0.839 & 0.844 \\
\hline TRYB1 & 54.05 & 43.92 & 18.92 & 18.92 & 0.734 & 0.749 \\
\hline TYSY & 41.28 & 32.11 & 25.69 & 19.27 & 0.860 & 0.844 \\
\hline UROK & 61.73 & 61.73 & 55.56 & 46.30 & 0.896 & 0.887 \\
\hline VGFR2 & 33.01 & 30.56 & 14.67 & 14.18 & 0.791 & 0.776 \\
\hline WEE1 & 58.82 & 58.82 & 58.82 & 55.88 & 0.989 & 0.970 \\
\hline XIAP & 10.00 & 15.00 & 5.00 & 15.00 & 0.706 & 0.715 \\
\hline
\end{tabular}


Table S17. Outcomes of the screening power test for ChemPLP and ChemPLP ${ }^{\text {DEWED }}$ (calibrated on the PDBbind core set) on all 102 targets in the DUD-E benchmark

\begin{tabular}{|c|c|c|c|c|c|c|}
\hline \multirow[t]{2}{*}{ Target } & \multicolumn{2}{|c|}{$\mathbf{E F}_{\mathbf{0 . 2} \%}$} & \multicolumn{2}{|c|}{$\mathbf{E F}_{1 \%}$} & \multicolumn{2}{|c|}{ AUC } \\
\hline & ChemPLP & $\underset{\text { EWED-G }}{\text { ChemPLPD }}$ & ChemPLP & $\underset{\text { EWED-G }}{\text { ChemPLPD }}$ & ChemPLP & $\underset{\text { EWED-G }}{\text { ChemPLP }^{\mathrm{D}}}$ \\
\hline AA2AR & 8.30 & 9.34 & 3.53 & 4.36 & 0.705 & 0.637 \\
\hline$\overline{\mathrm{ABL}}$ & 30.22 & 5.49 & 10.99 & 4.95 & 0.755 & 0.766 \\
\hline ACE & 30.14 & 3.55 & 15.96 & 5.32 & 0.719 & 0.709 \\
\hline ACES & 43.05 & 39.74 & 19.87 & 14.35 & 0.726 & 0.750 \\
\hline ADA & 37.63 & 16.13 & 12.90 & 9.68 & 0.725 & 0.657 \\
\hline ADA17 & 39.47 & 16.92 & 21.24 & 10.90 & 0.693 & 0.657 \\
\hline ADRB1 & 14.17 & 10.12 & 10.12 & 6.48 & 0.781 & 0.682 \\
\hline ADRB2 & 12.99 & 10.82 & 7.36 & 5.63 & 0.772 & 0.674 \\
\hline AKT1 & 8.53 & 3.41 & 8.87 & 3.75 & 0.693 & 0.700 \\
\hline AKT2 & 34.19 & 21.37 & 10.26 & 16.24 & 0.771 & 0.714 \\
\hline ALDR & 15.72 & 0.00 & 11.32 & 2.52 & 0.642 & 0.612 \\
\hline AMPC & 0.00 & 0.00 & 0.00 & 0.00 & 0.547 & 0.505 \\
\hline ANDR & 3.72 & 0.00 & 4.09 & 0.00 & 0.518 & 0.518 \\
\hline AOFB & 4.10 & 0.00 & 4.92 & 2.46 & 0.736 & 0.653 \\
\hline BACE1 & 12.59 & 16.19 & 7.55 & 10.07 & 0.705 & 0.709 \\
\hline BRAF & 3.29 & 3.29 & 5.92 & 3.29 & 0.713 & 0.731 \\
\hline CAH2 & 3.05 & 1.02 & 1.43 & 0.81 & 0.522 & 0.548 \\
\hline CASP3 & 52.76 & 40.20 & 25.13 & 16.58 & 0.735 & 0.675 \\
\hline CDK2 & 4.22 & 1.05 & 5.70 & 1.69 & 0.694 & 0.608 \\
\hline COMT & 0.00 & 0.00 & 0.00 & 0.00 & 0.647 & 0.527 \\
\hline CP2C9 & 4.17 & 4.17 & 0.83 & 5.83 & 0.574 & 0.555 \\
\hline CP3A4 & 5.88 & 5.88 & 2.35 & 5.29 & 0.563 & 0.556 \\
\hline CSF1R & 9.04 & 3.01 & 3.61 & 4.22 & 0.610 & 0.663 \\
\hline CXCR4 & 0.00 & 0.00 & 0.00 & 0.00 & 0.636 & 0.682 \\
\hline DEF & 0.00 & 4.90 & 4.90 & 0.98 & 0.700 & 0.648 \\
\hline DHI1 & 0.00 & 0.00 & 0.91 & 0.61 & 0.525 & 0.527 \\
\hline DPP4 & 15.01 & 0.00 & 8.26 & 1.88 & 0.732 & 0.700 \\
\hline DRD3 & 1.04 & 3.13 & 1.67 & 4.17 & 0.686 & 0.632 \\
\hline DYR & 8.66 & 8.66 & 14.72 & 4.33 & 0.842 & 0.742 \\
\hline EGFR & 54.43 & 35.06 & 19.00 & 16.05 & 0.730 & 0.701 \\
\hline ESR1 & 40.58 & 13.09 & 19.90 & 9.42 & 0.750 & 0.712 \\
\hline ESR2 & 28.69 & 16.39 & 15.57 & 6.28 & 0.723 & 0.666 \\
\hline FA10 & 31.66 & 20.48 & 17.69 & 13.97 & 0.719 & 0.738 \\
\hline FA7 & 43.86 & 26.32 & 30.70 & 15.79 & 0.813 & 0.819 \\
\hline FABP4 & 53.19 & 10.64 & 23.40 & 4.26 & 0.846 & 0.629 \\
\hline FAK1 & 35.00 & 20.00 & 25.00 & 10.00 & 0.825 & 0.842 \\
\hline FGFR1 & 3.60 & 0.00 & 3.60 & 0.72 & 0.715 & 0.719 \\
\hline FKB1A & 0.00 & 0.00 & 0.00 & 0.00 & 0.513 & 0.614 \\
\hline FNTA & 5.07 & 9.29 & 3.55 & 3.72 & 0.639 & 0.600 \\
\hline FPPS & 70.59 & 0.00 & 49.41 & 0.00 & 0.975 & 0.652 \\
\hline GCR & 1.94 & 1.94 & 1.16 & 2.71 & 0.567 & 0.528 \\
\hline GLCM & 64.81 & 9.26 & 14.81 & 7.41 & 0.701 & 0.609 \\
\hline GRIA2 & 18.99 & 9.49 & 11.39 & 8.23 & 0.770 & 0.631 \\
\hline GRIK1 & 19.80 & 0.00 & 10.89 & 0.99 & 0.850 & 0.616 \\
\hline HDAC2 & 35.14 & 8.11 & 14.05 & 8.65 & 0.851 & 0.769 \\
\hline HDAC8 & 5.88 & 5.88 & 7.06 & 1.18 & 0.727 & 0.694 \\
\hline HIVINT & 10.00 & 5.00 & 9.00 & 5.00 & 0.756 & 0.605 \\
\hline HIVPR & 3.73 & 9.33 & 2.99 & 5.41 & 0.521 & 0.607 \\
\hline
\end{tabular}




\begin{tabular}{|c|c|c|c|c|c|c|}
\hline HIVRT & 11.83 & 5.92 & 7.10 & 3.85 & 0.679 & 0.675 \\
\hline HMDH & 38.24 & 14.71 & 19.41 & 10.00 & 0.817 & 0.746 \\
\hline HS90A & 5.68 & 5.68 & 2.27 & 1.14 & 0.554 & 0.532 \\
\hline HXK4 & 27.17 & 0.00 & 8.70 & 0.00 & 0.689 & 0.688 \\
\hline IGF1R & 54.05 & 20.27 & 16.22 & 11.49 & 0.723 & 0.753 \\
\hline INHA & 11.63 & 11.63 & 6.98 & 2.33 & 0.768 & 0.768 \\
\hline ITAL & 0.00 & 0.00 & 0.00 & 0.00 & 0.540 & 0.593 \\
\hline JAK2 & 37.38 & 42.06 & 16.82 & 14.95 & 0.804 & 0.740 \\
\hline KIF11 & 38.79 & 4.31 & 29.31 & 13.79 & 0.791 & 0.806 \\
\hline KIT & 9.04 & 18.07 & 4.82 & 6.63 & 0.551 & 0.588 \\
\hline KITH & 43.86 & 0.00 & 21.05 & 1.75 & 0.764 & 0.760 \\
\hline KPCB & 59.26 & 14.81 & 26.67 & 8.15 & 0.808 & 0.766 \\
\hline LCK & 8.33 & 4.76 & 8.57 & 4.05 & 0.676 & 0.669 \\
\hline LKHA4 & 20.47 & 23.39 & 18.71 & 12.87 & 0.886 & 0.882 \\
\hline MAPK2 & 19.80 & 4.95 & 21.78 & 5.94 & 0.878 & 0.864 \\
\hline MCR & 0.00 & 0.00 & 2.13 & 0.00 & 0.627 & 0.615 \\
\hline MET & 63.25 & 45.18 & 39.16 & 22.29 & 0.838 & 0.834 \\
\hline MK01 & 0.00 & 0.00 & 1.27 & 2.53 & 0.770 & 0.757 \\
\hline MK10 & 14.42 & 4.81 & 9.62 & 4.81 & 0.707 & 0.695 \\
\hline MK14 & 13.84 & 21.63 & 7.61 & 9.86 & 0.658 & 0.675 \\
\hline MMP13 & 39.34 & 7.87 & 15.91 & 5.94 & 0.656 & 0.606 \\
\hline MP2K1 & 0.00 & 4.13 & 2.48 & 2.48 & 0.691 & 0.642 \\
\hline NOS1 & 10.00 & 5.00 & 8.00 & 7.00 & 0.730 & 0.657 \\
\hline NRAM & 5.10 & 0.00 & 3.06 & 2.04 & 0.829 & 0.768 \\
\hline PA2GA & 0.00 & 0.00 & 8.08 & 7.07 & 0.728 & 0.669 \\
\hline PARP1 & 37.40 & 0.98 & 22.83 & 2.76 & 0.870 & 0.843 \\
\hline PDE5A & 18.84 & 12.56 & 15.08 & 6.03 & 0.822 & 0.803 \\
\hline PGH1 & 0.00 & 0.00 & 1.03 & 1.03 & 0.565 & 0.551 \\
\hline PGH2 & 4.60 & 9.20 & 7.13 & 4.83 & 0.723 & 0.703 \\
\hline PLK1 & 32.71 & 23.36 & 10.28 & 7.48 & 0.772 & 0.740 \\
\hline PNPH & 63.11 & 24.27 & 28.16 & 9.71 & 0.785 & 0.751 \\
\hline PPARA & 17.43 & 22.79 & 14.75 & 12.87 & 0.852 & 0.851 \\
\hline PPARD & 8.33 & 6.25 & 7.08 & 4.58 & 0.810 & 0.780 \\
\hline PPARG & 9.30 & 6.20 & 9.71 & 6.82 & 0.783 & 0.779 \\
\hline PRGR & 3.41 & 0.00 & 4.10 & 0.34 & 0.581 & 0.573 \\
\hline PTN1 & 53.85 & 15.38 & 20.77 & 13.08 & 0.844 & 0.789 \\
\hline PUR2 & 50.00 & 40.00 & 52.00 & 20.00 & 0.993 & 0.985 \\
\hline PYGM & 6.49 & 0.00 & 2.60 & 2.60 & 0.574 & 0.567 \\
\hline PYRD & 40.54 & 4.50 & 24.32 & 1.80 & 0.766 & 0.722 \\
\hline RENI & 28.85 & 28.85 & 10.58 & 13.46 & 0.680 & 0.717 \\
\hline ROCK1 & 5.00 & 0.00 & 1.00 & 0.00 & 0.677 & 0.662 \\
\hline RXRA & 22.90 & 7.63 & 20.61 & 8.40 & 0.807 & 0.753 \\
\hline SAHH & 47.62 & 15.87 & 34.92 & 4.76 & 0.983 & 0.950 \\
\hline SRC & 3.82 & 11.45 & 3.82 & 5.73 & 0.687 & 0.660 \\
\hline TGFR1 & 33.83 & 7.52 & 19.55 & 12.03 & 0.867 & 0.873 \\
\hline THB & 9.71 & 0.00 & 6.80 & 0.00 & 0.756 & 0.711 \\
\hline THRB & 43.38 & 22.78 & 21.48 & 12.80 & 0.828 & 0.827 \\
\hline TRY1 & 21.16 & 10.02 & 14.92 & 6.24 & 0.774 & 0.771 \\
\hline TRYB1 & 30.41 & 16.89 & 12.84 & 8.11 & 0.702 & 0.737 \\
\hline TYSY & 36.70 & 22.94 & 19.27 & 16.51 & 0.833 & 0.809 \\
\hline UROK & 49.38 & 12.35 & 32.10 & 9.26 & 0.797 & 0.788 \\
\hline VGFR2 & 17.11 & 8.56 & 10.51 & 5.38 & 0.711 & 0.731 \\
\hline WEE1 & 58.82 & 44.12 & 57.84 & 38.24 & 0.986 & 0.951 \\
\hline XIAP & 50.00 & 30.00 & 32.00 & 20.00 & 0.832 & 0.795 \\
\hline
\end{tabular}




\section{Reference}

1. Abel, R.; Young, T.; Farid, R.; Berne, B. J.; Friesner, R. A. Role of the active-site solvent in the thermodynamics of factor Xa ligand binding. J. Am. Chem. Soc. 2008, 130, 2817-2831.

2. Li, Y.; Han, L.; Liu, Z.; Wang, R. Comparative assessment of scoring functions on an updated benchmark: 2. Evaluation methods and general results. J. Chem. Inf. Model. 2014, 54, 1717-1736.

3. Lipkowitz, K. B., Boyd, D. B. Genetical gorithms and their use in chemistry. Reviews in Computational Chemistry, Volume10, John Wiley \& Sons Inc. 2007, 10, pp1-73. 